\title{
STAGGERED $t$-STRUCTURES ON DERIVED CATEGORIES OF EQUIVARIANT COHERENT SHEAVES
}

\author{
PRAMOD N. ACHAR
}

\begin{abstract}
Let $X$ be a scheme, and let $G$ be an affine group scheme acting on $X$. Under reasonable hypotheses on $X$ and $G$, we construct a $t$-structure on the derived category of $G$-equivariant coherent sheaves that in many ways resembles the perverse coherent $t$-structure, but which incorporates additional information from the $G$-action. Under certain circumstances, the heart of this $t$-structure, called the "staggered $t$-structure," is a finite-length category, and its simple objects are particularly easy to describe. We also exhibit two small examples in which the staggered $t$-structure is better-behaved than the perverse coherent $t$-structure.
\end{abstract}

\section{INTRODUCTION}

Let $X$ be a scheme (say, over a field), and let $G$ be an affine group scheme acting on $X$. Perverse coherent sheaves are the objects in the heart of a certain nonstandard $t$-structure on the bounded derived category of equivariant coherent sheaves on $X$, first introduced by Deligne, but now more widely known from an exposition by Bezrukavnikov B1. One key feature of this category, which we denote $\mathcal{P}(X)$, is that it interacts well with Grothendieck-Serre duality, just as the much better-known category of perverse (constructible) sheaves interacts well with Poincaré-Verdier duality. Assume now that $G$ acts on $X$ with finitely many orbits. If the boundary of each orbit has codimension at least 2 in the closure of that orbit, then $\mathcal{P}(X)$ also enjoys the following remarkable properties:

- There are "middle-extension functors" that associate to any irreducible equivariant vector bundle on an orbit a certain simple perverse coherent sheaf, supported on the closure of that orbit.

- Every simple perverse coherent sheaf arises in this way.

- $\mathcal{P}(X)$ is a finite-length category; i.e., every object has finite length.

(If we replace "vector bundle" by "local system" and delete the word "coherent" throughout, we get a list of well-known and important properties of perverse sheaves.) A particularly nice situation occurs when all orbits have even codimension: then $\mathcal{P}(X)$ can be made self-dual. A key example of the latter is that in which $G$ is a reductive algebraic group over an algebraically closed field, and $X$ is its unipotent variety (see, for instance, [A1] and [B2]).

However, there are many common examples in representation theory in which the codimension condition does not hold: for instance, the orbits of a reductive algebraic group on the Springer resolution of its unipotent variety, or the orbits of a Borel subgroup on the flag variety. To understand what goes wrong with perverse

The author was partly supported by NSF grant DMS-0500873. 
coherent sheaves on such varieties, let us consider the self-dual case. Roughly speaking, the reason for the even codimension condition is this: the local cohomology of a (suitably normalized) dualizing complex on a given orbit $C$ is concentrated in degree $\operatorname{codim} C$, so in order to define a self-dual $t$-structure, we must be able to put coherent sheaves on $C$ in degree $\frac{1}{2} \operatorname{codim} C$. (For comparison, the dualizing complex for Poincaré-Verdier duality on a topologically stratified space (resp. algebraic variety) measures the real codimension (resp. twice the algebraic codimension) of a given stratum, so in order to have a self-dual category of perverse constructible sheaves, all strata must have even real codimension (resp. there is no restriction on codimensions).)

The main idea of this paper is to use extra information coming from the equivariant structure on each orbit to "stretch out" our interpretation of what the dualizing complex is measuring. Suppose that on each $G$-orbit, each irreducible equivariant vector bundle $\mathcal{L}$ is assigned an integer invariant, called its step, satisfying certain compatibility conditions with tensor products, Hom-sheaves, etc. Now, given $d \in \mathbb{Z}$, consider the shifted object $\mathcal{L}[d]$ in the derived category: this is concentrated in degree $-d$. Let us declare the staggered degree of $\mathcal{L}[d]$ to be $-d+\operatorname{step} \mathcal{L}$. We can then try to refine the construction of the perverse coherent $t$-structure in a way that keeps track of the interaction between Grothendieck-Serre duality and staggered degrees.

We show in this paper that this idea can indeed be carried out: we construct a "staggered $t$-structure" on the bounded derived category of equivariant coherent sheaves. This $t$-structure resembles the perverse coherent $t$-structure in many ways, most significantly in terms of its interaction with Grothendieck-Serre duality. Staggered sheaves on a single $G$-orbit are objects in the derived category with a certain fixed staggered degree, just as perverse coherent sheaves on a single $G$-orbit are objects concentrated in a certain fixed degree in the derived category.

Moreover, we can define the staggered codimension of closed $G$-invariant subschemes in terms of staggered degrees of the dualizing complex. It turns out that if $G$ acts on $X$ with finitely many orbits, each of which has a boundary of staggered codimension at least 2 , then the most desirable properties of perverse sheaves hold: every object in the heart of the staggered $t$-structure has finite length, and every simple object arises by middle-extension of an irreducible vector bundle on a single orbit.

Of course, for this construction to be useful, there must be examples in which the category of staggered sheaves is finite-length but the category of perverse coherent sheaves is not. We exhibit two small examples in which this is the case: that of $\mathbb{C}^{\times}$-equivariant sheaves on $\mathbb{C}$, and that of $B$-equivariant sheaves on $\mathbb{C P}^{1}$, where $B$ is a Borel subgroup of $S L_{2}(\mathbb{C}) .\left(\mathbb{C P}^{1}\right.$ is, of course, the flag variety for $S L_{2}(\mathbb{C})$.) Both of these spaces consist of two orbits, of dimensions 0 and 1 ; the category of perverse coherent sheaves in these cases is not finite-length. However, in both cases, the 0 -dimensional orbit turns out to have staggered codimension at least 2 , so the category of staggered sheaves is finite-length. It is hoped that similar results turn out to be true in broader classes of examples. It would be interesting to compare the staggered $t$-structure to other $t$-structures known to have finite-length hearts, such as the "exotic" $t$-structure for equivariant coherent sheaves on the Springer resolution, described in [B3]. 
For the sake of aesthetics and generality, most of the paper (Sections 28 and part of Section 9) is written without the assumption that $G$ acts with finitely many orbits, although the author is not aware of an interesting example in which that is the case. Section 2 lists notation and collects some basic facts about coherent sheaves and abelian categories. In Section 3 , we axiomatize the additional information that our coherent sheaves should carry with the notion of " $s$-structure." We actually need a global version of this information, not just one instance on each orbit. To that end, Sections 4 and 5 are aimed at proving a "gluing theorem" for $s$-structures. Next, in Section [6] we study the interaction between $s$-structures and Grothendieck-Serre duality. In Sections 7 and 8, we finally define the staggered $t$-structure, and prove that it actually is a $t$-structure. Section 9 gives the construction of the middle-extension functor and a criterion for the heart of the staggered $t$-structure to be finite-length. In Section 10, we prove a theorem that helps in calculating examples by simplifying the checking of the (rather long) list of axioms for an $s$-structure. Finally, Section[1] contains the two examples mentioned above.

Acknowledgements. This work was largely inspired by a question I was asked by E. Vasserot. I would like to thank D. Sage, M. Schlichting, and E. Vasserot for helpful and enlightening conversations. I am grateful to the referee for help with the arguments in Section 2.2

Added in revision. Since this paper first appeared in preprint form in September 2007, considerable progress has been made in the theory of staggered sheaves. Two rich classes of examples are now available: flag varieties [AS2 and toric varieties [T]. Some key results on simple objects are now known to hold in greater generality than proved here [AT1]. With respect to a suitable filtration of the derived category [AT1], staggered sheaves obey "purity" and "decomposition" theorems [AT2, analogous to results of [BBD] for $\ell$-adic mixed perverse sheaves. Finally, every simple staggered sheaf admits both a projective cover and a "standard" cover [A2, the latter being analogous to a Verma module in category $\mathcal{O}$.

\section{Preliminaries}

2.1. Notation and assumptions. Let $X$ be a scheme of finite type over a noetherian base scheme admitting a dualizing complex in the sense of $[\mathrm{H}$, Chap. V], and let $G$ be an affine group scheme over the same base, acting on $X$. Let $\mathcal{C}(X)$ (resp. $\mathcal{Q}(X)$ ) denote the category of coherent (resp. quasicoherent) sheaves on $X$, and let $\mathcal{C}_{G}(X)$ (resp. $\mathcal{Q}_{G}(X)$ ) denote the category of $G$-equivariant coherent (resp. quasicoherent) sheaves on $X$. We also assume, following [B1, that $G$ is flat, of finite type, and Gorenstein over the base scheme, and that both $\mathcal{C}(X)$ and $\mathcal{C}_{G}(X)$ have enough locally free objects.

The terms "subscheme," "sheaf," and "vector bundle" should always be understood to mean "G-invariant subscheme," "G-equivariant coherent sheaf," and "G-equivariant vector bundle," unless explicitly specified otherwise. Similarly, the term "irreducible" should always be understood in the $G$-invariant sense: a scheme is irreducible if it is not a union of two proper closed $G$-invariant subschemes.

For brevity, we introduce the notation

$$
\mathcal{D}_{G}^{-}(X)=D^{-}\left(\mathcal{C}_{G}(X)\right) \quad \text { and } \quad \mathcal{D}_{G}^{\mathrm{b}}(X)=D^{\mathrm{b}}\left(\mathcal{C}_{G}(X)\right)
$$


for the bounded-above and bounded derived categories of $\mathcal{C}_{G}(X)$. These are equivalent to the full triangulated subcategories of $D^{-}\left(\mathcal{Q}_{G}(X)\right)$ and $D^{\mathrm{b}}\left(\mathcal{Q}_{G}(X)\right)$, respectively, consisting of objects with coherent cohomology. (See [B1, Corollary 1].) We also let

$$
\mathcal{D}_{G}^{+}(X)=\left\{\mathcal{F} \in D^{+}\left(\mathcal{Q}_{G}(X)\right) \mid \mathcal{F} \text { has coherent cohomology }\right\} .
$$

$\mathcal{D}_{G}^{+}(X)$ is not, in general, equivalent to $D^{+}\left(\mathcal{C}_{G}(X)\right)$, but it is the right category to work in from the viewpoint of Grothendieck-Serre duality. (We omit the subscript $G$ for the corresponding nonequivariant derived categories.) The cohomology sheaves of an object $\mathcal{F}$ in one of these derived categories will be denoted $H^{k}(\mathcal{F})$. In particular, this notation will not be used for derived functors of the global-section functor $\Gamma$.

We write $\mathcal{D}_{G}^{-}(X)^{\leq n}$ and $\mathcal{D}_{G}^{\mathrm{b}}(X)^{\leq n}$ for the full subcategories consisting objects $\mathcal{F}$ with $H^{k}(\mathcal{F})=0$ for $k>n$. $\mathcal{D}_{G}^{+}(X)^{\geq n}$ and $\mathcal{D}_{G}^{\mathrm{b}}(X)^{\geq n}$ are defined similarly, and we put $\mathcal{D}_{G}^{\mathrm{b}}(X)^{[a, b]}=\mathcal{D}_{G}^{\mathrm{b}}(X)^{\geq a} \cap \mathcal{D}_{G}^{\mathrm{b}}(X)^{\leq b}$. We also have truncation functors $\tau^{\leq n}$ and $\tau^{\geq n}$ for each $n$, and the composition $\tau^{[a, b]}=\tau^{\geq a} \circ \tau^{\leq b}$.

Let $Z \subset X$ be a closed subscheme. The notation

$$
V \Subset_{Z} X
$$

will be used to mean that $V$ is an open subscheme of $X$ containing the complement of $Z$, and with the property that $V \cap Z$ is a dense open subscheme of $Z$. (In particular, $V$ is a dense open subscheme of $X$.) We also use the notation

$$
V \Subset X
$$

as a synonym for $V \Subset_{X} X$, i.e., to say that $V$ is a dense open subscheme of $X$.

Given $\mathcal{F}, \mathcal{G} \in \mathcal{C}_{G}(X)$, we write $\mathcal{H} o m(\mathcal{F}, \mathcal{G})$ for their "internal Hom," also an object of $\mathcal{C}_{G}(X)$. This $\mathcal{H}$ om commutes with the forgetful functor to the nonequivariant category. Note that $\Gamma(\mathcal{H o m}(\mathcal{F}, \mathcal{G})) \neq \operatorname{Hom}(\mathcal{F}, \mathcal{G})$ in general, as the latter is the group of $G$-equivariant morphisms.

Next, we define $\operatorname{Hom}(\mathcal{F}, \mathcal{G})$ to be the rule assigning to each open subscheme $V \subset X$ the group $\operatorname{Hom}\left(\left.\mathcal{F}\right|_{V},\left.\mathcal{G}\right|_{V}\right)$. This is not usually a sheaf, as it does not take values on non- $G$-invariant open subschemes. We define $\operatorname{Ext}^{r}(\mathcal{F}, \mathcal{G})$ for any $r \geq 0$ similarly. We will not formally develop the theory of such objects; rather, the main use of this notation will be to enable us abbreviate certain kinds of statements and arguments. For instance, we may say " $\operatorname{Hom}\left(\left.\mathcal{F}\right|_{U},\left.\mathcal{G}\right|_{U}\right)=0$ " rather than "for all $V \subset U, \operatorname{Hom}\left(\left.\mathcal{F}\right|_{V},\left.\mathcal{G}\right|_{V}\right)=0$," and we may state that there is an exact sequence

$$
\operatorname{Hom}\left(\mathcal{F}, \mathcal{G}^{\prime \prime}\right) \rightarrow \operatorname{Ext}^{1}\left(\mathcal{F}, \mathcal{G}^{\prime}\right) \rightarrow \operatorname{Ext}^{1}(\mathcal{F}, \mathcal{G})
$$

to mean that for every open subscheme $V \subset X$, there is an exact sequence

$$
\operatorname{Hom}\left(\left.\mathcal{F}\right|_{V},\left.\mathcal{G}^{\prime \prime}\right|_{V}\right) \rightarrow \operatorname{Ext}^{1}\left(\left.\mathcal{F}\right|_{V},\left.\mathcal{G}^{\prime}\right|_{V}\right) \rightarrow \operatorname{Ext}^{1}\left(\left.\mathcal{F}\right|_{V},\left.\mathcal{G}\right|_{V}\right) .
$$

If $i: Z \hookrightarrow X$ is a closed subscheme of $X$, the underlying topological space of $Z$ will be denoted $\underline{Z}$. An object $\mathcal{F}$ of $\mathcal{C}_{G}(X)$ or $\mathcal{D}_{G}(X)$ is said to be supported on $Z$ if $\mathcal{F} \simeq i_{*} \mathcal{F}_{1}$ for some object $\mathcal{F}_{1}$ of $\mathcal{C}_{G}(Z)$ or $\mathcal{D}_{G}(Z)$. On the other hand, $\mathcal{F}$ is supported on $\underline{Z}$ simply if all nonzero stalks of $\mathcal{F}$ are over points of $\underline{Z}$. We define two categories related to $\underline{Z}$ as follows:

$$
\begin{aligned}
& \mathcal{C}_{G}^{\text {supp }}(X, Z)=\left\{\mathcal{F} \in \mathcal{C}_{G}(X) \mid \mathcal{F} \text { is supported on } \underline{Z}\right\}, \\
& \mathcal{D}_{G}^{\text {supp }}(X, Z)=\left\{\mathcal{F} \in \mathcal{D}_{G}^{\mathrm{b}}(X) \mid \mathcal{F} \text { is supported on } \underline{Z}\right\} .
\end{aligned}
$$


Note that objects of $\mathcal{D}_{G}^{\text {supp }}(X, Z)$ are by definition bounded. Recall that for every object $\mathcal{F}$ in $\mathcal{C}_{G}^{\text {supp }}(X, Z)$ or $\mathcal{D}_{G}^{\text {supp }}(X, Z)$, there exists a closed subscheme structure $i_{Z^{\prime}}: Z^{\prime} \hookrightarrow X$ and an object $\mathcal{F}_{1}$ in $\mathcal{C}_{G}\left(Z^{\prime}\right)$ or $\mathcal{D}_{G}^{\mathrm{b}}\left(Z^{\prime}\right)$ such that $\mathcal{F} \simeq i_{Z^{\prime} *} \mathcal{F}_{1}$.

For a closed subscheme $i: Z \hookrightarrow X$, the notation $i^{*}$ will always denote the coherent pull-back functor $i^{*}: \mathcal{C}_{G}(X) \rightarrow \mathcal{C}_{G}(Z)$. We denote by $i^{!}: \mathcal{C}_{G}(X) \rightarrow \mathcal{C}_{G}(Z)$ the functor of "sections supported on $Z$." This functor is right adjoint to $i_{*}$ : $\mathcal{C}_{G}(Z) \rightarrow \mathcal{C}_{G}(X)$, and satisfies $i_{*} i^{!} \mathcal{F} \simeq \mathcal{H}$ om $\left(i_{*} \mathcal{O}_{Z}, \mathcal{F}\right)$. Note that $i_{*} i^{!} \mathcal{F}$ is naturally a subsheaf of $\mathcal{F}$.

Finally, we let $\Gamma_{Z}: \mathcal{C}_{G}(X) \rightarrow \mathcal{C}_{G}(X)$ be the functor of "sections supported on $\underline{Z}$." $\Gamma_{Z} \mathcal{F}$ is a subsheaf of $\mathcal{F}$, and we have a natural isomorphism

$$
\Gamma_{Z} \mathcal{F} \simeq \lim _{\overrightarrow{Z^{\prime}}} i_{Z^{\prime}} i_{Z^{\prime}} \mathcal{F}
$$

where $i_{Z^{\prime}}: Z^{\prime} \hookrightarrow X$ ranges over all closed subscheme structures on $\underline{Z}$.

2.2. Equivariant derived categories. There are a number of foundational issues to be addressed in translating the theory of derived categories and functors of coherent sheaves from the nonequivariant setting (following $[\mathrm{H}]$ ) to the equivariant one. Many of these have been treated by Bezrukavnikov [B1, Section 2]. Here, we briefly consider the issues that are most relevant to the present paper. Consider first the derived functors

$$
L i^{*}: \mathcal{D}_{G}^{-}(X) \rightarrow \mathcal{D}_{G}^{-}(Z) \quad \text { and } \quad R \mathcal{H} \text { om }:\left(\mathcal{D}_{G}^{-}(X)\right)^{\mathrm{op}} \times \mathcal{D}_{G}^{+}(X) \rightarrow \mathcal{D}_{G}^{+}(X) .
$$

$L i^{*}$ may be computed by taking a locally free resolution, since $\mathcal{C}_{G}(X)$ is assumed to have enough locally free objects. Similarly, $R \mathcal{H}$ om may be computed by taking a locally free resolution in the first variable. We will also require the derived functor of $i$. The quasicoherent category $\mathcal{Q}_{G}(X)$ has enough injectives, so there is no problem in constructing the functor

$$
R i^{!}: D^{+}\left(\mathcal{Q}_{G}(X)\right) \rightarrow D^{+}\left(\mathcal{Q}_{G}(Z)\right),
$$

but it is not yet clear how to obtain from this a functor on $\mathcal{D}_{G}^{+}(X)$. We will address this issue below.

Lemma 2.1. Let $j: U \hookrightarrow X$ be an open subscheme, and let $i: Z \hookrightarrow X$ be a complementary closed subscheme.

(1) The functor $L i^{*}$ is left-adjoint to $i_{*}$, and $R i^{!}$is right-adjoint to $i_{*}$.

(2) The functors $L i^{*}, R \mathcal{H}$ om, and $R i^{!}$commute with the forgetful functor to the appropriate nonequivariant derived category. In particular, $R i^{!}$restricts to a functor $\mathcal{D}_{G}^{+}(X) \rightarrow \mathcal{D}_{G}^{+}(Z)$.

(3) For any $\mathcal{F} \in \mathcal{D}_{G}^{-}(X)$ and $\mathcal{G} \in \mathcal{D}_{G}^{+}(X)$, there is a long exact sequence

$$
\rightarrow \lim _{\overrightarrow{Z^{\prime}}} \operatorname{Hom}\left(L i_{Z^{\prime}}^{*} \mathcal{F}, R i_{Z^{\prime}}^{\prime} \mathcal{G}\right) \rightarrow \operatorname{Hom}(\mathcal{F}, \mathcal{G}) \rightarrow \operatorname{Hom}\left(j^{*} \mathcal{F}, j^{*} \mathcal{G}\right) \rightarrow
$$

where the limit runs over all subscheme structures $i_{Z^{\prime}}: Z^{\prime} \hookrightarrow X$ on $\underline{Z}$.

Proof. (11) The adjointness properties for $L i^{*}, R i^{!}$, and $i_{*}$ follow by standard arguments from the corresponding adjointness properties at the level of abelian categories. (For now, the adjointness of $i_{*}$ and $R i^{!}$holds only in the quasicoherent setting.)

(2) Because both $L i^{*}$ and $R \mathcal{H}$ om can be computed by locally free resolutions in both the equivariant and the nonequivariant cases, the fact that they commute 
with the forgetful functor $F$ follows from the fact that $F$ takes locally free sheaves in $\mathcal{C}_{G}(X)$ to locally free sheaves in $\mathcal{C}(X)$.

Before dealing with $R i^{1}$, we first consider another approach to computing $R \mathcal{H}$ om, via certain resolutions in the second variable. The subtlety here is that $\mathrm{F}$ does not, in general, take injective objects of $\mathcal{Q}_{G}(X)$ to injective objects of $\mathcal{Q}(X)$. To get around this problem, we make use of the "averaging functor" $\mathrm{Av}=a_{*} p r^{*}$ : $\mathcal{Q}(X) \rightarrow \mathcal{Q}_{G}(X)$, where $p r: G \times X \rightarrow X$ is projection on to the second factor, and $a: G \times X \rightarrow X$ is the action map. Av is exact and right-adjoint to $\mathrm{F}$, and it takes injective objects to injective objects (see [B1, Section 2]). Let us say that an object of $\mathcal{Q}_{G}(X)$ is $\mathrm{Av}$-injective if it is isomorphic to $\operatorname{Av}(\mathcal{I})$ for some injective object $\mathcal{I} \in \mathcal{Q}(X)$. Then every object of $\mathcal{Q}_{G}(X)$ is a subsheaf of some Av-injective sheaf, and every object in $D^{+}\left(\mathcal{Q}_{G}(X)\right)$ admits an $A v$-injective resolution. We claim that $R \mathcal{H}$ om can be computed by $A v$-injective resolutions in the second variable. This claim would follow from the statement that if $\mathcal{I} \in \mathcal{Q}(X)$ is injective, then $\mathcal{H o m}(\cdot, \operatorname{Av}(\mathcal{I}))$ is an exact functor on $\mathcal{Q}_{G}(X)$. The latter holds because

$$
\mathcal{H o m}(\cdot, \operatorname{Av}(I)) \simeq \operatorname{Av}(\mathcal{H o m}(\mathrm{F}(\cdot), \mathcal{I})),
$$

and because $\mathcal{H} o m(\cdot, \mathcal{I})$ is an exact functor on $\mathcal{Q}(X)$.

Now, for a coherent sheaf $\mathcal{F} \in \mathcal{C}_{G}(X)$, consider the sheaf of abelian groups on $Z$ given by

$$
\left.\mathcal{H o m}\left(i_{*} \mathcal{O}_{Z}, \mathcal{F}\right)\right|_{Z}
$$

Here, "I $Z$ " denotes the exact functor of restriction to $Z$ in the usual sense for sheaves of abelian groups, not the coherent pull-back functor. Nevertheless, it easy to see that this sheaf can be naturally regarded as an object of $\mathcal{C}_{G}(Z)$. Indeed, because $i_{*} i^{i} \mathcal{F} \simeq \mathcal{H} o m\left(i_{*} \mathcal{O}_{Z}, c F\right)$, we have

$$
\left.i^{!} \mathcal{F} \simeq \mathcal{H o m}\left(i_{*} \mathcal{O}_{Z}, \mathcal{F}\right)\right|_{Z} .
$$

We can use this formula to better understand $R i^{!}$. For $\mathcal{F} \in \mathcal{D}_{G}^{+}(X), R i^{!} \mathcal{F}$ can be computed by applying $\left.i^{!} \simeq \mathcal{H} \operatorname{om}\left(i_{*} \mathcal{O}_{Z}, \cdot\right)\right|_{Z}$ to an Av-injective resolution of $\mathcal{F}$. By the previous paragraph, this is equivalent to taking a chain complex representing $R \mathcal{H o m}\left(i_{*} \mathcal{O}_{Z}, \mathcal{F}\right)$ and then applying " ${ }_{Z}$." Since $R \mathcal{H}$ om and restriction to $Z$ both commute with $\mathrm{F}, R i^{!}$does as well. In particular, when evaluated on objects of $\mathcal{D}_{G}^{+}(X), R i^{!}$takes values in $\mathcal{D}_{G}^{+}(Z)$ (i.e., it has coherent cohomology), because the analogous statement is true for the corresponding nonequivariant functor.

(3) We follow the proof given in [B1, Proposition 2]. In $D^{+}\left(\mathcal{Q}_{G}(X)\right)$, there is a well-known distinguished triangle $R \Gamma_{Z} \mathcal{G} \rightarrow \mathcal{G} \rightarrow R j_{*} j^{*} \mathcal{G} \rightarrow$, from which we obtain a long exact sequence

$$
\rightarrow \operatorname{Hom}\left(\mathcal{F}, R \Gamma_{Z} \mathcal{G}\right) \rightarrow \operatorname{Hom}(\mathcal{F}, \mathcal{G}) \rightarrow \operatorname{Hom}\left(\mathcal{F}, R j_{*} j^{*} \mathcal{G}\right) \rightarrow
$$

By [B1, Lemma 3(b)], we have

$$
\operatorname{Hom}\left(\mathcal{F}, R \Gamma_{Z} \mathcal{G}\right) \simeq \lim _{\overrightarrow{Z^{\prime}}} \operatorname{Hom}\left(\mathcal{F}, i_{Z^{\prime} *} R i_{Z^{\prime}}^{!} \mathcal{G}\right) \simeq \lim _{\overrightarrow{Z^{\prime}}} \operatorname{Hom}\left(L i_{Z^{\prime}}^{*} \mathcal{F}, R i_{Z^{\prime}}^{\prime} \mathcal{G}\right),
$$

where $i_{Z^{\prime}}: Z^{\prime} \hookrightarrow X$ runs over all subscheme structures on $\underline{Z}$, and on the other hand, we have $\operatorname{Hom}\left(\mathcal{F}, R j_{*} j^{*} \mathcal{G}\right) \simeq \operatorname{Hom}\left(j^{*} \mathcal{F}, j^{*} \mathcal{G}\right)$.

According to [B1, Proposition 1], the scheme $X$ admits an equivariant dualizing complex, i.e., an object $\omega_{X} \in \mathcal{D}_{G}^{\mathrm{b}}(X)$ such that the functor $\mathbb{D}=R \mathcal{H}$ om $\left(\cdot, \omega_{X}\right)$ : $\mathcal{D}_{G}^{\mathrm{b}}(X) \rightarrow \mathcal{D}_{G}^{\mathrm{b}}(X)$ has the property that $\mathbb{D} \circ \mathbb{D} \simeq \mathrm{id}$. The functor $\mathbb{D}$ can be evaluated on objects of $\mathcal{D}_{G}^{-}(X)$; it then takes values in $\mathcal{D}_{G}^{+}(X)$. 
One can also evaluate $\mathbb{D}$ on objects of $\mathcal{D}_{G}^{+}(X)$ : remarkably, its values, a priori belonging to the unbounded derived category $D\left(\mathcal{Q}_{G}(X)\right)$, actually lie in $\mathcal{D}_{G}^{-}(X)$. This fact follows from the corresponding nonequivariant statement, since we know that $R \mathcal{H}$ om commutes with $\mathrm{F}$, and $\mathrm{F}\left(\omega_{X}\right)$ is a nonequivariant dualizing complex [B1, Lemma 4]. The nonequivariant version holds because $F\left(\omega_{X}\right)$ is quasi-isomorphic to a bounded complex of injective objects of $\mathcal{Q}(X)[\mathrm{H}$, II.7.20]. (Note that the latter argument cannot simply be repeated in the equivariant case: $\omega_{X}$ need not be quasiisomorphic to a bounded complex of injective objects of $\mathcal{Q}_{G}(X)$.)

The functor $\mathbb{D}$ gives antiequivalences in both directions:

$$
\mathbb{D}: \mathcal{D}_{G}^{-}(X) \rightarrow \mathcal{D}_{G}^{+}(X), \quad \mathbb{D}: \mathcal{D}_{G}^{+}(X) \rightarrow \mathcal{D}_{G}^{-}(X) .
$$

In general, dualizing complexes are not uniquely determined, but if we are given a dualizing complex $\omega_{X}$ on $X$, we can construct from it a dualizing complex on any open subscheme $j: U \hookrightarrow X$ by the formula

$$
\omega_{U}=\left.\omega_{X}\right|_{U}
$$

On the other hand, for a closed subscheme $i: Z \hookrightarrow X$, the object

$$
\omega_{Z}=R i \omega_{X}
$$

is an equivariant dualizing complex. (Again, by Lemma 2.1 and [B1, Lemma 4], this statement follows from the corresponding nonequivariant one, proved in $[\mathrm{H}$, V.2.4].) In particular, $\omega_{Z}$ is necessarily in $\mathcal{D}_{G}^{\mathrm{b}}(Z)$. From Section 6 on, we will have a fixed dualizing complex $\omega_{X}$ in mind for $X$, and we adopt the convention that on any locally closed subscheme of $X, \mathbb{D}$ is to be computed with the dualizing complex obtained from $\omega_{X}$ by the above formulas.

Lemma 2.2. The functor $\mathbb{D}$ commutes with the forgetful functor to the nonequivariant derived category. For a closed subscheme $i: Z \hookrightarrow X$, we have $\mathbb{D} \circ L i^{*} \simeq R i^{!} \circ \mathbb{D}$.

Proof. The first part of the lemma is immediate from Lemma 2.1. Next, recall that for $\mathcal{F}$ and $\mathcal{G}$ in $\mathcal{C}_{G}(X)$, we have a natural isomorphism

$$
\mathcal{H o m}\left(i^{*} \mathcal{F}, i^{!} \mathcal{G}\right) \simeq i^{!} \mathcal{H o m}(\mathcal{F}, \mathcal{G}) .
$$

By the usual general methods (see [H, Proposition III.6.9(b)] for the nonequivariant case), we may deduce that

$$
R \mathcal{H o m}\left(L i^{*} \mathcal{F}, R i^{!} \mathcal{G}\right) \simeq R i^{!} R \mathcal{H} \operatorname{om}(\mathcal{F}, \mathcal{G})
$$

for $\mathcal{F} \in \mathcal{D}_{G}^{-}(X)$ and $\mathcal{G} \in \mathcal{D}_{G}^{+}(X)$. Now, take $\mathcal{G}=\omega_{X}$. In this case, we know that $R i^{!} \omega_{X} \simeq \omega_{Z}$. The isomorphism above then becomes $\mathbb{D} \circ L i^{*} \simeq R i^{!} \circ \mathbb{D}$.

2.3. Coherent sheaves on open subschemes. We now prove several useful lemmas relating coherent sheaves on $X$ to those on an open subscheme.

Lemma 2.3. Let $j: U \hookrightarrow X$ be an open subscheme, and let $\mathcal{F} \in \mathcal{D}_{G}^{\mathrm{b}}(X)^{[a, b]}$. Given a distinguished triangle

$$
\left.\mathcal{F}_{U}^{\prime} \rightarrow \mathcal{F}\right|_{U} \rightarrow \mathcal{F}_{U}^{\prime \prime} \rightarrow
$$

in $\mathcal{D}_{G}^{\mathrm{b}}(U)$, with $\mathcal{F}_{U}^{\prime} \in \mathcal{D}_{G}^{\mathrm{b}}(U)^{[a, b]}$, there exist objects $\mathcal{F}^{\prime} \in \mathcal{D}_{G}^{\mathrm{b}}(X)^{[a, b]}, \mathcal{F}^{\prime \prime} \in \mathcal{D}_{G}^{\mathrm{b}}(X)$ and a distinguished triangle

$$
\mathcal{F}^{\prime} \rightarrow \mathcal{F} \rightarrow \mathcal{F}^{\prime \prime} \rightarrow
$$

in $\mathcal{D}_{G}^{\mathrm{b}}(X)$ whose image under $j^{*}$ is isomorphic to the distinguished triangle given in (2.1). 
Proof. Fix a bounded complex $\mathcal{G}^{\bullet}$ of objects of $\mathcal{C}_{G}(X)$ that represents $\mathcal{F}$ and such that $\mathcal{G}^{i}=0$ for $i<a$ or $i>b$. Then, there exists a complex $\mathcal{G}_{U}^{\prime} \bullet^{\bullet}$ of objects of $\mathcal{C}_{G}(U)$ and a morphism of complexes $f:\left.\mathcal{G}_{U}^{\prime} \rightarrow \mathcal{G}^{\bullet}\right|_{U}$ representing the morphism $\left.\mathcal{F}_{U}^{\prime} \rightarrow \mathcal{F}\right|_{U}$. We may again assume that $\mathcal{G}_{U}^{\prime}{ }^{i}=0$ for $i<a$ or $i>b$. Now, $f$ induces a morphism of complexes of quasicoherent sheaves $j_{*} f: j_{*} \mathcal{G}_{U}^{\prime} \bullet \rightarrow j_{*} j^{*} \mathcal{G}^{\bullet}$. There is also a natural morphism $\mathcal{G}^{\bullet} \rightarrow j_{*} j^{*} \mathcal{G}^{\bullet}$. Consider the diagram

$$
j_{*} \mathcal{G}_{U}^{\prime} \stackrel{\mathcal{G}^{\bullet}}{\downarrow} \stackrel{j_{*} f}{\rightarrow} j_{*} j^{*} \mathcal{G}^{\bullet}
$$

There certainly exist coherent subcomplexes of $j_{*} \mathcal{G}_{U}^{\prime} \bullet$ whose restriction to $U$ is $\mathcal{G}_{U}^{\prime} \bullet$. Choose one such complex, and denote it $\mathcal{H}_{1}^{\bullet}$. Now, form the pullback

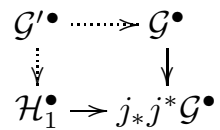

When we apply the exact functor $j^{*}$, the right-hand vertical arrow becomes an isomorphism, and after passing to $\mathcal{D}_{G}^{\mathrm{b}}(U)$, the arrow along the bottom becomes $\left.\mathcal{F}_{U}^{\prime} \rightarrow \mathcal{F}\right|_{U}$. Therefore, the left-hand vertical arrow also becomes an isomorphism, and $j^{*} \mathcal{G}^{\prime} \bullet \rightarrow j^{*} \mathcal{G}^{\bullet}$ is isomorphic in $\mathcal{D}_{G}^{\mathrm{b}}(U)$ to $\left.\mathcal{F}_{U}^{\prime} \rightarrow \mathcal{F}\right|_{U}$. Let $\mathcal{F}^{\prime}$ be $\mathcal{G}^{\bullet}$ regarded as an object of $\mathcal{D}_{G}^{\mathrm{b}}(X)$, and let $\mathcal{F}^{\prime \prime}$ be the cone of $\mathcal{F}^{\prime} \rightarrow \mathcal{F}$. Then $\mathcal{F}^{\prime} \rightarrow \mathcal{F} \rightarrow \mathcal{F}^{\prime \prime} \rightarrow$ is the distinguished triangle we seek.

When $a=b=0$, the previous lemma yields the following abelian-category statement.

Lemma 2.4. Let $U \subset X$ be an open subscheme. Let $\mathcal{F} \in \mathcal{C}_{G}(X)$. For any short exact sequence $\left.0 \rightarrow \mathcal{F}_{U}^{\prime} \rightarrow \mathcal{F}\right|_{U} \rightarrow \mathcal{F}_{U}^{\prime} \rightarrow 0$ of sheaves on $U$, there exists a short exact sequence $0 \rightarrow \mathcal{F}^{\prime} \rightarrow \mathcal{F} \rightarrow \mathcal{F}^{\prime \prime} \rightarrow 0$ of sheaves on $X$ whose image under $j^{*}$ is the given short exact sequence.

Lemma 2.5. Let $\mathcal{C}$ be a full subcategory of $\mathcal{C}_{G}(X)$, closed under subobjects. Let $i: Z \hookrightarrow X$ be a closed subscheme, and let $j: U \hookrightarrow X$ be the complementary open subscheme. If $\mathcal{G} \in \mathcal{C}_{G}(X)$ is such that $\operatorname{Hom}\left(\mathcal{F}, \mathcal{G} / \Gamma_{Z} \mathcal{G}\right)=0$ for all $\mathcal{F} \in \mathcal{C}$, then $\operatorname{Hom}\left(j^{*} \mathcal{F}, j^{*} \mathcal{G}\right)=0$ for all $\mathcal{F} \in \mathcal{C}$.

Proof. Suppose $\operatorname{Hom}\left(j^{*} \mathcal{F}, j^{*} \mathcal{G}\right) \neq 0$ for some $\mathcal{F} \in \mathcal{C}$. Then there is a nonzero morphism of quasicoherent sheaves $f: j_{*} j^{*} \mathcal{F} \rightarrow j_{*} j^{*} \mathcal{G}$ such that $j^{*} f$ is also nonzero. Now, consider the following diagram

$$
\begin{array}{r}
\mathcal{F} \stackrel{p}{\rightarrow} j_{*} j^{*} \mathcal{F} \\
\Gamma_{Z} \mathcal{G} \rightarrow \mathcal{G} \rightarrow \stackrel{q}{\downarrow_{f}} j_{*} j^{*} \mathcal{G}
\end{array}
$$

The bottom row is left exact, and in particular, the image of $q$ is isomorphic to $\mathcal{G} / \Gamma_{Z} \mathcal{G}$. Now, let $\mathcal{H}=\operatorname{im}(f \circ p)$. This is a coherent subsheaf of $j_{*} j^{*} \mathcal{G}$. Moreover, $\mathcal{H} \cap \operatorname{im} q$ must be nonzero, because when we apply the exact functor $j^{*}$ to this diagram, we find that $j^{*} \mathcal{H} \simeq \operatorname{im}\left(j^{*} f\right)$ is a nonzero subsheaf of $j^{*}\left(j_{*} j^{*} \mathcal{G}\right) \simeq j^{*} \mathcal{G}$. Let $\mathcal{F}^{\prime} \subset \mathcal{F}$ be the preimage via $f \circ p$ of $\mathcal{H} \cap \mathrm{im} q$. This is a nonzero subsheaf of $\mathcal{F}$, and so a member of $\mathcal{C}$. The restriction of $f \circ p$ to $\mathcal{F}^{\prime}$ is a nonzero morphism $\mathcal{F}^{\prime} \rightarrow \operatorname{im} q \simeq \mathcal{G} / \Gamma_{Z} \mathcal{G}$, a contradiction. 
2.4. Abelian categories. We conclude this section with two useful propositions about abelian categories. Recall that an abelian category $\mathcal{A}$ is said to be noetherian if for every object $A \in \mathcal{A}$, the ascending chain condition holds for subobjects of $A$.

Proposition 2.6. Let $\mathcal{A}$ be a noetherian abelian category, and let $\mathcal{A}^{\prime}$ be a full subcategory closed under extensions and quotients. Then the inclusion functor $\iota$ : $\mathcal{A}^{\prime} \rightarrow \mathcal{A}$ admits a right adjoint $\rho: \mathcal{A} \rightarrow \mathcal{A}^{\prime}$.

Proof. Given $A \in \mathcal{A}$, consider the set $S$ of all subobjects of $A$ belonging to $\mathcal{A}^{\prime} . S$ is partially ordered by inclusion, and totally ordered subsets of $S$ have a maximum element, by the ascending chain condition. By Zorn's lemma, $S$ contains at least one maximal element.

Now, suppose $S$ contained two distinct maximal elements, $B_{1}$ and $B_{2}$. Form the cartesian and cocartesian square

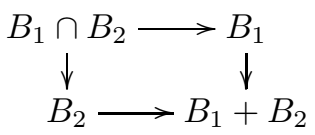

Now, $B_{1} /\left(B_{1} \cap B_{2}\right) \simeq\left(B_{1}+B_{2}\right) / B_{1}$ is in $\mathcal{A}^{\prime}$, since $\mathcal{A}^{\prime}$ is closed under quotients. But $B_{1}+B_{2}$ is an extension of $\left(B_{1}+B_{2}\right) / B_{1}$ by $B_{1}$, and since $\mathcal{A}^{\prime}$ is closed under extensions, $B_{1}+B_{2} \in \mathcal{A}^{\prime}$. This contradicts the maximality of $B_{1}$ and $B_{2}$ in $S$, so $S$ cannot contain two distinct maximal elements.

Therefore, $S$ contains a unique maximal element $A^{\prime}$. Now, let $B \in \mathcal{A}^{\prime}$. For any morphism $f: B \rightarrow A$, the image of $f$ is a subobject of $A$ that is in $\mathcal{A}^{\prime}$, since $\mathcal{A}^{\prime}$ is closed under quotients. Therefore, the image of $f$ is actually a subobject of $A^{\prime}$, and $f$ factors through the inclusion $A^{\prime} \hookrightarrow A$. We have shown that

$$
\operatorname{Hom}(B, A) \simeq \operatorname{Hom}\left(B, A^{\prime}\right),
$$

so the functor $\rho: A \mapsto A^{\prime}$ is the desired right adjoint.

Next, recall that the (right) orthogonal complement of a subcategory $\mathcal{A}^{\prime} \subset \mathcal{A}$ is the full subcategory defined by

$$
\left(\mathcal{A}^{\prime}\right)^{\perp}=\left\{B \mid \operatorname{Hom}(A, B)=0 \text { for all } A \in \mathcal{A}^{\prime}\right\} .
$$

Proposition 2.7. Let $\mathcal{A}$ be a noetherian abelian category, and let $\mathcal{A}^{\prime}$ be a full subcategory closed under extensions and quotients. If $\mathcal{A}^{\prime \prime}$ is full subcategory of $\mathcal{A}$ contained in $\left(\mathcal{A}^{\prime}\right)^{\perp}$, then the following conditions are equivalent:

(1) $\mathcal{A}^{\prime \prime}=\left(\mathcal{A}^{\prime}\right)^{\perp}$.

(2) For every object $A \in \mathcal{A}$, there is a short exact sequence

$$
0 \rightarrow A^{\prime} \rightarrow A \rightarrow A^{\prime \prime} \rightarrow 0
$$

with $A^{\prime} \in \mathcal{A}^{\prime}$ and $A^{\prime \prime} \in \mathcal{A}^{\prime \prime}$.

When these conditions hold, the inclusion functor $\mathcal{A}^{\prime \prime} \rightarrow \mathcal{A}$ admits a left adjoint $\lambda: \mathcal{A} \rightarrow \mathcal{A}^{\prime \prime}$. Moreover, for every $A \in \mathcal{A}$, there is a natural short exact sequence

$$
0 \rightarrow \rho(A) \rightarrow A \rightarrow \lambda(A) \rightarrow 0,
$$

where $\rho$ is the right adjoint to the inclusion $\mathcal{A}^{\prime} \rightarrow \mathcal{A}$, and in addition every short exact sequence of the form (2.2) is canonically isomorphic to this one. 
Proof. For any $A \in \mathcal{A}$, we claim that $A / \rho(A) \in\left(\mathcal{A}^{\prime}\right)^{\perp}$. Indeed, if there were a nonzero morphism $A_{1} \rightarrow A / \rho(A)$ with $A_{1} \in \mathcal{A}^{\prime}$, then its image im $f$ would be a subobject of $A / \rho(A)$ in $\mathcal{A}^{\prime}$. Since $\mathcal{A}^{\prime}$ is stable under extensions, the preimage in $A$ of im $f$ (an extension of im $f$ by $\rho(A)$ ) would be a subobject of $A$ in $\mathcal{A}^{\prime}$ containing $\rho(A)$ as a proper subobject. But that contradicts the characterization of $\rho(A)$ as the maximal subobject of $A$ in $\mathcal{A}^{\prime}$.

It is now clear that condition (11) implies condition (2): for any $A \in \mathcal{A}$, the short exact sequence

$$
0 \rightarrow \rho(A) \rightarrow A \rightarrow A / \rho(A) \rightarrow 0
$$

is of the form (2.2).

Conversely, suppose condition (2) holds. Given $A \in\left(\mathcal{A}^{\prime}\right)^{\perp}$, we must show that $A \in \mathcal{A}^{\prime \prime}$. Form a short exact sequence

$$
0 \rightarrow A^{\prime} \rightarrow A \rightarrow A^{\prime \prime} \rightarrow 0
$$

as in (2.2). Now, the morphism $A^{\prime} \rightarrow A$ must be 0 , since $A \in\left(\mathcal{A}^{\prime}\right)^{\perp}$, so in fact $A \simeq A^{\prime \prime} \in \mathcal{A}^{\prime \prime}$, as desired.

We now prove the last part of the proposition. Given $A \in \mathcal{A}$, form a short exact sequence as in (2.2). Now, for any $B \in \mathcal{A}^{\prime \prime}$, we have an exact sequence

$$
0 \rightarrow \operatorname{Hom}\left(A^{\prime \prime}, B\right) \rightarrow \operatorname{Hom}(A, B) \rightarrow \operatorname{Hom}\left(A^{\prime}, B\right) .
$$

The last term vanishes because $\mathcal{A}^{\prime \prime} \subset\left(\mathcal{A}^{\prime}\right)^{\perp}$, so if we put $\lambda(A)=A^{\prime \prime}$, we have an isomorphism $\operatorname{Hom}(\lambda(A), B) \stackrel{\sim}{\rightarrow} \operatorname{Hom}(A, B)$. Now, we know that $A^{\prime} \rightarrow A$ must factor through $\rho(A) \rightarrow A$, so $\rho(A) / A^{\prime}$ can be identified with a subobject of $A^{\prime \prime}$. But $\rho(A) / A^{\prime} \in \mathcal{A}^{\prime}$, and since $A^{\prime \prime} \in\left(\mathcal{A}^{\prime}\right)^{\perp}$, we must have $\rho(A) \simeq A^{\prime}$, and hence that $\lambda(A) \simeq A / \rho(A)$. This proves the functoriality of $\lambda$ (which is now clearly left adjoint to $\mathcal{A}^{\prime \prime} \rightarrow \mathcal{A}$ ) and the uniqueness of the short exact sequence (2.2).

\section{3. $s$-STRUCTURES ON SCHEMES}

In this section, we define and establish basic properties of $s$-structures. The definition is quite lengthy and is given in several steps, partly because it will be convenient in the sequel to be able to discuss situations in which only part of the full definition is satisfied.

Definition 3.1. A pre-s-structure on $X$ is a collection of full subcategories

$$
\left(\left\{\mathcal{C}_{G}(X)_{\leq w}\right\},\left\{\mathcal{C}_{G}(X)_{\geq w}\right\}\right)_{w \in \mathbb{Z}}
$$

of $\mathcal{C}_{G}(X)$ such that:

(S1) Each $\mathcal{C}_{G}(X)_{\leq w}$ is a Serre subcategory, and each $\mathcal{C}_{G}(X)_{\geq w}$ is closed under subobjects and extensions.

(S2) $\mathcal{C}_{G}(X)_{\leq w} \subset \mathcal{C}_{G}(X)_{\leq w+1}$ and $\mathcal{C}_{G}(X)_{\geq w} \supset \mathcal{C}_{G}(X)_{\geq w+1}$.

(S3) If $\mathcal{F} \in \overline{\mathcal{C}}_{G}(X)_{\leq w}$ and $\mathcal{G} \in \mathcal{C}_{G}(X)_{\geq w+1}$, then $\operatorname{Hom}(\mathcal{F}, \mathcal{G})=0$.

(S4) For any $\mathcal{F} \in \mathcal{C}_{G}(X)$ and any $w \in \mathbb{Z}$, there is a short exact sequence

$$
0 \rightarrow \mathcal{F}^{\prime} \rightarrow \mathcal{F} \rightarrow \mathcal{F}^{\prime \prime} \rightarrow 0
$$

with $\mathcal{F}^{\prime} \in \mathcal{C}_{G}(X)_{\leq w}$ and $\mathcal{F}^{\prime \prime} \in \mathcal{C}_{G}(X)_{\geq w+1}$.

(S5) For any $\mathcal{F} \in \mathcal{C}_{G}(X)$, there are integers $w, v \in \mathbb{Z}$ such that $\mathcal{F} \in \mathcal{C}_{G}(X)_{\geq w}$ and $\mathcal{F} \in \mathcal{C}_{G}(X)_{\leq v}$.

(S6) If $\mathcal{F} \in \mathcal{C}_{G}(X)_{\leq w}$ and $\mathcal{G} \in \mathcal{C}_{G}(X)_{\leq v}$, then $\mathcal{F} \otimes \mathcal{G} \in \mathcal{C}_{G}(X)_{\leq w+v}$. 
By Propositions 2.6 and 2.7, the inclusion functors $\mathcal{C}_{G}(X)_{\leq w} \rightarrow \mathcal{C}_{G}(X)$ and $\mathcal{C}_{G}(X)_{\geq w} \rightarrow \mathcal{C}_{G}(X)$ admit right and left adjoints, respectively. We denote these by $\sigma_{\leq w}: \mathcal{C}_{G}(X) \rightarrow \mathcal{C}_{G}(X)_{\leq w}$ and $\sigma_{\geq w}: \mathcal{C}_{G}(X) \rightarrow \mathcal{C}_{G}(X)_{\geq w}$. Thus, for any $w$, there is a short exact sequence

$$
0 \rightarrow \sigma_{\leq w} \mathcal{F} \rightarrow \mathcal{F} \rightarrow \sigma_{\geq w+1} \mathcal{F} \rightarrow 0 .
$$

From the proof of Proposition 2.6, we see that we may regard $\sigma_{\leq w} \mathcal{F}$ as being the largest subsheaf of $\mathcal{F}$ in $\mathcal{C}_{G}(X)_{\leq w}$.

Remark 3.2. Suppose $\left(\left\{\mathcal{C}_{G}(X)_{\leq w}\right\},\left\{\mathcal{C}_{G}(X)_{\geq w}\right\}\right)_{w \in \mathbb{Z}}$ is a collection of full subcategories of $\mathcal{C}_{G}(X)$ satisfiying axioms (S1) $-(\mathrm{S} 4)$ above. Then axiom (S6) is equivalent to the following useful condition:

(S6]) If $\mathcal{F} \in \mathcal{C}_{G}(X)_{\leq w}$ and $\mathcal{G} \in \mathcal{C}_{G}(X)_{\geq v}$, then $\mathcal{H o m}(\mathcal{F}, \mathcal{G}) \in \mathcal{C}_{G}(X)_{\geq v-w}$. Indeed, suppose axiom ( $(\underline{6} 6)$ holds, and suppose $\mathcal{F} \in \mathcal{C}_{G}(X)_{\leq w}$ and $\mathcal{G} \in \mathcal{C}_{G}(X)_{\geq v}$. By Proposition 2.7 we know that $\mathcal{C}_{G}(X)_{\geq v-w}=\mathcal{C}_{G}(X)_{\leq v-w-1}^{\perp}$, so to show that $\mathcal{H} o m(\mathcal{F}, \mathcal{G}) \in \mathcal{C}_{G}(X)_{\geq v-w}$ is equivalent to showing that

$$
\operatorname{Hom}(\mathcal{H}, \mathcal{H o m}(\mathcal{F}, \mathcal{G}))=0 \quad \text { for all } \mathcal{H} \in \mathcal{C}_{G}(X)_{\leq v-w-1} .
$$

But $\operatorname{Hom}(\mathcal{H}, \mathcal{H} o m(\mathcal{F}, \mathcal{G})) \simeq \operatorname{Hom}(\mathcal{H} \otimes \mathcal{F}, \mathcal{G})=0$ because $\mathcal{H} \otimes \mathcal{F} \in \mathcal{C}_{G}(X)_{\leq v-1}$. The same arugment shows that condition ( $\mathrm{S} 6$ ) implies axiom ( $\mathrm{S} 6$ ) as well.

Definition 3.3. Suppose $X$ has a pre-s-structure, and let $\mathcal{F} \in \mathcal{C}_{G}(X)$. If there is an integer $w$ such that $\mathcal{F} \in \mathcal{C}_{G}(Z)_{\leq w}$ and $\sigma_{\leq w-1} \mathcal{F}=0$, then $\mathcal{F}$ is said to be pure of step $w$, and we write $w=\operatorname{step} \mathcal{F}$.

Definition 3.4. Let $X$ and $Y$ be two schemes with pre-s-structures. A functor $F$ : $\mathcal{C}_{G}(X) \rightarrow \mathcal{C}_{G}(Y)$ is said to be left (resp. right) s-exact if $F\left(\mathcal{C}_{G}(X)_{\geq w}\right) \subset \mathcal{C}_{G}(Y)_{\geq w}$ (resp. $\left.F\left(\mathcal{C}_{G}(X)_{\leq w}\right) \subset \mathcal{C}_{G}(Y)_{\leq w}\right)$. It is $s$-exact if it is both left $s$-exact and right $s$-exact.

Next, we show that a pre-s-structure gives rise to canonical pre-s-structures on any open or closed (and hence any locally closed) subscheme.

Proposition 3.5. Let $j: U \hookrightarrow X$ be an open subscheme. Given a pre-s-structure on $X$, there is a unique pre-s-structure on $U$ such that $j^{*}$ is s-exact. It is given by

$$
\begin{aligned}
& \mathcal{C}_{G}(U)_{\leq w}=\left\{\mathcal{F} \mid \text { there exists an } \mathcal{F}_{1} \in \mathcal{C}_{G}(X)_{\leq w} \text { such that } j^{*} \mathcal{F}_{1} \simeq \mathcal{F}\right\}, \\
& \mathcal{C}_{G}(U)_{\geq w}=\left\{\mathcal{F} \mid \text { there exists an } \mathcal{F}_{1} \in \mathcal{C}_{G}(X)_{\leq w} \text { such that } j^{*} \mathcal{F}_{1} \simeq \mathcal{F}\right\} .
\end{aligned}
$$

Proof. Once we show that these categories constitute a pre-s-structure, the uniqueness statement is obvious. It is clear that axioms ( 924$)$ and ( $(55)$ hold, and axiom ( $(36)$ holds by the definition of Hom. Given any $\mathcal{F} \in \mathcal{C}_{G}(U)$, let $\mathcal{F}_{1}$ be any extension of it to a coherent sheaf on $X$, and form the exact sequence

$$
0 \rightarrow \sigma_{\leq w} \mathcal{F}_{1} \rightarrow \mathcal{F}_{1} \rightarrow \sigma_{\geq w+1} \mathcal{F}_{1} \rightarrow 0
$$

Applying $j^{*}$ to this sequence gives a short exact sequence in $\mathcal{C}_{G}(U)$ showing that axiom (\$4) holds. Axiom (\$6) follows from the formula $j^{*} \mathcal{F} \otimes j^{*} \mathcal{G} \simeq j^{*}(\mathcal{F} \otimes \mathcal{G})$.

It remains to prove axiom (S1). Let $0 \rightarrow \mathcal{F}^{\prime} \rightarrow \mathcal{F} \rightarrow \mathcal{F}^{\prime \prime} \rightarrow 0$ be a short exact sequence in $\mathcal{C}_{G}(U)$. If $\mathcal{F} \in \mathcal{C}_{G}(U)_{\leq w}$, let $\mathcal{F}_{1} \in \mathcal{C}_{G}(X)_{\leq w}$ be a sheaf such that $j^{*} \mathcal{F}_{1} \simeq \mathcal{F}$. Using Lemma 2.4 and the fact that $\mathcal{C}_{G}(X)_{\leq w}$ is stable under subobjects and quotients, we find that $\mathcal{F}^{\prime}$ and $\mathcal{F}^{\prime \prime}$ lie in $\mathcal{C}_{G}(U)_{\leq w}$. Thus, $\mathcal{C}_{G}(U)_{\leq w}$ is stable under subobjects and quotients. On the other hand, suppose $\mathcal{F}^{\prime}$ and $\mathcal{F}^{\prime \prime}$ both belong to $\mathcal{C}_{G}(U)_{\leq w}$. If $\mathcal{F} \notin \mathcal{C}_{G}(U)_{\leq w}$, then from axiom (\$4), we obtain a 
nonzero morphism $\mathcal{F} \rightarrow \mathcal{G}$ with $\mathcal{G} \in \mathcal{C}_{G}(U)_{\geq w+1}$, but this is a contradiction, since $\operatorname{Hom}\left(\mathcal{F}^{\prime}, \mathcal{G}\right)=\operatorname{Hom}\left(\mathcal{F}^{\prime \prime}, \mathcal{G}\right)=0$ by axiom $(\sqrt{3})$. Thus $\mathcal{F} \in \mathcal{C}_{G}(U)_{\leq w}$, and $\mathcal{C}_{G}(U)_{\leq w}$ is stable under extensions. The same arguments show that $\mathcal{C}_{G}(U)_{\geq w}$ is stable under subobjects and extensions.

Proposition 3.6. Let $i: Z \hookrightarrow X$ be a closed $G$-invariant subscheme of $X$. Given a pre-s-structure on $X$, there is a pre-s-structure on $Z$ given by

$$
\begin{aligned}
& \mathcal{C}_{G}(Z)_{\leq w}=\left\{\mathcal{F} \mid i_{*} \mathcal{F} \in \mathcal{C}_{G}(X)_{\leq w}\right\}, \\
& \mathcal{C}_{G}(Z)_{\geq w}=\left\{\mathcal{F} \mid i_{*} \mathcal{F} \in \mathcal{C}_{G}(X)_{\geq w}\right\} .
\end{aligned}
$$

This is the unique pre-s-structure on $Z$ such that the functor $i^{*}$ is right s-exact and the functor $i^{!}$is left s-exact. Moreover, the functor $i_{*}: \mathcal{C}_{G}(Z) \rightarrow \mathcal{C}_{G}(X)$ is s-exact.

Proof. For any two sheaves $\mathcal{F}, \mathcal{G} \in \mathcal{C}_{G}(Z)$, we have

$$
\operatorname{Hom}(\mathcal{F}, \mathcal{G}) \simeq \operatorname{Hom}\left(i^{*} i_{*} \mathcal{F}, \mathcal{G}\right) \simeq \operatorname{Hom}\left(i_{*} \mathcal{F}, i_{*} \mathcal{G}\right)
$$

We also have $i_{*} \mathcal{H o m}(\mathcal{F}, \mathcal{G}) \simeq \mathcal{H} \operatorname{om}\left(i_{*} \mathcal{F}, i_{*} \mathcal{G}\right)$ and $i_{*}(\mathcal{F} \otimes \mathcal{G}) \simeq i_{*} \mathcal{F} \otimes i_{*} \mathcal{G}$. In view of these observations and the fact that $i_{*}$ is an exact functor, it is straightforward to verify all the axioms for a pre- $s$-structure. We omit the details.

If $\mathcal{F} \in \mathcal{C}_{G}(X)_{\leq w}$, then $i_{*} i^{*} \mathcal{F}$ is a quotient of $\mathcal{F}$, and hence in $\mathcal{C}_{G}(X)_{\leq w}$. Therefore, $i^{*} \mathcal{F} \in \mathcal{C}_{G}(\bar{Z})_{\leq w}$, so $i^{*}$ is right $s$-exact. A similar argument shows that $i^{!}$is left $s$-exact. The uniqueness of this $s$-structure follows from the fact that for any $\mathcal{F} \in \mathcal{C}_{G}(Z)$, we have $i^{*} i_{*} \mathcal{F} \simeq i^{!} i_{*} \mathcal{F} \simeq \mathcal{F}$. The $s$-exactness of $i_{*}$ is obvious.

Let $\kappa: Y \hookrightarrow X$ be a closed subscheme, and assume $Y$ is endowed with a pre-sstructure. We define a full subcategory of $\mathcal{C}_{G}(X)$ by

$$
\mathcal{C}_{G}^{\text {loc }}(X, Y)_{\leq w}=\left\{\mathcal{F} \in \mathcal{C}_{G}(X) \mid \kappa^{*} \mathcal{F} \in \mathcal{C}_{G}(Y)_{\leq w}\right\} .
$$

Later, in Proposition 4.2, we will see that this is a Serre subcategory of $\mathcal{C}_{G}(X)$.

Definition 3.7. Suppose $X$ is endowed with a pre-s-structure, and let

$$
\tilde{\mathcal{C}}_{G}(X)_{\geq w}=\left\{\mathcal{F} \mid \text { for some } V \Subset X,\left.\mathcal{F}\right|_{V} \in \mathcal{C}_{G}(V)_{\geq w}\right\} .
$$

The pre-s-structure is an almost s-structure if for every closed subscheme $\kappa: Y \hookrightarrow$ $X$, the induced pre-s-structure on $Y$ satisfies the following additional axioms:

(S7) $\tilde{\mathcal{C}}_{G}(Y)_{\geq w}$ is a Serre subcategory of $\mathcal{C}_{G}(Y)$.

(S8) If $\mathcal{F} \in \tilde{\mathcal{C}}_{G}(Y)_{\geq w}$ and $\mathcal{G} \in \tilde{\mathcal{C}}_{G}(Y)_{\geq v}$, then $\mathcal{F} \otimes \mathcal{G} \in \tilde{\mathcal{C}}_{G}(Y)_{\geq w+v}$.

Finally, an almost $s$-structure is an $s$-structure if the following condition also holds for every closed subscheme $\kappa: Y \hookrightarrow X$ :

(S9) If $\mathcal{F} \in \mathcal{C}_{G}^{\text {loc }}(X, Y)_{\leq w}$, then for any $r \geq 0$, there is an open subscheme $V \Subset_{Y} X$ such that $\operatorname{Ext}^{r}\left(\left.\mathcal{F}\right|_{V},\left.\kappa_{*} \mathcal{G}\right|_{V}\right)=0$ for all $\mathcal{G} \in \tilde{\mathcal{C}}_{G}(Y)_{\geq w+1}$.

It may seem at first glance that the last axiom would be quite onerous to check in specific examples. Fortunately, if $G$ acts on $X$ with finitely many orbits, this axiom follows from the others: see Theorem 10.2 ,

It is easy to see that in any pre-s-structure, $\tilde{\mathcal{C}}_{G}(Y)_{\geq w}$ is necessarily closed under subobjects and extensions, since $\mathcal{C}_{G}(V)_{\geq w}$ is for every open subscheme $V \subset Y$. Thus, axiom (S7) is equivalent to simply requiring that each $\tilde{\mathcal{C}}_{G}(Y)_{\geq w}$ be closed under quotients. 
Lemma 3.8. Let $j: U \hookrightarrow X$ be an open subscheme, and suppose $X$ has a pre-sstructure. Then

$$
\tilde{\mathcal{C}}_{G}(U)_{\geq w}=\left\{\mathcal{F} \mid \text { there exists an } \mathcal{F}_{1} \in \tilde{\mathcal{C}}_{G}(X)_{\geq w} \text { such that } j^{*} \mathcal{F}_{1} \simeq \mathcal{F}\right\} .
$$

Moreover, if $U$ is dense in $X$, then for all $\mathcal{F}_{1} \in \mathcal{C}_{G}(X)$, we have $\mathcal{F}_{1} \in \tilde{\mathcal{C}}_{G}(X)_{\geq w}$ if and only if $j^{*} \mathcal{F}_{1} \in \tilde{\mathcal{C}}_{G}(U)_{\geq w}$.

Proof. If $\mathcal{F}_{1} \in \tilde{\mathcal{C}}_{G}(X)_{\geq w}$, let $V \Subset X$ be such that $\left.\mathcal{F}_{1}\right|_{V} \in \mathcal{C}_{G}(V)_{\geq w}$. Then $V \cap U \Subset$ $U$, and clearly $\left.\left.\mathcal{F}_{1}\right|_{V \cap U} \simeq\left(j^{*} \mathcal{F}_{1}\right)\right|_{V \cap U} \in \mathcal{C}_{G}(V \cap U)_{\geq w}$, so we see that $j^{*} \mathcal{F}_{1} \in$ $\tilde{\mathcal{C}}_{G}(U)_{\geq w}$.

Next, given $\mathcal{F} \in \tilde{\mathcal{C}}_{G}(U)_{\geq w}$, let $V_{1} \Subset U$ be such that $\left.\mathcal{F}\right|_{V_{1}} \in \mathcal{C}_{G}\left(V_{1}\right)_{\geq w}$. Let $\mathcal{F}_{1}$ be any extension of $\mathcal{F}$ to a coherent sheaf on $X$ supported on $\bar{U}$. Let $V=V_{1} \cup(X \backslash \bar{U})$. Then we have $\left.\mathcal{F}_{1}\right|_{V} \in \mathcal{C}_{G}(V)_{\geq w}$, as desired, so $\tilde{\mathcal{C}}_{G}(U)_{\geq w}$ is described by (3.4).

Finally, in the case where $U$ is dense, the open subscheme $V$ of the previous paragraph coincides with $V_{1}$ and is dense in $X$, so the fact that $\left.\mathcal{F}_{1}\right|_{V} \in \mathcal{C}_{G}(V)_{\geq w}$ means that $\mathcal{F}_{1} \in \tilde{\mathcal{C}}_{G}(X)_{\geq w}$.

Lemma 3.9. Let $X$ be a scheme with an s-structure, and let $i: Z \hookrightarrow X$ be a closed subscheme. Let $\mathcal{F} \in \mathcal{D}_{G}^{\mathrm{b}}(X)$ be such that for all $k$, there is an open subscheme $V_{k} \Subset_{Z} X$ such that $\left.H^{k}(\mathcal{F})\right|_{V_{k}} \in \mathcal{C}_{G}^{\text {loc }}\left(V_{k}, V_{k} \cap Z\right)_{\leq w}$. Then, for any $r \in \mathbb{Z}$, there is a open subscheme $V \Subset_{Z} X$ with the property that $\operatorname{Hom}\left(\left.\mathcal{F}\right|_{V},\left.i_{*} \mathcal{G}[r]\right|_{V}\right)=0$ for all $\mathcal{G} \in \tilde{\mathcal{C}}_{G}(Z)_{\geq w+1}$.

Proof. Suppose $\mathcal{F} \in \mathcal{D}_{G}^{\mathrm{b}}(X)^{[a, b]}$. We proceed by induction on $b-a$. If $b-a=0$, then $\mathcal{F}$ is simply a shift of an sheaf $\mathcal{F}_{1} \in \mathcal{C}_{G}(X)$ with the property that $\left.\mathcal{F}_{1}\right|_{V_{a}} \in$ $\mathcal{C}_{G}^{\text {loc }}\left(V_{a}, V_{a} \cap Z\right)_{\leq w}$. The desired $V$ is obtained by invoking axiom (S9) on $V_{a}$.

Now, suppose $b>a$, and consider the distinguished triangle

$$
\tau^{\leq a} \mathcal{F} \rightarrow \mathcal{F} \rightarrow \tau^{\geq a+1} \mathcal{F} \rightarrow
$$

This gives rise to an exact sequence

$$
\operatorname{Hom}\left(\tau^{\geq a+1} \mathcal{F}, i_{*} \mathcal{G}[r]\right) \rightarrow \operatorname{Hom}\left(\mathcal{F}, i_{*} \mathcal{G}[r]\right) \rightarrow \operatorname{Hom}\left(\tau^{\leq a} \mathcal{F}, i_{*} \mathcal{G}[r]\right) .
$$

By the inductive assumption, the first and last terms vanish for all $\mathcal{G} \in \tilde{\mathcal{C}}_{G}(Z)_{\geq w+1}$ after restriction to suitable dense open subschemes $V^{\prime} \Subset_{Z} X$ and $V^{\prime \prime} \Subset_{Z} X$, respectively. Therefore, the middle term vanishes upon restriction to $V=V^{\prime} \cap V^{\prime \prime}$.

Lemma 3.10. Let $X$ be a scheme with an s-structure, and let $i: Z \hookrightarrow X$ and $\kappa: Y \hookrightarrow Z$ be closed subschemes. For any sheaf $\mathcal{F} \in \mathcal{C}_{G}^{\text {loc }}(X, Y)_{\leq w}$ and any $r \geq 0$, there is an open subscheme $V \Subset_{Y} Z$ such that $\left.H^{-r}\left(L i^{*} \mathcal{F}\right)\right|_{V} \in \overline{\mathcal{C}}_{G}^{\text {loc }}(V, V \cap Y)_{\leq w}$.

Proof. We prove the lemma by induction on $r$. For $r=0$, the lemma is trivial: it is immediate from the definitions that $i^{*} \mathcal{F} \in \mathcal{C}_{G}^{\text {loc }}(Z, Y)_{\leq w}$. Now, suppose $r>0$. For any $\mathcal{G} \in \tilde{\mathcal{C}}_{G}(Y)_{\geq w+1}$, we have $\operatorname{Hom}\left(L i^{*} \mathcal{F}, \kappa_{*} \mathcal{G}[r]\right) \simeq \operatorname{Hom}\left(\mathcal{F}, i_{*} \kappa_{*} \mathcal{G}[r]\right) \simeq$ $\operatorname{Ext}^{r}\left(\mathcal{F}, i_{*} \kappa_{*} \mathcal{G}\right)$. By axiom ( 99 ), there is an open subscheme $V^{\prime} \Subset_{Y} X$ such that $\operatorname{Ext}^{r}\left(\left.\mathcal{F}\right|_{V^{\prime}},\left.i_{*} \kappa_{*} \mathcal{G}\right|_{V^{\prime}}\right)=0$ for all $\mathcal{G} \in \tilde{\mathcal{C}}_{G}(Y)_{\geq w+1}$. Let $V_{1}^{\prime}=V^{\prime} \cap Z$; then $\operatorname{Hom}\left(\left.L i^{*} \mathcal{F}\right|_{V_{1}^{\prime}},\left.\kappa_{*} \mathcal{G}[r]\right|_{V_{1}^{\prime}}\right)=0$ for all $\mathcal{G} \in \tilde{\mathcal{C}}_{G}(Y)_{\geq w+1}$. In addition, we also have $\operatorname{Hom}\left(L i^{*} \mathcal{F}, \kappa_{*} \mathcal{G}[r]\right) \simeq \operatorname{Hom}\left(\tau^{\geq-r} L i^{*} \mathcal{F}, \kappa_{*} \mathcal{G}[r]\right)$, so from the distinguished triangle

$$
\tau^{[-r,-r]} L i^{*} \mathcal{F} \rightarrow \tau^{\geq-r} L i^{*} \mathcal{F} \rightarrow \tau^{\geq-r+1} L i^{*} \mathcal{F} \rightarrow
$$


we obtain the exact sequence

$$
\begin{aligned}
\cdots \rightarrow \operatorname{Hom}\left(\tau^{\geq-r} L i^{*} \mathcal{F}, \kappa_{*} \mathcal{G}[r]\right) \rightarrow & \operatorname{Hom}\left(\tau^{[-r,-r]} L i^{*} \mathcal{F}, \kappa_{*} \mathcal{G}[r]\right) \rightarrow \\
& \operatorname{Hom}\left(\tau^{\geq-r+1} L i^{*} \mathcal{F}[-1], \kappa_{*} \mathcal{G}[r]\right) \rightarrow \cdots .
\end{aligned}
$$

Note that $\tau^{[-r,-r]} L i^{*} \mathcal{F} \simeq H^{-r}\left(L i^{*} \mathcal{F}\right)[r]$. The sequence above can be rewritten as

$$
\operatorname{Hom}\left(L i^{*} \mathcal{F}, \kappa_{*} \mathcal{G}[r]\right) \rightarrow \operatorname{Hom}\left(H^{-r}\left(L i^{*} \mathcal{F}\right), \kappa_{*} \mathcal{G}\right) \rightarrow \operatorname{Hom}\left(\tau^{\geq-(r-1)} L i^{*} \mathcal{F}, \kappa_{*} \mathcal{G}[r+1]\right)
$$

The first term vanishes when we restrict to $V_{1}^{\prime}$. By the inductive assumption, all cohomology sheaves of $\tau^{\geq-(r-1)} L i^{*} \mathcal{F}$ satisfy the hypothesis of Lemma 3.9, so invoking that lemma, we see that the last term above vanishes upon restriction to some dense open subscheme $V^{\prime \prime} \Subset_{Y} Z$. Then, clearly, the middle term vanishes upon restriction to $V=V_{1}^{\prime} \cap V^{\prime \prime}$. Let $\tilde{\kappa}: V \cap Y \hookrightarrow V$ denote the inclusion map. The vanishing of the middle term above implies, in particular, that

$$
\operatorname{Hom}\left(\left.H^{-r}\left(L i^{*} \mathcal{F}\right)\right|_{V}, \tilde{\kappa}_{*} \mathcal{G}_{1}\right)=\operatorname{Hom}\left(\left.\tilde{\kappa}^{*} H^{-r}\left(L i^{*} \mathcal{F}\right)\right|_{V}, \mathcal{G}_{1}\right)=0
$$

for all $\mathcal{G}_{1} \in \mathcal{C}_{G}(V \cap Y)_{\geq w+1}$, from which it follows in turn that $\left.\tilde{\kappa}^{*} H^{-r}\left(L i^{*} \mathcal{F}\right)\right|_{V} \in$ $\mathcal{C}_{G}(V \cap Y)_{\leq w}$. Thus, $\left.H^{-r}\left(L i^{*} \mathcal{F}\right)\right|_{V} \in \mathcal{C}_{G}^{\text {loc }}(V, V \cap Y)_{\leq w}$, as desired.

Lemma 3.11. Let $X$ be a scheme with an s-structure. The induced pre-s-structure on any open or closed subscheme is an s-structure.

Proof. Let $U \subset X$ be an open subscheme, and let $Y \subset U$ be a closed subscheme. It is straightforward to deduce the conditions in axioms (S7)-(S9) from the corresponding facts for the closed subscheme $\bar{Y} \subset X$. We omit the details.

Now, let $i: Z \hookrightarrow X$ be a closed subscheme. Axioms (S7) and (\$8) hold for $Z$ automatically, since any closed subscheme of $Z$ is also a closed subscheme of $X$.

We now treat axiom (S9). Let $\kappa: Y \hookrightarrow Z$ be a closed subscheme, and let $\mathcal{F} \in \mathcal{C}_{G}^{\text {loc }}(Z, Y)_{\leq w}$. Fix an integer $r \geq 0$. By the same axiom for $X$, we know that there exists an open subscheme $V_{0} \Subset_{Y} X$ such that for all $\mathcal{G} \in \tilde{\mathcal{C}}_{G}(Y)_{\geq w+1}$, $\mathbf{E x t}^{r}\left(\left.i_{*} \mathcal{F}\right|_{V_{0}},\left.i_{*} \kappa_{*} \mathcal{G}\right|_{V_{0}}\right)=0$. We assume inductively that (\$9) is already known for $\mathbf{E x t}^{k}$-groups with $k<r$. For each $k$ with $1 \leq k \leq r-1$, let us invoke Lemma 3.10 to find an open subset $U_{k} \Subset_{Y} Z$ such that $\left.H^{-k}\left(L i^{*} i_{*} \mathcal{F}\right)\right|_{U_{k}} \in \mathcal{C}_{G}^{\text {loc }}\left(U_{k}, U_{k} \cap Y\right)_{\leq w}$. Then, the inductive assumption gives us an open set $V_{k} \Subset_{U_{k} \cap Y} U_{k}$ such that $\mathbf{E x t}^{r-k-1}\left(\left.H^{-k}\left(L i^{*} i_{*} \mathcal{F}\right)\right|_{V_{k}},\left.\kappa_{*} \mathcal{G}\right|_{V_{k}}\right)=0$ for all $\mathcal{G} \in \tilde{\mathcal{C}}_{G}(Y)_{\geq w+1}$. Note that $V_{k} \Subset_{Y}$ $Z$, and let

$$
V=V_{0} \cap V_{1} \cap \cdots \cap V_{k}
$$

Clearly, $V \Subset_{Y} Z$, and all the Ext-vanishing statements above hold over $V$. In particular, for $k=1, \ldots, r-1$, the collection of statements

$$
\operatorname{Ext}^{r-k-1}\left(\left.H^{-k}\left(L i^{*} i_{*} \mathcal{F}\right)\right|_{V},\left.\kappa_{*} \mathcal{G}\right|_{V}\right) \simeq \operatorname{Hom}\left(\left.H^{-k}\left(L i^{*} i_{*} \mathcal{F}\right)\right|_{V}[k+1],\left.\kappa_{*} \mathcal{G}\right|_{V}[r]\right)=0
$$

lets us deduce (via a standard argument by induction on the number of nonzero $\left.\left.H^{-k}\left(L i^{*} i_{*} \mathcal{F}\right)\right|_{V}\right)$ that $\operatorname{Hom}\left(\left(\left.\tau^{[-r+1,-1]} L i^{*} i_{*} \mathcal{F}\right|_{V}\right)[1],\left.\kappa_{*} \mathcal{G}\right|_{V}[r]\right)=0$. Since we have $\left.\kappa_{*} \mathcal{G}\right|_{V}[r] \in \mathcal{D}_{G}^{\mathrm{b}}(V)^{\geq-r}$, it follows that

$$
\operatorname{Hom}\left(\left(\left.\tau^{\leq-1} L i^{*} i_{*} \mathcal{F}\right|_{V}\right)[1],\left.\kappa_{*} \mathcal{G}\right|_{V}[r]\right)=0 .
$$

Next, we know that $\operatorname{Ext}^{r}\left(\left.i_{*} \mathcal{F}\right|_{V_{0}},\left.i_{*} \kappa_{*} \mathcal{G}\right|_{V_{0}}\right) \simeq \operatorname{Hom}\left(\left.L i^{*} i_{*} \mathcal{F}\right|_{V_{0} \cap Z},\left.\kappa_{*} \mathcal{G}\right|_{V_{0} \cap Z}[r]\right)=$ 0 , so

$$
\operatorname{Hom}\left(\left.L i^{*} i_{*} \mathcal{F}\right|_{V},\left.\kappa_{*} \mathcal{G}\right|_{V}[r]\right)=0
$$


as well. Note that $\left.\left.\left.\tau^{\geq 0} L i^{*} i_{*} \mathcal{F}\right|_{V} \simeq i^{*} i_{*} \mathcal{F}\right|_{V} \simeq \mathcal{F}\right|_{V}$, so we have a distinguished triangle

$$
\left.\left.\left.\tau^{\leq-1} L i^{*} i_{*} \mathcal{F}\right|_{V} \rightarrow L i^{*} i_{*} \mathcal{F}\right|_{V} \rightarrow \mathcal{F}\right|_{V} \rightarrow .
$$

From the long exact sequence of Hom-groups associated to this distinguished triangle and the two vanishing statements established above, we see that

$$
\operatorname{Hom}\left(\left.\mathcal{F}\right|_{V},\left.\kappa_{*} \mathcal{G}\right|_{V}[r]\right) \simeq \operatorname{Ext}^{r}\left(\left.\mathcal{F}\right|_{V},\left.\kappa_{*} \mathcal{G}\right|_{V}\right)=0
$$

for any $\mathcal{G} \in \tilde{\mathcal{C}}_{G}(Y)_{\geq w+1}$, as desired.

We conclude with a basic fact about the structure sheaf of a scheme with an $s$-structure.

Proposition 3.12. If $X$ is endowed with an s-structure, then $\mathcal{O}_{X} \in \mathcal{C}_{G}(X)_{\leq 0}$, but $\mathcal{O}_{X} \notin \mathcal{C}_{G}(X)_{\leq-1}$. If $X$ is reduced, then $\mathcal{O}_{X}$ is pure of step 0 .

Proof. By axiom ( $(55)$, there must be some integer $v$ such that $\mathcal{O}_{X} \in \mathcal{C}_{G}(X)_{\leq v}$. It follows that $\left.\mathcal{O}_{X}\right|_{U} \in \mathcal{C}_{G}(U)_{\leq v}$ for every open subscheme $U \subset X$. Now, suppose that $\mathcal{O}_{X} \in \mathcal{C}_{G}(X)_{\geq 1}$. Then $v \geq 1$, so by axiom (\$8), we have that $\mathcal{O}_{X}^{\otimes v+1} \in \tilde{\mathcal{C}}_{G}(X)_{\geq v+1}$, so there is some dense open subscheme $U$ with $\left.\mathcal{O}_{X}^{\otimes v+1}\right|_{U} \in \mathcal{C}_{G}(U)_{\geq v+1}$. But of course $\mathcal{O}_{X}^{\otimes v+1} \simeq \mathcal{O}_{X}$, so this is a contradiction, and $\mathcal{O}_{X} \notin \mathcal{C}_{G}(X)_{\geq 1}$.

Next, consider $\sigma_{\geq 1} \mathcal{O}_{X}$. This is a quotient of $\mathcal{O}_{X}$, and thus isomorphic to $i_{*} \mathcal{O}_{Z}$ for some closed subscheme $i: Z \hookrightarrow X$. Since $i_{*}$ is $s$-exact, we must have $\mathcal{O}_{Z} \in \mathcal{C}_{G}(Z)_{\geq 1}$, but this is impossible by the previous paragraph, so it follows that $\sigma_{\geq 1} \mathcal{O}_{X}=0$. Thus, $\mathcal{O}_{X} \in \mathcal{C}_{G}(X)_{\leq 0}$.

Now, let $v$ be the smallest integer such that $\mathcal{O}_{X} \in \mathcal{C}_{G}(X)_{\leq v}$. We have just seen that $v \leq 0$. On the other hand, we cannot have $v<0$, for in that case, axiom (S6) would give us that

$$
\mathcal{O}_{X} \simeq \mathcal{O}_{X} \otimes \mathcal{O}_{X} \in \mathcal{C}_{G}(X)_{\leq 2 v}
$$

So $v=0$, and $\mathcal{O}_{X} \notin \mathcal{C}_{G}(X)_{\leq-1}$.

Finally, consider the subsheaf $\sigma_{\leq-1} \mathcal{O}_{X} \subset \mathcal{O}_{X}$. Over any open subscheme $U \subset X$, the restriction $\left.\sigma_{\leq-1} \mathcal{O}_{X}\right|_{U}$ is a proper subsheaf of $\mathcal{O}_{U}$, since (by the above argument) $\mathcal{O}_{U} \notin \mathcal{C}_{G}(U)_{\leq-1}$. This condition implies that $\sigma_{\leq-1} \mathcal{O}_{X}$ is a nilpotent ideal sheaf. In particular, if $X$ is reduced, $\sigma_{\leq-1} \mathcal{O}_{X}=0$.

\section{4. $s$-Structures on Closed Subschemes}

Throughout this section, we fix the following conventions: let

$$
i: Z \hookrightarrow X
$$

denote the inclusion of a closed subscheme, and let $\mathcal{I}_{Z} \subset \mathcal{O}_{X}$ denote the corresponding ideal sheaf. We assume that $Z$ is endowed with a pre- $s$-structure. (We do not assume that $X$ has a pre-s-structure.) In addition, we impose the assumption (cf. axiom (A1) in Definition 5.1) that

$$
i^{*} \mathcal{I}_{Z} \in \mathcal{C}_{G}(Z)_{\leq 0} .
$$

We will frequently consider a second closed subscheme $Z^{\prime} \subset X$ with the same underlying topological space as $Z$, such that there is an inclusion map

$$
t: Z \hookrightarrow Z^{\prime} .
$$

In this setting, we let $\mathcal{I} \subset \mathcal{O}_{Z^{\prime}}$ denote the ideal sheaf of $Z \subset Z^{\prime}$. Note that $t^{*} \mathcal{I}$ is naturally a quotient of $i^{*} \mathcal{I}_{Z}$, so it follows from (4.1) that $t^{*} \mathcal{I} \in \mathcal{C}_{G}(Z)_{\leq 0}$ as well. 
One goal of this section is to show that the given pre-s-structure on $Z$ induces pre-s-structures on all such "larger" subschemes $Z^{\prime}$. This allows us to define a full subcategory of $\mathcal{C}_{G}(X)$ as follows:

$$
\mathcal{C}_{G}^{\text {supp }}(X, Z)_{\geq w}=\left\{\mathcal{F} \mid \begin{array}{l}
\text { there is a subscheme structure } i^{\prime}: Z^{\prime} \hookrightarrow X \text { on } \underline{Z} \\
\text { and a sheaf } \mathcal{F}_{1} \in \mathcal{C}_{G}\left(Z^{\prime}\right)_{\geq w} \text { such that } \mathcal{F} \simeq i_{*}^{\prime} \mathcal{F}_{1}
\end{array}\right\}
$$

Recall also the definition of $\mathcal{C}_{G}^{\text {loc }}(X, Z)_{\leq w}$ from (3.3). Another result of this section is that $\mathcal{C}_{G}^{\text {loc }}(X, Z)_{\leq w}$ is a Serre subcategory of $\mathcal{C}_{G}(X)$. These two categories play important and complementary roles in the gluing theorem of Section 5. Indeed, in the special case where $X$ is simply another scheme structure on $\underline{Z}$, most of the gluing theorem is contained in the results of this section.

Proposition 4.1. There is a unique pre-s-structure on $Z^{\prime}$ such that $t_{*}$ is s-exact. It is given by:

$$
\begin{aligned}
& \mathcal{C}_{G}\left(Z^{\prime}\right)_{\leq w}=\left\{\mathcal{F} \mid t^{*} \mathcal{F} \in \mathcal{C}_{G}(Z)_{\leq w}\right\}, \\
& \mathcal{C}_{G}\left(Z^{\prime}\right)_{\geq w}=\left\{\mathcal{G} \mid \operatorname{Hom}(\mathcal{F}, \mathcal{G})=0 \text { for all } \mathcal{F} \in \mathcal{C}_{G}\left(Z^{\prime}\right)_{\leq w}\right\} .
\end{aligned}
$$

Moreover, $t^{*}$ is right $s$-exact, and $t^{!}$is left $s$-exact.

Proof. We begin by proving that $\mathcal{C}_{G}\left(Z^{\prime}\right) \leq w$ is a Serre subcategory of $\mathcal{C}_{G}\left(Z^{\prime}\right)$. It is obviously stable under extensions and quotients, because $\mathcal{C}_{G}(Z)_{\leq w}$ is, and $t^{*}$ is a right exact functor. It remains to show that it is closed under subobjects.

Note that $\mathcal{I} \subset \mathcal{O}_{Z^{\prime}}$ is a nilpotent ideal sheaf, so for any $\mathcal{F} \in \mathcal{C}_{G}\left(Z^{\prime}\right)$, there is an integer $n$ such that $\mathcal{I}^{n} \mathcal{F}=0$. Let $\ell(\mathcal{F})$ be the smallest such integer. Now, suppose $\mathcal{F} \in \mathcal{C}_{G}\left(Z^{\prime}\right)_{\leq w}$. We will prove by induction on $\ell(\mathcal{F})$ that all subsheaves of $\mathcal{F}$ are also in $\mathcal{C}_{G}\left(Z^{\prime}\right)_{\leq w}$. If $\ell(\mathcal{F}) \leq 1$, then $\mathcal{F}$ is already supported on $Z$ : i.e., $\mathcal{F} \simeq t_{*} \mathcal{F}_{1}$ for some $\mathcal{F}_{1} \in \mathcal{C}_{G}(Z)$. Moreover, we necessarily have $\mathcal{F}_{1} \in \mathcal{C}_{G}(Z)_{\leq w}$, since $t^{*} \mathcal{F} \simeq \mathcal{F}_{1}$. Every subsheaf of $\mathcal{F}$ is in $\mathcal{C}_{G}\left(Z^{\prime}\right)_{\leq w}$ because every subsheaf of $\mathcal{F}_{1}$ is in $\mathcal{C}_{G}(Z)_{\leq w}$.

Next, if $\ell(\mathcal{F})>1$, form the short exact sequence

$$
0 \rightarrow \mathcal{I F} \rightarrow \mathcal{F} \rightarrow \mathcal{F} / \mathcal{I F} \rightarrow 0
$$

It is clear that $\ell(\mathcal{I} \mathcal{F})=\ell(\mathcal{F})-1$, and that $\ell(\mathcal{F} / \mathcal{I} \mathcal{F})=1$. Note that $\mathcal{F} / \mathcal{I} \mathcal{F} \simeq t_{*} t^{*} \mathcal{F}$ lies in $\mathcal{C}_{G}\left(Z^{\prime}\right)_{\leq w}$ by assumption. On the other hand, $\mathcal{I} \mathcal{F}$ is a quotient of $\mathcal{I} \otimes \mathcal{F}$, and we have $t^{*}(\mathcal{I} \otimes \mathcal{F}) \simeq t^{*} \mathcal{I} \otimes t^{*} \mathcal{F} \in \mathcal{C}_{G}(Z)_{\leq w}$ by condition (4.1). Any subsheaf of $\mathcal{F}$ is an extension of a subsheaf of $\mathcal{F} / \mathcal{I} \mathcal{F}$ by a subsheaf of $\mathcal{I} \mathcal{F}$. The latter two are in $\mathcal{C}_{G}\left(Z^{\prime}\right)_{\leq w}$, and since we already know that $\mathcal{C}_{G}\left(Z^{\prime}\right)_{\leq w}$ is stable under extensions, we conclude that any subsheaf of $\mathcal{F}$ is in $\mathcal{C}_{G}\left(Z^{\prime}\right)_{\leq w}$. It is clear that $\mathcal{C}_{G}\left(Z^{\prime}\right)_{\geq w}$ is stable under subobjects and extensions, so axiom (S1) holds. We have $\mathcal{C}_{G}\left(Z^{\prime}\right)_{\geq w}=\mathcal{C}_{G}\left(Z^{\prime}\right)_{\leq w}^{\perp}$ by definition, so by Proposition 2.7, axiom (\$4) holds. Axiom (S2) is obvious.

The idea of the induction argument above will be reused several times. The categories $\mathcal{C}_{G}\left(Z^{\prime}\right)$ and $\mathcal{C}_{G}\left(Z^{\prime}\right)_{\leq w}$ (and later, in Proposition 4.5, $\tilde{\mathcal{C}}_{G}\left(Z^{\prime}\right)_{\geq w}$ as well) are Serre subcategories of $\mathcal{C}_{G}\left(Z^{\prime}\right)$, so given a sheaf $\mathcal{F}$ in any of these categories, we can always form the short exact sequence (4.3), and the sheaves $\mathcal{I F}$ and $\mathcal{F} / \mathcal{I} \mathcal{F}$ both lie in the same category. Given any property that is stable under extensions, we can prove that it holds for all sheaves in $\mathcal{C}_{G}\left(Z^{\prime}\right)\left(\operatorname{resp} . \mathcal{C}_{G}\left(Z^{\prime}\right)_{\leq w}, \tilde{\mathcal{C}}_{G}\left(Z^{\prime}\right)_{\geq w}\right)$ simply by proving it for sheaves with $\ell(\mathcal{F})=1$, i.e., for sheaves of the form $\mathcal{F}=t_{*} \mathcal{F}_{1}$ with $\mathcal{F}_{1} \in \mathcal{C}_{G}(Z)$. (Note that this method cannot be used to prove statements about $\mathcal{C}_{G}\left(Z^{\prime}\right)_{\geq w}$, because for $\mathcal{F} \in \mathcal{C}_{G}\left(Z^{\prime}\right)_{\geq w}$, it may not be true that $\mathcal{F} / \mathcal{I} \mathcal{F} \in \mathcal{C}_{G}\left(Z^{\prime}\right)_{\geq w}$.) 
Now, fix a sheaf $\mathcal{G} \in \mathcal{C}_{G}\left(Z^{\prime}\right)_{\geq w}$. To show that $\operatorname{Hom}(\mathcal{F}, \mathcal{G})=0$ for all $\mathcal{F} \in$ $\mathcal{C}_{G}\left(Z^{\prime}\right)_{\leq w}$, it suffices to show, by the previous paragraph, that $\operatorname{Hom}\left(t_{*} \mathcal{F}_{1}, \mathcal{G}\right)=0$ for all $\mathcal{F}_{1} \in \mathcal{C}_{G}(Z)_{\leq w}$. Since $\operatorname{Hom}\left(t_{*} \mathcal{F}_{1}, \mathcal{G}\right) \simeq \operatorname{Hom}\left(\mathcal{F}_{1}, t^{\prime} \mathcal{G}\right)=0$ for all $\mathcal{F}_{1} \in$ $\mathcal{C}_{G}(Z)_{\leq w}$, we see that $t^{\prime} \mathcal{G} \in \mathcal{C}_{G}(Z)_{\geq w}$. Now, for any open subscheme $V \subset Z^{\prime}$, we have $\operatorname{Hom}\left(\left.\left(t_{*} \mathcal{F}_{1}\right)\right|_{V},\left.\mathcal{G}\right|_{V}\right) \simeq \operatorname{Hom}\left(\left.\mathcal{F}_{1}\right|_{V_{1} \cap Z},\left.\left(t^{!} \mathcal{G}\right)\right|_{V_{1} \cap Z}\right)$, and the latter is 0 because $\operatorname{Hom}\left(\mathcal{F}_{1}, t^{\prime} \mathcal{G}\right)=0$. Therefore, $\operatorname{Hom}\left(t_{*} \mathcal{F}_{1}, \mathcal{G}\right)=0$, and axiom (A3) holds.

Given $\mathcal{G} \in \mathcal{C}_{G}\left(Z^{\prime}\right)$, consider the sheaf $t^{!} \mathcal{G} \in \mathcal{C}_{G}(Z)$. By axiom (\$5) for $Z$, we have $t^{!} \mathcal{G} \in \mathcal{C}_{G}(Z)_{>w}$ for some $w$, so $\operatorname{Hom}\left(\mathcal{F}_{1}, t^{!} \mathcal{G}\right)=0$ for all $\mathcal{F}_{1} \in \mathcal{C}_{G}(Z)_{<w-1}$. It follows that $\operatorname{Hom}\left(t_{*} \mathcal{F}_{1}, \mathcal{G}\right)=0$, and then, by induction on $\ell(\mathcal{F})$, that $\operatorname{Hom}(\mathcal{F}, \mathcal{G})=0$ for all $\mathcal{F} \in \mathcal{C}_{G}\left(Z^{\prime}\right)_{\leq w-1}$. Therefore, $\mathcal{G} \in \mathcal{C}_{G}\left(Z^{\prime}\right)_{\geq w}$. On the other hand, there is some $v \in \mathbb{Z}$ such that $t^{*} \mathcal{G} \in \mathcal{C}_{G}(Z)_{\leq v}$, and it follows that $\mathcal{G} \in \mathcal{C}_{G}\left(Z^{\prime}\right)_{\leq v}$. Thus, axiom ( $(55)$ holds for $Z^{\prime}$.

Next, fix a sheaf $\mathcal{G} \in \mathcal{C}_{G}\left(Z^{\prime}\right)_{\leq v}$. To verify axiom (\$6), it suffices to show that $t_{*} \mathcal{F}_{1} \otimes \mathcal{G} \in \mathcal{C}_{G}\left(Z^{\prime}\right)_{\leq w+v}$ for all $\mathcal{F}^{\prime} \in \mathcal{C}_{G}(Z)_{\leq w}$. This is straightforward: we have $t_{*} \mathcal{F}_{1} \otimes \mathcal{G} \simeq t_{*}\left(\mathcal{F}_{1} \otimes t^{*} \mathcal{G}\right)$, and since $t^{*} \mathcal{G} \in \mathcal{C}_{G}(Z)_{\leq v}$, we know that $\mathcal{F}^{\prime} \otimes t^{*} \mathcal{G} \in$ $\mathcal{C}_{G}(Z)_{\leq w+v}$. This completes the proof that the categories above constitute a pre$s$-structure.

We saw earlier that $\mathcal{G} \in \mathcal{C}_{G}\left(Z^{\prime}\right)_{\geq w}$ implies $t^{!} \mathcal{G} \in \mathcal{C}_{G}(Z)_{\geq w}$, so $t^{!}$is left $s$-exact. It is obvious, from the definition of $\mathcal{C}_{G}\left(Z^{\prime}\right)_{\leq w}$, that $t^{*}$ is right $s$-exact. Finally, we consider the uniqueness statement: suppose we had another collection of subcategories $\left(\left\{\mathcal{C}_{\leq w}^{\prime}\right\},\left\{\mathcal{C}_{\geq w}^{\prime}\right\}\right)$ that formed a pre-s-structure and with respect to which $t_{*}$ was $s$-exact. For $\mathcal{F} \in \mathcal{C}_{G}\left(Z^{\prime}\right)_{\leq w}$, one sees by induction an $\ell(\mathcal{F})$ that $\mathcal{F} \in \mathcal{C}_{\leq w}^{\prime}$, so $\mathcal{C}_{G}\left(Z^{\prime}\right)_{\leq w} \subset \mathcal{C}_{\leq w}^{\prime}$. But since $\mathcal{C}_{\leq w}^{\prime}$ is itself a Serre subcategory of $\mathcal{C}_{G}\left(Z^{\prime}\right)$, the same argument goes through with the roles of $\mathcal{C}_{G}\left(Z^{\prime}\right)_{\leq w}$ and $\mathcal{C}_{\leq w}^{\prime}$ reversed: we thus obtain $\mathcal{C}_{\leq w}^{\prime} \subset \mathcal{C}_{G}\left(Z^{\prime}\right)_{\leq w}$. Thus, $\mathcal{C}_{\leq w}^{\prime}=\mathcal{C}_{G}\left(Z^{\prime}\right)_{\leq w}$, and then the equality $\mathcal{C}_{\geq w}^{\prime}=\mathcal{C}_{G}\left(Z^{\prime}\right)_{\geq w}$ follows from the fact that $\mathcal{C}_{\geq w}^{\prime}=\mathcal{C}_{\leq w-1}^{\prime} \perp$ and $\mathcal{C}_{G}\left(Z^{\prime}\right)_{\geq w}=\mathcal{C}_{G}\left(Z^{\prime}\right)_{\leq w-1}^{\perp}$.

The uniqueness statement in Proposition 4.1 implies that the condition in the definition of $\mathcal{C}_{G}^{\mathrm{supp}}(X, Z)_{\geq w}$ may be tested on any subscheme structure on which a given sheaf is supported. That is, given $\mathcal{F} \in \mathcal{C}_{G}(X)$, suppose we have two subscheme structures $i^{\prime}: Z^{\prime} \hookrightarrow X$ and $i^{\prime \prime}: Z^{\prime \prime} \hookrightarrow X$ on $\underline{Z}$, and sheaves $\mathcal{F}^{\prime} \in \mathcal{C}_{G}\left(Z^{\prime}\right)$, $\mathcal{F}^{\prime \prime} \in \mathcal{C}_{G}\left(Z^{\prime \prime}\right)$ such that $\mathcal{F} \simeq i_{*}^{\prime} \mathcal{F}^{\prime} \simeq i_{*}^{\prime \prime} \mathcal{F}^{\prime \prime}$. Let $Y=Z^{\prime} \cup Z^{\prime \prime}$ (that is, if $Z^{\prime}$ and $Z^{\prime \prime}$ correspond to ideal sheaves $\mathcal{I}^{\prime}$ and $\mathcal{I}^{\prime \prime}$, respectively, then $Y$ is the subscheme structure on $\underline{Z}$ corresponding to $\mathcal{I}^{\prime} \cap \mathcal{I}^{\prime \prime}$ ). Then the pre-s-structure on $Y$ induced from $Z^{\prime}$ coincides with that induced from $Z^{\prime \prime}$, because both of them must agree with the one induced from $Z$. By comparing the push-forwards of $\mathcal{F}^{\prime}$ and $\mathcal{F}^{\prime \prime}$ to $Y$, we see that $\mathcal{F}^{\prime} \in \mathcal{C}_{G}\left(Z^{\prime}\right)_{\geq w}$ if and only if $\mathcal{F}^{\prime \prime} \in \mathcal{C}_{G}\left(Z^{\prime \prime}\right)_{\geq w}$.

Proposition 4.2. For each $w \in \mathbb{Z}, \mathcal{C}_{G}^{\text {loc }}(X, Z)_{\leq w}$ is a Serre subcategory of $\mathcal{C}_{G}(X)$.

Proof. Because $i^{*}$ is a right exact functor, and $\mathcal{C}_{G}(Z)_{\leq w}$ is closed under quotients and extensions, it is clear that $\mathcal{C}_{G}^{\text {loc }}(X, Z)_{\leq w}$ is closed under quotients and extensions as well. It remains to show that it is closed under subobjects. Note first that for any $k \geq 1$, the sheaf $\mathcal{F} / \mathcal{I}^{k} \mathcal{F}$ is annihilated by some power of $\mathcal{I}$, and therefore supported on some subscheme structure $i_{Z^{\prime}}: Z^{\prime} \hookrightarrow X$ on $\underline{Z}$. Indeed, if $\mathcal{F} / \mathcal{I}^{k} \mathcal{F} \simeq i_{Z^{\prime} *} \mathcal{F}_{1}$, then we must have $\mathcal{F}_{1} \in \mathcal{C}_{G}\left(Z^{\prime}\right)_{\leq w}$. We know that every subsheaf of $\mathcal{F}_{1}$ lies in $\mathcal{C}_{G}\left(Z^{\prime}\right)_{\leq w}$. It follows that every subsheaf of $\mathcal{F} / \mathcal{I}^{k} \mathcal{F}$ lies in $\mathcal{C}_{G}^{\text {loc }}(X, Z)_{\leq w}$.

Now, consider a subsheaf $\mathcal{F}^{\prime} \subset \mathcal{F}$. Let $\mathcal{F}^{\prime \prime}=\mathcal{F}^{\prime} / \mathcal{I} \mathcal{F}^{\prime} \simeq i_{*} i^{*} \mathcal{F}^{\prime}$. Clearly, $i^{*} \mathcal{F}^{\prime \prime} \simeq$ $i^{*} \mathcal{F}^{\prime}$, so it suffices to show that $\mathcal{F}^{\prime \prime} \in \mathcal{C}_{G}^{\text {loc }}(X, Z)_{\leq w}$. By the Artin-Rees lemma for coherent sheaves on a noetherian scheme, there exists an integer $k \geq 1$ such that 
for all $n \geq k$, we have

$$
\mathcal{I}^{n} \mathcal{F} \cap \mathcal{F}^{\prime}=\mathcal{I}^{n-k}\left(\mathcal{I}^{k} \mathcal{F} \cap \mathcal{F}^{\prime}\right) .
$$

Let us take $n=k+1$. Obviously

$$
\mathcal{I}\left(\mathcal{I}^{k} \mathcal{F} \cap \mathcal{F}^{\prime}\right) \subset \mathcal{I F}^{\prime} \subset \mathcal{F}^{\prime},
$$

so $\mathcal{F}^{\prime} / \mathcal{I} \mathcal{F}^{\prime}$ is a quotient of the sheaf $\mathcal{G}=\mathcal{F}^{\prime} /\left(\mathcal{I}\left(\mathcal{I}^{k} \mathcal{F} \cap \mathcal{F}^{\prime}\right)\right)$. But then $\mathcal{G} \simeq$ $\mathcal{F}^{\prime} /\left(\mathcal{I}^{k+1} \mathcal{F} \cap \mathcal{F}^{\prime}\right)$, so $\mathcal{G}$ can be identified with a subsheaf of $\mathcal{F} / \mathcal{I}^{k+1} \mathcal{F}$. By the previous paragraph, $\mathcal{G} \in \mathcal{C}_{G}^{\text {loc }}(X, Z)_{\leq w}$. Since $\mathcal{C}_{G}^{\text {loc }}(X, Z)_{\leq w}$ is closed under quotients, we see that $\mathcal{F}^{\prime \prime} \in \mathcal{C}_{G}^{\text {loc }}(X, Z)_{\leq w}$ as well.

For the remainder of the section, we study almost $s$-structures that obey an additional condition:

Definition 4.3. An almost $s$-structure on $X$ is said to be a weak $s$-structure if for every $\mathcal{F} \in \mathcal{C}_{G}(X)_{\leq w}$ and every $\mathcal{G} \in \tilde{\mathcal{C}}_{G}(X)_{\geq w+1}$, there is an open subscheme $V \Subset X$ such that $\operatorname{Ext}^{1}\left(\left.\mathcal{F}\right|_{V},\left.\mathcal{G}\right|_{V}\right)=0$.

Obviously, this condition is a special case of axiom ( 99 ), so every $s$-structure is a weak $s$-structure. However, it turns out that weak $s$-structures are easier to study in the context of the construction of Proposition 4.1. In Section 5 , additional hypotheses will allow us to obtain $s$-structures, but we will require the following results on weak $s$-structures along the way.

Lemma 4.4. Assume that $Z$ is endowed with a weak s-structure. Then, for any $\mathcal{F} \in \mathcal{C}_{G}(Z)_{\leq w}$ and any $\mathcal{G} \in \tilde{\mathcal{C}}_{G}(Z)_{\geq w+1}$, there is an open subscheme $V \Subset Z^{\prime}$ such that $\mathbf{E x t}^{1}\left(\left.t_{*} \mathcal{F}\right|_{V},\left.t_{*} \mathcal{G}\right|_{V}\right)=0$.

Proof. From Definition 4.3, there is a dense open subscheme $V_{1} \Subset Z$ such that $\operatorname{Ext}^{1}\left(\left.\mathcal{F}\right|_{V_{1}},\left.\mathcal{G}\right|_{V_{1}}\right)=0$. By replacing $V_{1}$ by a smaller open subscheme if necessary, we may assume that $\left.\mathcal{G}\right|_{V_{1}} \in \mathcal{C}_{G}\left(V_{1}\right)_{\geq w+1}$. Let $V$ be the corresponding open subscheme of $Z^{\prime}$.

We will show that any short exact sequence $\left.\left.0 \rightarrow t_{*} \mathcal{G}\right|_{V} \rightarrow \mathcal{H} \rightarrow t_{*} \mathcal{F}\right|_{V} \rightarrow 0$ in $\mathcal{C}_{G}(V)$ splits. The same argument will also be valid when $V$ is replaced by any open subscheme of $V$, so it will follow that $\operatorname{Ext}^{1}\left(\left.t_{*} \mathcal{F}\right|_{V},\left.t_{*} \mathcal{G}\right|_{V}\right)=0$.

For brevity, we will write $\mathcal{I}$ instead of $\left.\mathcal{I}\right|_{V}$ for the ideal sheaf in $\mathcal{O}_{V}$ corresponding to the closed subscheme $V_{1}=V \cap Z$. Let us identify $\left.t_{*} \mathcal{G}\right|_{V}$ with its image in $\mathcal{H}$. Because $\left.t_{*} \mathcal{F}\right|_{V}$ is supported on $V_{1}$, we see that $\mathcal{I} \mathcal{H}$ must be contained in the kernel of the morphism $\left.\mathcal{H} \rightarrow t_{*} \mathcal{F}\right|_{V}$; i.e., $\left.\mathcal{I} \mathcal{H} \subset t_{*} \mathcal{G}\right|_{V}$. In particular, we see that $\mathcal{I H} \in \mathcal{C}_{G}(V)_{\geq w+1}$. Consider the diagram

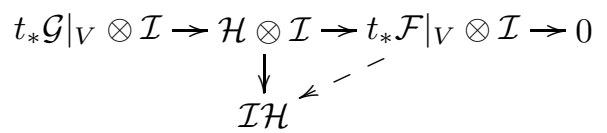

The top row is right exact. Since $\left.t_{*} \mathcal{G}\right|_{V}$ is supported on $V_{1}$, it is annihilated by $\mathcal{I}$, so the natural map $\left.t_{*} \mathcal{G}\right|_{V} \otimes \mathcal{I} \rightarrow \mathcal{I H}$ is 0 . In other words, the image of $\left.t_{*} \mathcal{G}\right|_{V} \otimes \mathcal{I}$ in $\mathcal{H} \otimes \mathcal{I}$ is contained in the kernel of the map $\mathcal{H} \otimes \mathcal{I} \rightarrow \mathcal{I H}$, so the latter factors through $\left.t_{*} \mathcal{F}\right|_{V} \otimes \mathcal{I}$. Now, it is obvious from the definition of $\mathcal{C}_{G}(V)_{\leq w}$ that $\mathcal{O}_{V} \in \mathcal{C}_{G}(V)_{\leq 0}$, so its subsheaf $\mathcal{I}$ is in $\mathcal{C}_{G}(V)_{\leq 0}$ as well. (We cannot yet invoke Proposition 3.12 on $V$, however.) Therefore, $\left.t_{*} \mathcal{F}\right|_{V} \otimes \mathcal{I} \in \mathcal{C}_{G}(V)_{\leq w}$, so its quotient $\mathcal{I} \mathcal{H}$ must be in $\mathcal{C}_{G}(V)_{\leq w}$ as well. Since we also have $\mathcal{I H} \in \mathcal{C}_{G}(V)_{\geq w+1}$, we conclude that $\mathcal{I H}=0$, and hence that $\mathcal{H}$ is actually supported on $V_{1}$ : we have $\mathcal{H} \simeq t_{*} \mathcal{H}_{1}$ for 
some $\mathcal{H}_{1} \in \mathcal{C}_{G}\left(V_{1}\right)$. The sequence $\left.\left.\left.0 \rightarrow \mathcal{G}\right|_{V_{1}} \rightarrow \mathcal{H}_{1}\right|_{V_{1}} \rightarrow \mathcal{F}\right|_{V_{1}} \rightarrow 0$ splits because $\operatorname{Ext}^{1}\left(\left.\mathcal{F}\right|_{V_{1}},\left.\mathcal{G}\right|_{V_{1}}\right)=0$, and hence so does $\left.\left.0 \rightarrow t_{*} \mathcal{G}\right|_{V} \rightarrow \mathcal{H} \rightarrow t_{*} \mathcal{F}\right|_{V} \rightarrow 0$.

Proposition 4.5. Assume that $Z$ is endowed with a weak s-structure. Then the induced pre-s-structure on $Z^{\prime}$ is also a weak s-structure.

Proof. Suppose $\mathcal{G} \in \tilde{\mathcal{C}}_{G}\left(Z^{\prime}\right)_{\geq w}$. We will simultaneously prove axiom (S7) and the condition in Definition 4.3 by induction on the invariant $\ell(\mathcal{G})$ that was introduced in the proof of Proposition 4.1 .

If $\ell(\mathcal{G})=1$, then we have $\mathcal{G} \simeq t_{*} \mathcal{G}_{1}$ for some $\mathcal{G}_{1} \in \tilde{\mathcal{C}}_{G}(Z)_{\geq w}$, so every quotient of $\mathcal{G}$ is in $\tilde{\mathcal{C}}_{G}\left(Z^{\prime}\right)_{\geq w}$ because every quotient of $\mathcal{G}_{1}$ is in $\tilde{\mathcal{C}}_{G}(Z)_{\geq w}$. Next, observe that for any $\mathcal{F} \in \mathcal{C}_{G}\left(Z^{\prime}\right)_{\leq w-1}$, we have a sequence

$$
\operatorname{Ext}^{1}\left(\mathcal{F} / \mathcal{I} \mathcal{F}, t_{*} \mathcal{G}_{1}\right) \rightarrow \operatorname{Ext}^{1}\left(\mathcal{F}, t_{*} \mathcal{G}_{1}\right) \rightarrow \operatorname{Ext}^{1}\left(\mathcal{I} \mathcal{F}, t_{*} \mathcal{G}_{1}\right)
$$

that is exact at the middle term. Recall that $\mathcal{C}_{G}\left(Z^{\prime}\right)_{\leq w-1}$ is a Serre subcategory of $\mathcal{C}_{G}\left(Z^{\prime}\right)$. Thus, an argument by induction on $\ell(\mathcal{F})$, combined with Lemma 4.4. shows that there exists an open subscheme $V \subset Z^{\prime}$ with $\operatorname{Ext}^{1}\left(\left.\mathcal{F}\right|_{V},\left.t_{*} \mathcal{G}_{1}\right|_{V}\right)=0$.

Now, suppose $\ell(\mathcal{G})>1$. We claim that $\mathcal{G} / \mathcal{I} \mathcal{G} \in \tilde{\mathcal{C}}_{G}\left(Z^{\prime}\right)_{\geq w}$. Suppose instead that $\mathcal{G} / \mathcal{I} \mathcal{G} \notin \tilde{\mathcal{C}}_{G}\left(Z^{\prime}\right)_{\geq w}$, and let $\mathcal{H}=\sigma_{\leq w-1}(\mathcal{G} / \mathcal{I} \mathcal{G})$. The natural map $\mathcal{H} \rightarrow \mathcal{G} / \mathcal{I} \mathcal{G}$ remains nonzero upon restriction to any dense open subset of $Z^{\prime}$. Since $\ell(\mathcal{I} \mathcal{G})<$ $\ell(\mathcal{G})$, we know inductively that there is an open subscheme $V_{1} \subset Z^{\prime}$ such that $\operatorname{Ext}^{1}\left(\left.\mathcal{H}\right|_{V_{1}},\left.\mathcal{I} \mathcal{G}\right|_{V_{1}}\right)=0$. By replacing $V_{1}$ with a smaller open subscheme if necessary, we may also assume that $\operatorname{Hom}\left(\left.\mathcal{H}\right|_{V_{1}},\left.\mathcal{G}\right|_{V_{1}}\right)=0$. Consider the exact sequence

$$
\operatorname{Hom}\left(\left.\mathcal{H}\right|_{V_{1}},\left.\mathcal{G}\right|_{V_{1}}\right) \rightarrow \operatorname{Hom}\left(\left.\mathcal{H}\right|_{V_{1}},\left.(\mathcal{G} / \mathcal{I} \mathcal{G})\right|_{V_{1}}\right) \rightarrow \operatorname{Ext}^{1}\left(\left.\mathcal{H}\right|_{V_{1}},\left.\mathcal{I} \mathcal{G}\right|_{V_{1}}\right)
$$

We now have a contradiction: the first and last terms vanish, but the middle term does not. Thus, it must be that $\mathcal{G} / \mathcal{I} \mathcal{G} \in \tilde{\mathcal{C}}_{G}\left(Z^{\prime}\right)_{\geq w}$. Now, any quotient of $\mathcal{G}$ is an extension of a quotient of $\mathcal{G} / \mathcal{I} \mathcal{G}$ be a quotient of $\mathcal{I} \mathcal{G}$. The latter two are now known to be in $\tilde{\mathcal{C}}_{G}\left(Z^{\prime}\right)_{\geq w}$, so we see that any quotient of $\mathcal{G}$ is in $\tilde{\mathcal{C}}_{G}\left(Z^{\prime}\right)_{\geq w}$.

Next, let $\mathcal{F} \in \mathcal{C}_{G}\left(Z^{\prime}\right)_{\leq w-1}$. By invoking the inductive assumption twice, we may find a dense open subscheme $V \Subset Z^{\prime}$ such that the first and last terms of the following exact sequence both vanish:

$$
\operatorname{Ext}^{1}\left(\left.\mathcal{F}\right|_{V},\left.\mathcal{I} \mathcal{G}\right|_{V}\right) \rightarrow \operatorname{Ext}^{1}\left(\left.\mathcal{F}\right|_{V},\left.\mathcal{G}\right|_{V}\right) \rightarrow \operatorname{Ext}^{1}\left(\left.\mathcal{F}\right|_{V},\left.(\mathcal{G} / \mathcal{I} \mathcal{G})\right|_{V}\right) .
$$

We then see that $\operatorname{Ext}^{1}\left(\left.\mathcal{F}\right|_{V},\left.\mathcal{G}\right|_{V}\right)=0$ as well, as desired.

We now turn to axiom ( 88 . Because $\tilde{\mathcal{C}}_{G}\left(Z^{\prime}\right)_{\geq w}$ is now known to be a Serre subcategory, this axiom can be deduced by induction on $\ell(\mathcal{F})$ from the corresponding statement on $Z$, using the same argument that was given for axiom ( $\mathrm{S} 6$ ) in the proof of Proposition 4.1 .

\section{The Gluing Theorem for $s$-Structures}

Given an $s$-structure on a closed subscheme of $X$, and another $s$-structure on its open complement, we show in this section how to produce an $s$-structure on $X$. The latter is said to be obtained by gluing the two given $s$-structures. Gluing cannot be carried out for arbitrary $s$-structures, however; certain compatibility conditions must hold.

In particular, consider the following condition on a closed subscheme:

Definition 5.1. Let $Z \subset X$ be a closed subscheme with a pre-s-structure. $Z$ is said to be adhesive if it satisfies the following two conditions: 
(A1) The ideal sheaf $\mathcal{I} \subset \mathcal{O}_{X}$ corresponding to $Z$ is in $\mathcal{C}_{G}^{\text {loc }}(X, Z)_{\leq 0}$.

(A2) For any closed subscheme $Y \subset Z$, any $\mathcal{F} \in \mathcal{C}_{G}^{\text {loc }}(X, Y)_{\leq w}$, and any $r \geq 0$, there is a dense open subscheme $V \Subset_{Y} X$ such that $\operatorname{Ext}^{r}\left(\left.\mathcal{F}\right|_{V},\left.\mathcal{G}\right|_{V}\right)=0$ for all $\mathcal{G} \in \mathcal{C}_{G}^{\text {supp }}(X, Y)_{\geq w+1}$.

Note that axiom (A1) is the same as condition (4.1), so the results of Section 4 can be applied to adhesive subschemes. Here, $X$ is not assumed to have an $s$ structure, but if $X$ does happen to have one, it is easy to see that every closed subscheme (with the induced $s$-structure) is automatically adhesive. Indeed, it follows from Proposition 3.12 that $\mathcal{I} \in \mathcal{C}_{G}(X)_{\leq 0}$, and hence, from the description of the $s$-structure on $Z$ in Proposition 3.6, that $\mathcal{I} \in \mathcal{C}_{G}^{\text {loc }}(X, Z)_{\leq 0}$. Axiom (A2) is merely a special case of axiom (\$9) for the $s$-structure on $X$. Thus, adhesiveness of the closed subscheme must be a necessary condition for a gluing theorem to hold.

We will begin with a preliminary version, in which we only produce a pre-sstructure.

Proposition 5.2. Let $i: Z \hookrightarrow X$ be a closed subscheme, and let $j: U \rightarrow X$ be the complementary open subscheme. Suppose that $Z$ is endowed with an s-structure, and that $U$ is endowed with a pre-s-structure. Define two full subcategories of $\mathcal{C}_{G}(X)$ by

$$
\begin{aligned}
& \mathcal{C}_{G}(X)_{\leq w}=\left\{\mathcal{F} \in \mathcal{C}_{G}(X) \mid j^{*} \mathcal{F} \in \mathcal{C}_{G}(U)_{\leq w} \text { and } \mathcal{F} \in \mathcal{C}_{G}^{\text {loc }}(X, Z)_{\leq w}\right\}, \\
& \mathcal{C}_{G}(X)_{\geq w}=\left\{\mathcal{G} \in \mathcal{C}_{G}(X) \mid j^{*} \mathcal{G} \in \mathcal{C}_{G}(U)_{\geq w} \text { and } \Gamma_{Z} \mathcal{G} \in \mathcal{C}_{G}^{\text {supp }}(X, Z)_{\geq w}\right\} .
\end{aligned}
$$

Assume that $Z$ is adhesive, and that the following condition holds:

$$
\text { For every } \mathcal{F}^{\prime} \in \mathcal{C}_{G}(U)_{\leq w} \text {, there exists some } \mathcal{F} \in \mathcal{C}_{G}(X)_{\leq w} \text { with } j^{*} \mathcal{F} \simeq \mathcal{F}^{\prime} .
$$

Then, the above categories define the unique pre-s-structure on $X$ whose restrictions to $U$ and $Z$ coincide with the given ones.

Proof. We proceed by noetherian induction: we assume that for any closed subscheme of $X$, the theorem is already known. The base cases are those in which either $Z$ or $U$ is empty. The theorem is true in both these cases: the former is trivial, and the latter reduces to Proposition 4.1. Henceforth, assume that $U$ and $Z$ are both nonempty.

It is easy to see that $\mathcal{C}_{G}(X)_{\leq w}$ is a Serre subcategory of $\mathcal{C}_{G}(X)$, using Proposition 4.2. the exactness of $j^{*}$, and the fact that $\mathcal{C}_{G}(U)_{\leq w}$ is a Serre subcategory of $\mathcal{C}_{G}(U)$. Similarly, $\mathcal{C}_{G}(X)_{\geq w}$ is closed under subobjects and extensions, because $\mathcal{C}_{G}(U)_{\geq w}$ and $\mathcal{C}_{G}^{\text {supp }}(X, Z)_{\geq w}$ are, and $j^{*}$ and $\Gamma_{Z}$ are left exact.

Next, we consider axiom (\$3). Suppose $\mathcal{F} \in \mathcal{C}_{G}(X)_{\leq w}$ and $\mathcal{G} \in \mathcal{C}_{G}(X)_{\geq w+1}$, and let $V \subset X$ be an open subscheme. There is some closed subscheme structure $i_{Z^{\prime}}: Z^{\prime} \hookrightarrow X$ on the underlying space of $Z$ and some sheaf $\mathcal{G}_{1} \in \mathcal{C}_{G}\left(Z^{\prime}\right)_{\geq w+1}$ such that $\Gamma_{Z} \mathcal{G} \simeq i_{Z^{\prime} *} \mathcal{G}_{1}$. Let $t: Z \hookrightarrow Z^{\prime}$ be the inclusion map. Since $i^{*} \mathcal{F} \simeq t^{*} i_{Z^{\prime}}^{*} \mathcal{F}$ is assumed to be in $\mathcal{C}_{G}(Z)_{\leq w}$, we see that $i_{Z^{\prime}}^{*} \mathcal{F} \in \mathcal{C}_{G}\left(Z^{\prime}\right)_{\leq w}$, and then we have

$$
\operatorname{Hom}\left(\left.\mathcal{F}\right|_{V},\left.\Gamma_{Z} \mathcal{G}\right|_{V}\right) \simeq \operatorname{Hom}\left(\left.\mathcal{F}\right|_{V},\left.i_{Z^{\prime} *} \mathcal{G}_{1}\right|_{V}\right) \simeq \operatorname{Hom}\left(\left.i_{Z^{\prime}}^{*} \mathcal{F}\right|_{V \cap Z^{\prime}},\left.\mathcal{G}_{1}\right|_{V \cap Z^{\prime}}\right)=0 .
$$

In addition, we clearly have $\operatorname{Hom}\left(j^{*} \mathcal{F}, j^{*} \mathcal{G}\right)=0$, so from the exact sequence

$$
0 \rightarrow \operatorname{Hom}\left(\left.\mathcal{F}\right|_{V},\left.\Gamma_{Z} \mathcal{G}\right|_{V}\right) \rightarrow \operatorname{Hom}\left(\left.\mathcal{F}\right|_{V},\left.\mathcal{G}\right|_{V}\right) \rightarrow \operatorname{Hom}\left(\left.j^{*} \mathcal{F}\right|_{V \cap U},\left.j^{*} \mathcal{G}\right|_{V \cap U}\right),
$$

we see that $\operatorname{Hom}\left(\left.\mathcal{F}\right|_{V},\left.\mathcal{G}\right|_{V}\right)=0$, and hence $\operatorname{Hom}(\mathcal{F}, \mathcal{G})=0$.

Next, suppose $\mathcal{G} \in \mathcal{C}_{G}(X)_{\leq w}^{\perp}$. We will show that if $U^{\prime} \subset X$ is any open subscheme containing $U$, then we have $\operatorname{Hom}\left(\left.\mathcal{F}\right|_{U^{\prime}},\left.\mathcal{G}\right|_{U^{\prime}}\right)=0$ for all $\mathcal{F} \in \mathcal{C}_{G}(X)_{\leq w}$. (This fact 
will be used to establish axiom (S4).) We proceed by noetherian induction on the complement of $U^{\prime}$. In the case $U^{\prime}=X$, there is nothing to prove; $\operatorname{Hom}(\mathcal{F}, \mathcal{G})=0$ by assumption. Now, suppose $U^{\prime} \neq X$. Let $Y$ be any closed subscheme structure on the complement $X \backslash U^{\prime}$ that also makes it into a closed subscheme of $Z$. Then, let $i_{Y^{\prime}}: Y^{\prime} \hookrightarrow X$ be a closed subscheme structure on the underlying space of $Y$ such that $\Gamma_{Y} \mathcal{G}$ is supported on $Y^{\prime}$ : we have $\Gamma_{Y} \mathcal{G} \simeq i_{Y^{\prime} *} \mathcal{G}_{1}$ for some $\mathcal{G}_{1} \in \mathcal{C}_{G}\left(Y^{\prime}\right)$. Now, from the injective map

$$
\operatorname{Hom}\left(\mathcal{F}, \Gamma_{Y} \mathcal{G}\right) \rightarrow \operatorname{Hom}(\mathcal{F}, \mathcal{G})
$$

we see that $\operatorname{Hom}\left(\mathcal{F}, i_{Y^{\prime} *} \mathcal{G}_{1}\right)=0$ for all $\mathcal{F} \in \mathcal{C}_{G}(X)_{\leq w}$. In particular, considering just those $\mathcal{F}$ of the form $\mathcal{F} \simeq i_{Y^{\prime} *} \mathcal{F}_{1}$ with $\mathcal{F}_{1} \in \mathcal{C}_{G}\left(Y^{\prime}\right)_{\leq w}$, we see that $\mathcal{G}_{1} \in$ $\mathcal{C}_{G}\left(Y^{\prime}\right)_{\geq w+1}$, and hence $\Gamma_{Y} \mathcal{G} \in \mathcal{C}_{G}^{\text {supp }}(X, Y)_{\geq w+1}$.

Therefore, because $Z$ is adhesive, there is an open subscheme $U^{\prime \prime} \Subset_{Y} X$ such that $\operatorname{Ext}^{1}\left(\left.\mathcal{F}\right|_{U^{\prime \prime}},\left.\Gamma_{Y} \mathcal{G}\right|_{U^{\prime \prime}}\right)=0$. Form the long exact sequence

$$
\begin{aligned}
0 \rightarrow \operatorname{Hom}\left(\left.\mathcal{F}\right|_{U^{\prime \prime}},\left.\Gamma_{Y} \mathcal{G}\right|_{U^{\prime \prime}}\right) \rightarrow \operatorname{Hom}\left(\left.\mathcal{F}\right|_{U^{\prime \prime}},\left.\mathcal{G}\right|_{U^{\prime \prime}}\right) & \rightarrow \\
\operatorname{Hom}\left(\left.\mathcal{F}\right|_{U^{\prime \prime}}, \mathcal{G} /\left.\Gamma_{Y} \mathcal{G}\right|_{U^{\prime \prime}}\right) & \rightarrow \operatorname{Ext}^{1}\left(\left.\mathcal{F}\right|_{U^{\prime \prime}},\left.\Gamma_{Z} \mathcal{G}\right|_{U^{\prime \prime}}\right) \rightarrow \cdots .
\end{aligned}
$$

Recall our inductive assumption: $U^{\prime \prime}$ is strictly larger than $U^{\prime}$ (since $U^{\prime \prime} \cap Y$ is dense in $Y$ ), so we assume that $\operatorname{Hom}\left(\left.\mathcal{F}\right|_{U^{\prime \prime}},\left.\mathcal{G}\right|_{U^{\prime \prime}}\right)$ is known to be 0 for all $\mathcal{F} \in \mathcal{C}_{G}(X)_{\leq w}$. It follows that $\operatorname{Hom}\left(\left.\mathcal{F}\right|_{U^{\prime \prime}}, \mathcal{G} /\left.\Gamma_{Y} \mathcal{G}\right|_{U^{\prime \prime}}\right)=0$. The same holds if we replace $\mathcal{F}$ by any subsheaf (since $\mathcal{C}_{G}(X)_{\leq w}$ is closed under subobjects), so by Lemma 2.5 we have that $\operatorname{Hom}\left(\left.\mathcal{F}\right|_{U^{\prime}},\left.\mathcal{G}\right|_{U^{\prime}}\right)=0$, as desired.

Thus, $\operatorname{Hom}\left(\left.\mathcal{F}\right|_{U^{\prime}},\left.\mathcal{G}\right|_{U^{\prime}}\right)=0$ for any open subscheme containing $U$ and any $\mathcal{F} \in$ $\mathcal{C}_{G}(X)_{\leq w}$. In particular, this holds for $U^{\prime}=U$. By assumption, every sheaf in $\mathcal{C}_{G}(U)_{\leq w}$ occurs as the restriction to $U$ of some $\mathcal{F} \in \mathcal{C}_{G}(X)_{\leq w}$, so the fact that $\operatorname{Hom}\left(j^{*} \mathcal{F}, j^{*} \mathcal{G}\right)=0$ for all $\mathcal{F} \in \mathcal{C}_{G}(X)_{\leq w}$ implies that $j^{*} \mathcal{G} \in \mathcal{C}_{G}(U)_{\geq w+1}$. On the other hand, we saw in the preceding paragraph that $\Gamma_{Y} \mathcal{G} \in \mathcal{C}_{G}^{\text {supp }}(X, Y)_{\geq w+1}$ for any closed subscheme $Y \subset Z$. This holds in particular for $Y=Z$. We are able to conclude, at long last, that $\mathcal{G} \in \mathcal{C}_{G}(X)_{<w}^{\perp}$ implies that $\mathcal{G} \in \mathcal{C}_{G}(X)_{\geq w+1}$.

Since axiom ( $(3)$ is already established, we see that $\mathcal{C}_{G}(X)_{\leq w}^{\perp}=\mathcal{C}_{G}(X)_{\geq w+1}$, and then by Proposition 2.7, axiom (S4) holds.

Given $\mathcal{F} \in \mathcal{C}_{G}(X)$, there is some $w_{1} \in \mathbb{Z}$ such that $j^{*} \mathcal{F} \in \mathcal{C}_{G}(U)_{\geq w_{1}}$. On the other hand, suppose $\Gamma_{Z} \mathcal{F} \simeq i_{Z^{\prime} *} \mathcal{F}_{1}$, where $i_{Z^{\prime}}: Z^{\prime} \hookrightarrow X$ is a closed subscheme structure on $\underline{Z}$. There is some $w_{2}$ such that $\mathcal{F}_{1} \in \mathcal{C}_{G}\left(Z^{\prime}\right)_{\geq w_{2}}$. We then clearly have $\mathcal{F} \in \mathcal{C}_{G}(X)_{\geq \min \left\{w_{1}, w_{2}\right\}}$. Similarly, we know that $j^{*} \mathcal{F} \in \mathcal{C}_{G}(U)_{\leq v_{1}}$ and $i^{*} \mathcal{F} \in$ $\mathcal{C}_{G}(Z)_{\leq v_{2}}$ for some $v_{1}, v_{2} \in \mathbb{Z}$, so $\mathcal{F} \in \mathcal{C}_{G}(X)_{\leq \max \left\{v_{1}, v_{2}\right\}}$. Thus, axiom (\$5 holds.

Axiom ( $(\sqrt{6})$ is immediate from the fact that $j^{*}(\mathcal{F} \otimes \mathcal{G}) \simeq j^{*} \mathcal{F} \otimes j^{*} \mathcal{G}$ and $i^{*}(\mathcal{F} \otimes$ $\mathcal{G}) \simeq i^{*} \mathcal{F} \otimes i^{*} \mathcal{G}$.

To show uniqueness, suppose there were another pre-s-structure $\left(\left\{\mathcal{C}_{\leq w}^{\prime}\right\},\left\{\mathcal{C}_{\geq w}^{\prime}\right\}\right)$ on $X$ whose restrictions to $U$ and $Z$ were the given $s$-structures on those schemes. Since $j^{*}$ and $i^{*}$ must both be right $s$-exact, we see that $\mathcal{F} \in \mathcal{C}_{\leq w}^{\prime}$ implies that $j^{*} \mathcal{F} \in \mathcal{C}_{G}(U)_{\leq w}$ and $i^{*} \mathcal{F} \in \mathcal{C}_{G}(Z)_{\leq w}$. In other words, $\mathcal{C}_{\leq w}^{\prime} \subset \mathcal{C}_{G}(X)_{\leq w}$. On the

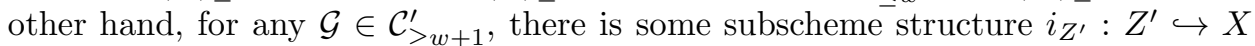
and some sheaf $\mathcal{G}_{1} \in \mathcal{C}_{G}\left(Z^{\prime}\right)$ such that $\Gamma_{Z} \mathcal{G} \simeq i_{Z^{\prime} *} i_{Z^{\prime}} \mathcal{G}$. Since $i_{Z^{\prime}}^{!}$is left $s$-exact, we see that $\Gamma_{Z} \mathcal{G} \in \mathcal{C}_{G}^{\text {supp }}(X, Z)_{\geq w+1}$. The functor $j^{*}$ is also left $s$-exact, so in fact we must have $\mathcal{G} \in \mathcal{C}_{G}(X)_{\geq w+1}$. We deduce that $\mathcal{C}_{\geq w}^{\prime} \subset \mathcal{C}_{G}(X)_{\leq w}$. Now, the equalities $\mathcal{C}_{\leq w}^{\prime \perp}=\mathcal{C}_{\geq w+1}^{\prime}$ and $\mathcal{C}_{G}(X)_{\leq w}^{\perp}=\mathcal{C}_{G}(X)_{\geq w+1}$ together imply that $\mathcal{C}_{\leq w}^{\prime}=\mathcal{C}_{G}(X)_{\leq w}$ and $\mathcal{C}_{\geq w}^{\prime}=\mathcal{C}_{G}(X)_{\geq w}$. 
The main result of this section is the following.

Theorem 5.3. Let $i: Z \hookrightarrow X$ be a closed subscheme of $X$, and let $j: U \hookrightarrow X$ be the complementary open subscheme. Assume that $U$ and $Z$ are both endowed with s-structures, that $Z$ is adhesive, and that condtion (5.2) holds. Then the pres-structure on $X$ obtained by gluing is an s-structure.

Proof. Let $\kappa: Y \hookrightarrow X$ be a closed subscheme. Let $U_{1}=Y \cap U$, and let $V_{1} \subset Y$ be the open subscheme complementary to $Y \backslash \bar{U}_{1}$. Clearly, $U_{1} \cup V_{1}$ is a dense open subscheme of $Y$. Now, $U_{1}$ carries an $s$-structure because it is a closed subscheme of $U$. On the other hand, $V_{1}$ is a possibly nonreduced scheme whose subscheme $V_{1} \cap Z$ has the same underlying topological space. $V_{1} \cap Z$ carries an $s$-structure induced from $Z$, so by Proposition 4.5. $V_{1}$ has at least a weak $s$-structure. Therefore, $U_{1} \cup V_{1}$ carries a weak $s$-structure.

Let $h: U_{1} \cup V_{1} \hookrightarrow Y$ be the inclusion map. We know that $h^{*}$ is exact, and by Lemma 3.8, we know that $\mathcal{F} \in \tilde{\mathcal{C}}_{G}(Y)_{\geq w}$ if and only if $h^{*} \mathcal{F} \in \tilde{\mathcal{C}}_{G}\left(U_{1} \cup V_{1}\right)_{\geq w}$. These observations, combined with the formula $h^{*}(\mathcal{F} \otimes \mathcal{G}) \simeq h^{*} \mathcal{F} \otimes h^{*} \mathcal{G}$, imply that axioms (S7) and (S8) for $Y$ follow from the fact that $U_{1} \cup V_{1}$ has an almost $s$-structure.

Finally, we consider axiom (S9). Suppose $\mathcal{F} \in \mathcal{C}_{G}(X)_{\leq w}$, and fix an integer $r \geq 0$. Since $U$ has an $s$-structure, we know that there is an open subscheme $V^{\prime} \Subset_{U_{1}} U$ such that $\operatorname{Ext}^{r}\left(\left.\mathcal{F}\right|_{V^{\prime}},\left.\kappa_{*} \mathcal{G}\right|_{V^{\prime}}\right)=0$ for all $\mathcal{G} \in \tilde{\mathcal{C}}_{G}(Y) \geq w+1$. Next, we would like to find an open subscheme $V^{\prime \prime} \Subset_{V_{1}} X \backslash \bar{U}_{1}$ such that $\operatorname{Ext}^{r}\left(\left.\mathcal{F}\right|_{V^{\prime \prime}},\left.\kappa_{*} \mathcal{G}\right|_{V^{\prime \prime}}\right)=0$. If we had such a $V^{\prime \prime}$, note that it would be open as a subscheme of $X$ and disjoint from $V^{\prime}$. Their union $V=V^{\prime} \cup V^{\prime \prime}$ (which would satisfy $V \Subset_{Y} X$ ) would then be an open subscheme of the sort required by axiom (S9) for $Y$.

Let $V_{2}=V_{1} \cap Z$. Note that $V_{2}$ is a closed subscheme of $V_{1}$ with the same underlying topological space as $V_{1} . V_{2}$ is also a locally closed subscheme of $Z$. We have $\left.\kappa_{*} \mathcal{G}\right|_{X \backslash \bar{U}_{1}} \in \mathcal{C}_{G}^{\text {supp }}\left(X \backslash \bar{U}_{1}, V_{2}\right)_{\geq w+1}$, so the desired open subscheme $V^{\prime \prime}$ comes from condition $(\mathrm{A} 2)$ of Definition 5.1 applied to the closed subscheme $\bar{V}_{2} \subset Z$.

\section{Duality}

This section is devoted to studying the interaction between $s$-structures and Grothendieck-Serre duality. The results established here will be essential to the construction of the staggered $t$-structure in Sections 7 and 8 .

Throughout, we assume that $X$ is endowed with an $s$-structure. Fix an equivariant dualizing complex $\omega_{X}$ on $X$, and recall that we denote the Grothendieck-Serre duality functor by $\mathbb{D}=R \mathcal{H} \operatorname{om}\left(\cdot, \omega_{X}\right)$.

For any point $x \in X$, let $k(x)$ be the residue field of the local ring $\mathcal{O}_{x}$, and let $I_{x}$ be the injective hull of $k(x)$ in the category $\mathcal{O}_{x}$-mod of $\mathcal{O}_{x}$-modules. Recall that for any complex of coherent sheaves $\mathcal{F}$ on $X$, the local cohomology groups $H_{x}^{i}(\mathcal{F})=$ $H^{i}\left(R \Gamma_{x}(\mathcal{F})\right)$ are artinian $\mathcal{O}_{x}$-modules. Here, and throughout the remainder of the paper, points (in contrast to subschemes) are not to be thought of in any equivariant sense. Accordingly, $\mathcal{O}_{x}$-modules are modules without any group action, and $R \Gamma_{x}$ is to be computed after passing to the nonequivariant derived category.

Lemma 6.1. At any point $x \in X$, the complex $R \Gamma_{x}\left(\omega_{X}\right)$ is concentrated in one degree $d$. In addition, if $x$ is a generic point of $X$, there is an open subscheme $U$ containing $x$ such that $\left.\omega_{X}\right|_{U}$ is concentrated in degree $d$. 
Proof. The fact that $R \Gamma_{x}\left(\omega_{X}\right)$ is concentrated in a single degree follows from $\underline{\mathrm{H}}$, Proposition V.3.4]. When $x$ is generic, the existence of the desired open subscheme $U$ is given by [B1, Lemma 2].

For the remainder of the section, we will usually assume that $\omega_{X}$ is a sheaf, i.e., a complex concentrated in degree 0. According to the previous lemma, this situation can always be achieved by replacing $X$ by a suitable open subscheme, and by shifting $\omega_{X}$.

Lemma 6.2. Assume $\omega_{X}$ is a sheaf. There is an open subscheme $U \subset X$ and an integer $e$ such that for all open subschemes $V \subset U,\left.\omega_{X}\right|_{V} \in \mathcal{C}_{G}(V)_{\geq e}$ and $\left.\omega_{X}\right|_{V} \notin \mathcal{C}_{G}(V)_{\geq e+1}$.

Proof. By axiom (S5), there is a $v$ such that $\omega_{X} \in \mathcal{C}_{G}(X)_{\leq v}$. Choose a generic point $x \in X$. Consider the sequence of subobjects

$$
\cdots \subset \sigma_{\leq v-1} \omega_{X} \subset \sigma_{\leq v} \omega_{X}=\omega_{X} .
$$

Applying the left exact functor $\Gamma_{x}$ gives us a decreasing sequence of subobjects of $\Gamma_{x} \omega_{X} \simeq I_{x}$, and the latter is an artinian $\mathcal{O}_{x}$-module. Thus, there is some integer $m$ such that $\Gamma_{x} \sigma_{\leq w} \omega_{X}=\Gamma_{x} \sigma_{\leq m} \omega_{X}$ for all $w \leq m$. Indeed, it follows then that

$$
\Gamma_{x}\left(\bigcap_{w \leq m} \sigma_{\leq w} \omega_{X}\right)=\Gamma_{x} \sigma_{\leq m} \omega_{X},
$$

so by axiom ( $\mathrm{S} 5$ ), both sides of this equation must be 0 . Now, assume that $m$ is in fact the largest integer such that $\Gamma_{x} \sigma_{\leq m} \omega_{X}=0$. Since $\Gamma_{x} \sigma_{\leq m} \omega_{X}=0$, there is an irreducible open subscheme $U \subset X$ containing $x$ such that $\left.\left(\sigma_{\leq m} \omega_{X}\right)\right|_{U}=0$, and hence $\left.\left(\sigma_{<w} \omega_{X}\right)\right|_{U}=0$ for all $w \leq m$. On the other hand, the fact that $\Gamma_{x} \sigma_{\leq m+1} \omega_{X} \neq 0$ implies that for every open subscheme $V$ containing $x$ (and in particular every $V \subset U)$, we have $\left.\left(\sigma_{\leq m+1} \omega_{X}\right)\right|_{V} \neq 0$. Thus, the open subscheme $U$ and the integer $e=m+1$ have the desired properties.

Combining the above two lemmas leads to the following definition.

Definition 6.3. Assume $X$ is irreducible. The altitude of $X$, denoted alt $X$, is the unique integer such that for any open subscheme $U \subset X$, there is an open subscheme $V \subset U$ such that $\left.\omega_{X}\right|_{V}$ is concentrated in a single degree $d$, and $\left.\omega_{X}[d]\right|_{V} \in \mathcal{C}_{G}(V)_{\geq \text {alt } X}$ but $\left.\omega_{X}\right|_{V} \notin \mathcal{C}_{G}(V)_{\geq \text {alt } X+1}$.

Below, we will see that the altitude of a closed subscheme does not depend on the choice of closed subscheme structure. This result depends on the following useful lemma.

Lemma 6.4. Assume that $\omega_{X}$ is a sheaf. If $\omega_{X}$ has a subsheaf $\mathcal{F}$ that is a direct sum of two smaller subsheaves, $\mathcal{F} \simeq \mathcal{F}_{1} \oplus \mathcal{F}_{2}$, then there is an open subscheme $U \subset X$ such that either $\left.\mathcal{F}_{1}\right|_{U}=0$ or $\left.\mathcal{F}_{2}\right|_{U}=0$.

Proof. Let $x$ be a generic point of $X$. Applying $\Gamma_{x}$, we get an injective map

$$
\Gamma_{x} \mathcal{F}_{1} \oplus \Gamma_{x} \mathcal{F}_{2} \rightarrow \Gamma_{x} \omega_{X} \simeq I_{x} .
$$

But every submodule of $I_{x}$ contains the residue field $k(x)$, so no submodule of $\Gamma_{x} \omega_{X}$ is a direct sum of two smaller nonzero submodules. Thus, either $\Gamma_{x} \mathcal{F}_{1}$ or $\Gamma_{x} \mathcal{F}_{2}$ must be 0 , and therefore there is an open subscheme $U \subset Y$ on which either $\mathcal{F}_{1}$ or $\mathcal{F}_{2}$ vanishes. 
Proposition 6.5. Let $X^{\prime}$ be a nonreduced irreducible scheme, and let $t: X \hookrightarrow X^{\prime}$ be a closed subscheme with the same underlying space. Then $\operatorname{alt} X=\operatorname{alt} X^{\prime}$.

Proof. By replacing $X^{\prime}$ by a suitable open subscheme and shifting if necessary, we may assume that $\omega_{X^{\prime}}$ and $\omega_{X}$ are both sheaves, and that $\omega_{X^{\prime}} \in \mathcal{C}_{G}\left(X^{\prime}\right) \geq$ alt $X^{\prime}$ and $\omega_{X} \in \mathcal{C}_{G}(X)_{\geq \text {alt } X}$. Let $\mathcal{I} \subset \mathcal{O}_{X^{\prime}}$ be the nilpotent ideal sheaf corresponding to $X \subset X^{\prime}$. Then $\mathcal{O}_{X^{\prime}} / \mathcal{I} \simeq \mathcal{O}_{X}$, and $\omega_{X} \simeq \mathcal{H}$ om $\left(\mathcal{O}_{X^{\prime}} / \mathcal{I}, \omega_{X^{\prime}}\right)$. Thus, $\omega_{X}$ can naturally be identified with a subsheaf of $\omega_{X^{\prime}}$. It follows that alt $X \geq \operatorname{alt} X^{\prime}$.

Now, suppose that alt $X>$ alt $X^{\prime}$. Then $\omega_{X}$, regarded as a subsheaf of $\omega_{X^{\prime}}$, has trivial intersection with the nonzero sheaf $\sigma_{\leq \text {alt } X^{\prime}} \omega_{X^{\prime}}$. That is, $\omega_{X^{\prime}}$ contains the direct sum

$$
\sigma_{\leq} \text {alt } X^{\prime} \omega_{X^{\prime}} \oplus \omega_{X}
$$

as a subsheaf. According to Lemma 6.4, we can replace $X^{\prime}$ by an open subscheme and then assume that one of $\sigma_{\leq \text {alt } X^{\prime}} \omega_{X^{\prime}}$ or $\omega_{X}$ vanishes. But this is clearly absurd. We conclude that alt $X=\operatorname{alt} \bar{X}^{\prime}$.

The last few results in this section will play an essential role in the developments of Sections 7 and 8 .

Lemma 6.6. Assume that $X$ is irreducible and that $\omega_{X}$ is a sheaf. For any $\mathcal{F} \in$ $\mathcal{C}_{G}(X)$, there is an open subscheme $U \subset X$ such that $\left.\mathbb{D}(\mathcal{F})\right|_{U}$ is a sheaf.

Proof. Let $x$ be a generic point of $X$, and let $\mathcal{G}=\mathbb{D}(\mathcal{F})$. Then we have $R \Gamma_{x} \mathcal{G} \simeq$ $R \operatorname{Hom}\left(\mathcal{F}_{x}, I_{x}\right)$, but since $I_{x}$ is injective, this is $\operatorname{simply} \operatorname{Hom}\left(\mathcal{F}_{x}, I_{x}\right)$. Since $R \Gamma_{x} \mathcal{G}$ is concentrated in degree 0 , there is an open subscheme $U \subset X$ such that $\left.\mathcal{G}\right|_{U}$ is a sheaf.

Lemma 6.7. Assume that $X$ is irreducible and that $\omega_{X}$ is a sheaf. Let $\mathcal{F} \in \mathcal{C}_{G}(X)$ be pure of step $w$. Then there is an open subscheme $U \subset X$ such that $\left.\mathbb{D}(\mathcal{F})\right|_{U}$ is a sheaf, and such that it is pure of step alt $X-w$.

(Recall that $\mathcal{F}$ is pure of step $w$ if it lies in $\mathcal{C}_{G}(X)_{\leq w} \cap \mathcal{C}_{G}(X)_{\geq w}$.)

Proof. Let $x$ be a generic point of $X$, and let $\mathcal{G}=\mathbb{D}(\mathcal{F})$. Then we have $R \Gamma_{x} \mathcal{G} \simeq$ $R \operatorname{Hom}\left(\mathcal{F}_{x}, I_{x}\right)$, but since $I_{x}$ is injective, this is simply $\mathcal{H}$ om $\left(\mathcal{F}_{x}, I_{x}\right)$. Since $R \Gamma_{x} \mathcal{G}$ is concentrated in degree 0 , there is an open subscheme $U \subset X$ such that $\left.\mathcal{G}\right|_{U}$ is a sheaf. Replacing $U$ by a smaller open subscheme if necessary, we may assume that $\left.\omega_{X}\right|_{U} \in \mathcal{C}_{G}(U)_{\geq \text {alt } X}$. By Remark 3.2, we then know that $\left.\mathcal{G}\right|_{U} \simeq \mathcal{H}$ om $\left(\left.\mathcal{F}\right|_{U},\left.\omega_{X}\right|_{U}\right)$ lies in $\mathcal{C}_{G}(U) \geq$ alt $X-w$.

Now, consider the sequence

$$
\left.\left.\Gamma_{x} \sigma_{\leq \text {alt } X-w} \mathcal{G}\right|_{U} \subset \Gamma_{x} \sigma_{\leq \text {alt } X-w+1} \mathcal{G}\right|_{U} \subset \cdots
$$

This sequence is eventually constant; let $N \geq$ alt $X-w$ be the smallest integer such that

$$
\left.\Gamma_{x} \sigma_{\leq N} \mathcal{G}\right|_{U}=\left.\Gamma_{x} \sigma_{\leq v} \mathcal{G}\right|_{U}
$$

for all $v \geq N$. By replacing $U$ by a smaller open subscheme if necessary, we may assume that $\left.\sigma_{\geq N+1} \mathcal{G}\right|_{U}=0$, but $\left.\sigma_{\geq N} \mathcal{G}\right|_{V} \neq 0$ for all open subschemes $V \subset U$.

If $N=$ alt $X-w$, we are done, so assume $N>$ alt $X-w$. Now, form the exact sequence

$$
\left.\left.\left.0 \rightarrow \sigma_{\leq N-1} \mathcal{G}\right|_{U} \rightarrow \mathcal{G}\right|_{U} \rightarrow \sigma_{\geq N} \mathcal{G}\right|_{U} \rightarrow 0
$$

By invoking Lemma 6.6 three times and replacing $U$ by a smaller open subscheme as necessary, we can assume that the duality functor takes each term of the above 
sequence to a sheaf. (Note that $N$ has been chosen in such a way that $\left.\sigma_{\geq N} \mathcal{G}\right|_{U}$ remains nonzero as we shrink $U$.) Thus, $\mathbb{D}$ transforms the exact sequence above into

$$
\left.\left.\left.0 \rightarrow \mathbb{D}\left(\sigma_{\geq N} \mathcal{G}\right)\right|_{U} \rightarrow \mathcal{F}\right|_{U} \rightarrow \mathbb{D}\left(\sigma_{\leq N-1} \mathcal{G}\right)\right|_{U} \rightarrow 0
$$

Now, $\left.\mathbb{D}\left(\sigma_{>N} \mathcal{G}\right)\right|_{U}$ is a subsheaf of $\left.\mathcal{F}\right|_{U}$, so clearly $\left.\mathbb{D}\left(\sigma_{>N} \mathcal{G}\right)\right|_{U} \in \mathcal{C}_{G}(U)_{>w}$. By axioms ( $\mathrm{S7}$ ) and ( $\mathrm{S} 8$ ), we may replace $U$ by a smaller open subscheme and assume that the tensor product $\left.\left.\mathbb{D}\left(\sigma_{\geq N} \mathcal{G}\right)\right|_{U} \otimes \sigma_{\geq N} \mathcal{G}\right|_{U} \simeq \mathcal{H}$ om $\left.\left(\left.\sigma_{\geq N} \mathcal{G}\right|_{U},\left.\omega_{X}\right|_{U}\right) \otimes \sigma_{\geq N} \mathcal{G}\right|_{U}$ lies in $\mathcal{C}_{G}(U)_{\geq w+N}$, as does the image of the evaluation morphism

$$
\mathrm{ev}:\left.\left.\mathcal{H o m}\left(\left.\sigma_{\geq N} \mathcal{G}\right|_{U},\left.\omega_{X}\right|_{U}\right) \otimes \sigma_{\geq N} \mathcal{G}\right|_{U} \rightarrow \omega_{X}\right|_{U} .
$$

By assumption, $w+N>$ alt $X$, so $\left.\omega_{X}\right|_{U}$ contains the direct sum $\left.\sigma_{\leq \text {alt } X} \omega_{X}\right|_{U} \oplus$ $\operatorname{im}(\mathrm{ev})$. By Lemma 6.4 we can replace $U$ by a smaller subscheme and assume that $\operatorname{im}(\mathrm{ev})=0$ (clearly, $\left.\sigma_{\leq \text {alt } X} \omega_{X}\right|_{U}$ cannot be 0$)$. But ev $=0$ implies that $\mathcal{H} o m\left(\left.\sigma_{\geq N} \mathcal{G}\right|_{U},\left.\omega_{X}\right|_{U}\right)=0$, and $\left.\mathbb{D}\left(\sigma_{\geq N} \mathcal{G}\right)\right|_{U}=0$ in turn implies that $\left.\sigma_{\geq N} \mathcal{G}\right|_{U}=$ 0 . This is a contradiction, so we conclude that $N=$ alt $X-w$, and $\left.\mathcal{G}\right|_{U} \in$ $\mathcal{C}_{G}(U)_{\leq \text {alt } X-w} \cap \mathcal{C}_{G}(U)_{\geq \text {alt } X-w}$.

Proposition 6.8. Assume that $X$ is irreducible and that $\omega_{X}$ is a sheaf. Let $\mathcal{F} \in$ $\mathcal{C}_{G}(X)_{\geq w}$. Then there is an open subscheme $U \subset X$ such that $\left.\mathbb{D}(\mathcal{F})\right|_{U}$ is a sheaf in $\mathcal{C}_{G}(U)_{\leq \text {alt } X-w}$.

Proof. By axiom (S5), there is a $v \in \mathbb{Z}$ such that $\mathcal{F} \in \mathcal{C}_{G}(X)_{\leq v}$. Obviously, we have $v \geq w$. We proceed by induction on $v-w$. If $v-w=0$, then we are in the situation of Lemma 6.7 so the statement is already known. Otherwise, consider the short exact sequence

$$
0 \rightarrow \sigma_{\leq w} \mathcal{F} \rightarrow \mathcal{F} \rightarrow \sigma_{\geq w+1} \mathcal{F} \rightarrow 0 .
$$

Apply Lemma 6.6 to each term of this sequence: let $U$ be an open subscheme on which $\mathbb{D}$ takes all three terms to sheaves. Thus, the long exact sequence

$0 \rightarrow H^{0}\left(\mathbb{D}\left(\sigma_{\geq w+1} \mathcal{F}\right)\right) \rightarrow H^{0}(\mathbb{D}(\mathcal{F})) \rightarrow H^{0}\left(\mathbb{D}\left(\sigma_{\leq w} \mathcal{F}\right)\right) \rightarrow H^{1}\left(\mathbb{D}\left(\sigma_{\geq w+1} \mathcal{F}\right)\right) \rightarrow \cdots$,

when restricted to $U$, gives to the short exact sequence

$$
\left.\left.\left.0 \rightarrow \mathbb{D}\left(\sigma_{\geq w+1} \mathcal{F}\right)\right|_{U} \rightarrow \mathbb{D}(\mathcal{F})\right|_{U} \rightarrow \mathbb{D}\left(\sigma_{\leq w} \mathcal{F}\right)\right|_{U} \rightarrow 0 .
$$

Now, by the inductive assumption, we can replace $U$ by a smaller open subscheme so that the first term lies in $\mathcal{C}_{G}(U)_{\leq \text {alt } X-w-1}$, and by Lemma 6.7, we can similarly assume that the last term lies in $\mathcal{C}_{G}(U)_{<\text {alt } X-w}$. Since $\mathcal{C}_{G}(U)_{<\text {alt } X-w}$ is stable under extensions, we have $\left.\mathbb{D}(\mathcal{F})\right|_{U} \in \mathcal{C}_{G}(U)_{\leq a l t} X-w$, as desired.

\section{The Staggered $t$-Structure: Orthogonality}

In this section, we define the subcategories of $\mathcal{D}_{G}^{\mathrm{b}}(X)$ that will constitute the staggered $t$-structure, and we establish a number of their basic properties. The proof that they actually give a $t$-structure will be completed in the next section.

Let $X^{\text {gen }}$ be the set of generic points of ( $G$-invariant) subschemes of $X$. For each $x \in X^{\text {gen }}$, let $\overline{G x}$ denote the smallest ( $G$-invariant) closed subset of $X$ containing $x$. ( $\overline{G x}$ is not endowed with a fixed subscheme structure.) We continue to assume, as in the previous section, that $X$ is endowed with an $s$-structure.

Let us fix, once and for all, an equivariant dualizing complex $\omega_{X}$ on $X$. For each $x \in X^{\text {gen }}$, let $\operatorname{cod} \overline{G x}$ be the degree in which the complex $R \Gamma_{x}\left(\omega_{X}\right)$ is concentrated. 
This is a "codimension function" in the sense of $[\mathrm{H}, \S \mathrm{V} .7]$ : for any other point $y \in X^{\text {gen }}$, we have

$$
\operatorname{cod} \overline{G x}-\operatorname{cod} \overline{G y}=\operatorname{dim} \overline{G y}-\operatorname{dim} \overline{G x} .
$$

(In B1], $\omega_{X}$ was assumed to be normalized so that $\operatorname{cod} \overline{G x}=-\operatorname{dim} \overline{G x}$, but we do not make that assumption.) More generally, for any closed subscheme $Z \subset X$, we define

$$
\operatorname{cod} Z=\min \left\{\operatorname{cod} \overline{G x} \mid x \in Z^{\text {gen }}\right\} .
$$

If $j: U \hookrightarrow X$ is an open subscheme, then $j^{*} \omega_{X}$ is a dualizing complex on $U$, and if $i: Z \hookrightarrow X$ is a closed subscheme, then $R i^{!} \omega_{X}$ is a dualizing complex on $Z$. Thus, the choice of $\omega_{X}$ determines a choice of dualizing complex on all locally closed subschemes of $X$. The notation $\mathbb{D}$, when applied to complexes of sheaves on a subscheme of $X$, should be understood to be defined with respect to the dualizing complex obtained from $\omega_{X}$ in this way.

Definition 7.1. The staggered codimension of an irreducible closed $G$-invariant subscheme $Y \subset X$, denoted $\operatorname{scod} Y$, is defined to be $\operatorname{cod} Y+\operatorname{alt} Y$.

Definition 7.2. A perversity is a function $p: X^{\text {gen }} \rightarrow \mathbb{Z}$ such that for any $x, y \in$ $X^{\text {gen }}$ with $x \in \overline{G y}$, we have

$$
\begin{aligned}
& p(x) & \geq p(y) \\
\text { (monotonicity) } & \operatorname{scod} \overline{G x}-p(x) & \geq \operatorname{scod} \overline{G y}-p(y)
\end{aligned}
$$

If $p$ is a perversity, then the function $\bar{p}: X^{\text {gen }} \rightarrow \mathbb{Z}$ defined by

$$
\bar{p}(x)=\operatorname{scod} \overline{G x}-p(x)
$$

is clearly also a perversity. It is called the dual perversity to $p$.

Note that if $y$ is another generic point of $\overline{G x}$, then the monotonicity condition implies that $p(x)=p(y)$.

Define two full subcategories of $\mathcal{D}_{G}^{-}(X)$ as follows:

$$
\left.\begin{array}{l}
\mathcal{D}_{G}^{-}(X)_{\diamond}^{\leq n}=\left\{\mathcal{F} \in \mathcal{D}_{G}^{-}(X) \mid \text { for all } k \in \mathbb{Z}, H^{k}(\mathcal{F}) \in \mathcal{C}_{G}(X)_{\leq n-k}\right\} \\
\mathcal{D}_{G}^{-}(X)_{\mathbf{\Lambda}}^{\leq n}=\left\{\mathcal{F} \in \mathcal{D}_{G}^{-}(X) \mid \begin{array}{c}
\text { for all } k \in \mathbb{Z}, \text { there is a dense open subscheme } \\
V \Subset X \text { such that }\left.H^{k}(\mathcal{F})\right|_{V} \in \mathcal{C}_{G}(V)_{\leq n-k}
\end{array}\right.
\end{array}\right\}
$$

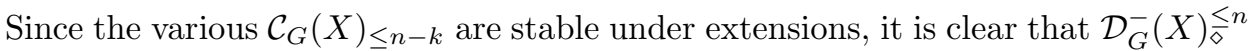
and $\mathcal{D}_{G}^{-}(X)_{\mathbf{\Lambda}}^{\leq n}$ are stable under extensions as well. It is also clear that both of these categories are stable under all truncation functors (with respect to the standard $t$ structure), since they are defined by conditions on cohomology sheaves.

Remark 7.3. The notion of "staggered degree" that was informally introduced in Section 1 can be formalized as follows: an object $\mathcal{F} \in \mathcal{D}_{G}^{\mathrm{b}}(X)$ is said to be concentrated in staggered degree $n$ if for all $k \in \mathbb{Z}$, the sheaf $H^{k}(\mathcal{F})$ is pure of step $n-k$. This notion is not useful for the general developments of this section or the following one, but it will come up when we study simple objects in Section 9 ,

Given a perversity function $p$, define a full subcategory of $\mathcal{D}_{G}^{-}(X)$ by

$$
{ }^{p} \mathcal{D}_{G}^{-}(X)^{\leq 0}=\left\{\begin{array}{l|l}
\mathcal{F} & \begin{array}{c}
\text { for any } x \in X^{\text {gen }} \text { and any closed } \\
\text { subscheme structure } i: Z \hookrightarrow X \\
\text { on } \overline{G x}, \text { we have } L i^{*} \mathcal{F} \in \mathcal{D}_{G}^{-}(Z)_{\mathbf{\Delta}}^{\leq p(x)}
\end{array}
\end{array}\right\} .
$$


Next, we define a full subcategory of $\mathcal{D}_{G}^{+}(X)$ by

$$
{ }^{p} \mathcal{D}_{G}^{+}(X)^{\geq 0}=\mathbb{D}\left({ }^{\bar{p}} \mathcal{D}_{G}^{-}(X)^{\leq 0}\right) .
$$

The fact that the various $\mathcal{D}_{G}^{-}(Z)^{\leq p(x)}$ are stable under extensions implies that ${ }^{p} \mathcal{D}_{G}^{-}(X) \leq 0$ and ${ }^{p} \mathcal{D}_{G}^{+}(X) \geq 0$ are as well. We also define

$$
{ }^{p} \mathcal{D}_{G}^{-}(X)^{\leq n}={ }^{p} \mathcal{D}_{G}^{-}(X)^{\leq 0}[-n] \quad \text { and } \quad{ }^{p} \mathcal{D}_{G}^{+}(X)^{\geq n}={ }^{p} \mathcal{D}_{G}^{+}(X)^{\geq 0}[-n],
$$

as well as

$$
{ }^{p} \mathcal{D}_{G}^{\mathrm{b}}(X)^{\leq n}={ }^{p} \mathcal{D}_{G}^{-}(X)^{\leq n} \cap \mathcal{D}_{G}^{\mathrm{b}}(X) \quad \text { and } \quad{ }^{p} \mathcal{D}_{G}^{\mathrm{b}}(X)^{\geq n}={ }^{p} \mathcal{D}_{G}^{+}(X)^{\geq n} \cap \mathcal{D}_{G}^{\mathrm{b}}(X) .
$$

The rest of this section and the following one will be devoted to the proof of the following theorem, which is one of the main results of the paper.

Theorem 7.4. $\left({ }^{p} \mathcal{D}_{G}^{\mathrm{b}}(X)^{\leq 0},{ }^{p} \mathcal{D}_{G}^{\mathrm{b}}(X)^{\geq 0}\right)$ is a nondegenerate, bounded $t$-structure on $\mathcal{D}_{G}^{\mathrm{b}}(X)$.

Definition 7.5. The $t$-structure $\left({ }^{p} \mathcal{D}_{G}^{\mathrm{b}}(X) \leq 0,{ }^{p} \mathcal{D}_{G}^{\mathrm{b}}(X)^{\geq 0}\right)$ is called the staggered $t$ structure on $\mathcal{D}_{G}^{\mathrm{b}}(X)$. Its heart, denoted ${ }^{p} \mathcal{M}(X)$, is the category of staggered sheaves.

Let $Y \subset X$ be a locally closed subscheme. Any perversity $p: X^{\text {gen }} \rightarrow \mathbb{Z}$ determines, by restriction, a perversity $Y^{\text {gen }} \rightarrow \mathbb{Z}$ as well. By an abuse of notation, we denote the latter by $p$ as well. Then, the categories ${ }^{p} \mathcal{D}_{G}^{-}(Y) \leq 0$ and ${ }^{p} \mathcal{D}_{G}^{+}(Y)^{\geq 0}$ are defined just as in (7.1)-(7.2).

Lemma 7.6. (1) Let $j: U \hookrightarrow X$ be an open subscheme. The functor $j^{*}$ takes ${ }^{p} \mathcal{D}_{G}^{-}(X) \leq 0$ to ${ }^{p} \mathcal{D}_{G}^{-}(U) \leq 0$, and ${ }^{p} \mathcal{D}_{G}^{+}(X)^{\geq 0}$ to ${ }^{p} \mathcal{D}_{G}^{+}(U)^{\geq 0}$.

(2) Let $i: Z \hookrightarrow X$ be a closed subscheme. Then $L i^{*}$ takes ${ }^{p} \mathcal{D}_{G}^{-}(X) \leq 0$ to ${ }^{p} \mathcal{D}_{G}^{-}(Z) \leq 0$, and $R i^{!}$takes ${ }^{p} \mathcal{D}_{G}^{+}(X)^{\geq 0}$ to ${ }^{p} \mathcal{D}_{G}^{+}(Z)^{\geq 0}$.

(3) Let $i: Z \hookrightarrow X$ be a closed subscheme. Then $i_{*}$ takes $\mathcal{D}_{G}^{-}(Z)_{\diamond}^{\leq n}$ to $\mathcal{D}_{G}^{-}(X)_{\diamond}^{\leq n}$.

Corollary 8.5 is another statement that is similar in spirit to this lemma, but we will be able to prove after establishing Theorem 7.4 .

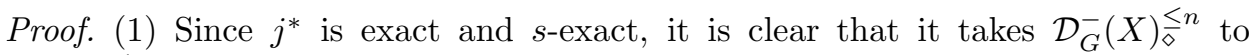
$\mathcal{D}_{G}^{-}(U)^{\leq n}$. It follows that $j^{*}$ takes ${ }^{p} \mathcal{D}_{G}^{-}(X) \leq 0$ to ${ }^{p} \mathcal{D}_{G}^{-}(U) \leq 0$. Because $\mathbb{D}$ commutes with $j^{*}$, we see that $j^{*}$ takes ${ }^{p} \mathcal{D}_{G}^{+}(X)^{\geq 0}$ to ${ }^{p} \mathcal{D}_{G}^{+}(U)^{\geq 0}$ as well.

(2) It is immediate from the definition of ${ }^{p} \mathcal{D}_{G}^{-}(X) \leq 0$ that $L i^{*}$ takes ${ }^{p} \mathcal{D}_{G}^{-}(X) \leq 0$ to ${ }^{p} \mathcal{D}_{G}^{-}(Z) \leq 0$. We obtain the statement on $R i^{!}$from the fact that $\mathbb{D} \circ L i^{*} \simeq R i^{!} \circ \mathbb{D}$.

(3) This follows from the fact that $i_{*}$ is exact and $s$-exact.

The following lemma is an immediate consequence of Lemma 6.1.

Lemma 7.7. For any $x \in X^{\text {gen }}$ and any closed subscheme structure $i: Z \hookrightarrow X$ on $\overline{G x}$, there is an open subscheme $V \subset Z$ such that $\left.R i^{!} \omega_{X}\right|_{V}$ is concentrated in degree $\operatorname{cod} \overline{G x}$.

Lemma 7.8. If $\mathcal{F} \in{ }^{p} \mathcal{D}_{G}^{-}(X) \leq 0$, then for any $n \in \mathbb{Z}$, we have $\tau^{\geq n} \mathcal{F} \in{ }^{p} \mathcal{D}_{G}^{\mathrm{b}}(X) \leq 0$ and $\tau \leq n \mathcal{F} \in{ }^{P} \mathcal{D}_{G}^{-}(X) \leq 0$.

This really is a one-sided statement: in general, ${ }^{p} \mathcal{D}_{G}^{+}(X)^{\geq 0}$ is not stable under standard truncation functors. 
Proof. Let $a$ be the largest integer such that $H^{a}(\mathcal{F}) \neq 0$, and form the distinguished triangle

$$
\tau^{\leq a-1} \mathcal{F} \rightarrow \mathcal{F} \rightarrow \tau^{\geq a} \mathcal{F} \rightarrow
$$

Let $\mathcal{F}_{0}=H^{a}(\mathcal{F})$, so that $\tau^{\geq a} \mathcal{F} \simeq \mathcal{F}_{0}[-a]$. For any point $x \in X^{\text {gen }}$ and any subscheme structure $i: Z \hookrightarrow X$ on $\overline{G x}$, this gives rise to a distinguished triangle

$$
L i^{*} \tau^{\leq a-1} \mathcal{F} \rightarrow L i^{*} \mathcal{F} \rightarrow L i^{*} \mathcal{F}_{0}[-a] \rightarrow .
$$

Since the first term belongs to $\mathcal{D}_{G}^{-}(Z) \leq a-1$, we see from the long exact cohomology sequence that $H^{a}\left(L i^{*} \mathcal{F}\right) \simeq H^{a}\left(L i^{*} \mathcal{F}_{0}[-a]\right) \simeq H^{0}\left(L i^{*} \mathcal{F}_{0}\right) \simeq i^{*} \mathcal{F}_{0}$. Next, from the definition of ${ }^{p} \mathcal{D}_{G}^{-}(X) \leq 0$, we know that there is an open subscheme $V \Subset Z$ such that $\left.i^{*} \mathcal{F}_{0}\right|_{V} \in \mathcal{C}_{G}(V)_{\leq p(x)-a}$. Let $U \Subset X$ be any dense open subscheme such that $U \cap Z=V$. Then $\left.\mathcal{F}_{0}\right|_{U} \in \mathcal{C}_{G}^{\text {loc }}(U, V)_{\leq p(x)-a}$. By Lemma 3.10, for any $k \geq 0$, we can find another open set $V^{\prime} \Subset V$ such that $\left.H^{-k}\left(L i^{*} \mathcal{F}_{0}\right)\right|_{V^{\prime}} \in \mathcal{C}_{G}\left(V^{\prime}\right)_{\leq p(x)-a} \subset$ $\mathcal{C}_{G}\left(V^{\prime}\right)_{\leq p(x)+k-a}$, or, equivalently, $\left.H^{a-k}\left(L i^{*} \mathcal{F}_{0}[-a]\right)\right|_{V^{\prime}} \in \mathcal{C}_{G}\left(V^{\prime}\right)_{\leq p(x)+k-a}$. We have thus shown that $\mathcal{F}_{0}[-a] \in{ }^{p} \mathcal{D}_{G}^{\mathrm{b}}(X) \leq 0$.

Next, for any $k \geq 1$, consider the following exact sequence:

$$
H^{a-k-1}\left(L i^{*} \mathcal{F}_{0}[-a]\right) \rightarrow H^{a-k}\left(L i^{*} \tau^{\leq a-1} \mathcal{F}\right) \rightarrow H^{a-k}\left(L i^{*} \mathcal{F}\right) .
$$

Upon restriction to a suitable open subscheme $V \Subset Z$, we know that the last term yields an object in $\mathcal{C}_{G}(V)_{<p(x)+k-a}$, and the first term yields an object of $\mathcal{C}_{G}(V)_{\leq p(x)-a} \subset \mathcal{C}_{G}(V)_{\leq p(x)+k-a}$. Therefore, we have that $\left.H^{a-k}\left(L i^{*} \tau^{\leq a-1} \mathcal{F}\right)\right|_{V} \in$ $\mathcal{C}_{G}(V)_{\leq p(x)+k-a}$ as well. This shows that that $\tau^{\leq a-1} \mathcal{F} \in{ }^{p} \mathcal{D}_{G}^{-}(X) \leq 0$.

We have seen that all three terms of (7.3) lie in ${ }^{p} \mathcal{D}_{G}^{-}(X) \leq 0$. Applying this result to $\tau^{\leq a-1} \mathcal{F}$ yields in particular that $\tau^{\geq a-1}\left(\tau^{\leq a-1} \mathcal{F}\right) \simeq H^{a-1}(\mathcal{F})[1-a] \in{ }^{p} \mathcal{D}_{G}^{-}(X) \leq 0$. Indeed, by induction, one finds that for all $k$, we have $\tau^{\leq k} \mathcal{F} \in{ }^{{ }^{P}} \mathcal{D}_{G}^{-}(X) \leq 0$ and $H^{k}(\mathcal{F})[-k] \in{ }^{\mathcal{D}} \mathcal{D}_{G}^{\mathrm{b}}(X) \leq 0$. By a further induction argument, using the fact that ${ }^{p} \mathcal{D}_{G}^{\mathrm{b}}(X) \leq 0$ is stable extensions, the latter fact implies that $\tau^{\geq k} \mathcal{F} \in{ }^{p} \mathcal{D}_{G}^{\mathrm{b}}(X) \leq 0$, as desired.

Proposition 7.9. Suppose $\mathcal{F} \in{ }^{p} \mathcal{D}_{G}^{-}(X)^{\leq 0}$ and $\mathcal{G} \in{ }^{p} \mathcal{D}_{G}^{+}(X)^{\geq 1}$. Then we have $\operatorname{Hom}(\mathcal{F}, \mathcal{G})=0$.

Proof. Suppose $\mathcal{G} \in \mathcal{D}_{G}^{+}(X)^{\geq a}$. Then we have $\operatorname{Hom}(\mathcal{F}, \mathcal{G}) \simeq \operatorname{Hom}\left(\tau^{\geq a} \mathcal{F}, \mathcal{G}\right)$, and by the preceding lemma, $\tau^{\geq a} \mathcal{F} \in{ }^{p} \mathcal{D}_{G}^{\mathrm{b}}(X) \leq 0$. By replacing $\mathcal{F}$ by $\tau^{\geq a} \mathcal{F}$ if necessary, we may assume without loss of generality that $\mathcal{F}$ is actually a bounded complex.

By definition, there is an object $\mathcal{G}_{1} \in \bar{p} \mathcal{D}_{G}^{-}(X)^{\leq-1}$ such that $\mathbb{D}\left(\mathcal{G}_{1}\right) \simeq \mathcal{G}$. Now, suppose $\mathcal{F} \in \mathcal{D}_{G}^{-}(X)^{\leq b}$. Then, as above, we have $\operatorname{Hom}(\mathcal{F}, \mathcal{G}) \simeq \operatorname{Hom}\left(\mathcal{F}, \tau^{\leq b} \mathcal{G}\right)$. Consider applying the functor $\mathbb{D}$ to the distinguished triangle

$$
\tau^{\leq \operatorname{cod} X-b-1} \mathcal{G}_{1} \rightarrow \mathcal{G}_{1} \rightarrow \tau^{\geq \operatorname{cod} X-b} \mathcal{G}_{1} \rightarrow .
$$

Recall that $\omega_{X} \in \mathcal{D}_{G}^{+}(X)^{\geq \operatorname{cod} X}$. We therefore have

$$
\mathbb{D}\left(\tau^{\leq \operatorname{cod} X-b-1} \mathcal{G}_{1}\right) \simeq R \mathcal{H o m}\left(\tau^{\leq \operatorname{cod} X-b-1} \mathcal{G}_{1}, \omega_{X}\right) \in \mathcal{D}_{G}^{+}(X)^{\geq b+1} .
$$

In particular, it follows $\tau^{\leq b} \mathbb{D}\left(\tau^{\geq \operatorname{cod} X-b} \mathcal{G}_{1}\right) \simeq \tau^{\leq b} \mathbb{D}\left(\mathcal{G}_{1}\right) \simeq \tau^{\leq b} \mathcal{G}$. That is, we may replace $\mathcal{G}_{1}$ by $\tau^{\geq \operatorname{cod} X-b} \mathcal{G}_{1}$ without affecting the calculation of $\operatorname{Hom}(\mathcal{F}, \mathcal{G})$. Thus, we henceforth assume that $\mathcal{G}_{1}$ is a bounded complex as well. 
Choose a generic point $x \in X$, and let $U \subset X$ be an irreducible open subscheme containing $x$ such that

$$
\left.\mathcal{F}\right|_{U} \in \mathcal{D}_{G}^{-}(U)_{\mathbf{\Lambda}}^{\leq p(x)} \quad \text { and }\left.\quad \mathcal{G}_{1}\right|_{U} \in \mathcal{D}_{G}^{-}(U)_{\mathbf{\Lambda}}^{\leq \bar{p}(x)-1} .
$$

We will first show by induction on the number of nonzero cohomology sheaves of $\mathcal{F}$ and $\mathcal{G}_{1}$ that, possibly after replacing $U$ by a smaller open subscheme, we may assume that $\operatorname{Hom}\left(\left.\mathcal{F}\right|_{U},\left.\mathcal{G}\right|_{U}\right)=0$.

Assume for the moment that $\mathcal{F}$ and $\mathcal{G}_{1}$ are each actually concentrated in a single degree, say in degrees $a$ and $b$, respectively. Then $\mathcal{F} \simeq \mathcal{F}^{\prime}[-a]$ and $\mathcal{G}_{1} \simeq \mathcal{G}_{1}^{\prime}[-b]$ for some sheaves $\mathcal{F}^{\prime}, \mathcal{G}_{1}^{\prime} \in \mathcal{C}_{G}(X)$. Replacing $U$ be a smaller open subscheme if necessary, we may assume that $\left.\left.H^{a}(\mathcal{F})\right|_{U} \simeq \mathcal{F}^{\prime}\right|_{U} \in \mathcal{C}_{G}(U)_{\leq p(x)-a}$ and $\left.H^{b}(\mathcal{G})\right|_{U} \simeq$ $\left.\mathcal{G}_{1}^{\prime}\right|_{U} \in \mathcal{C}_{G}(U)_{\leq \bar{p}(x)-1-b}$. By Lemma 6.7, we may further assume, after perhaps replacing $U$ by a yet smaller subscheme, that $\left.\mathbb{D}\left(\mathcal{G}_{1}\right)\right|_{U}$ is concentrated in a single degree. That degree must be $\operatorname{cod} \overline{G x}-b$. Thus, on $U$, we have

$$
\left.\mathbb{D}\left(\mathcal{G}_{1}\right)\right|_{U} \simeq R \mathcal{H o m}\left(\mathcal{G}_{1}^{\prime}[-b],\left.\omega_{X}\right|_{U}\right) \simeq \mathcal{H o m}\left(\left.\mathcal{G}_{1}^{\prime}\right|_{U},\left.\omega_{X}[\operatorname{cod} \overline{G x}]\right|_{U}\right)[-\operatorname{cod} \overline{G x}+b] .
$$

Now,

$$
\begin{aligned}
\operatorname{Hom}\left(\left.\mathcal{F}\right|_{U},\left.\mathcal{G}\right|_{U}\right) & \simeq \operatorname{Hom}\left(\left.\mathcal{F}^{\prime}[-a]\right|_{U},\left.\mathcal{D}\left(\mathcal{G}_{1}\right)\right|_{U}\right) \\
& \simeq \operatorname{Hom}\left(\left.\mathcal{F}^{\prime}[-a]\right|_{U}, \mathcal{H} \text { om }\left(\left.\mathcal{G}_{1}^{\prime}\right|_{U},\left.\omega_{X}[\operatorname{cod} \overline{G x}]\right|_{U}\right)[-\operatorname{cod} \overline{G x}+b]\right) \\
& \simeq \operatorname{Ext}^{-\operatorname{cod} X+a+b}\left(\left.\mathcal{F}^{\prime}\right|_{U}, \mathcal{H} o m\left(\left.\mathcal{G}_{1}^{\prime}\right|_{U},\left.\omega_{X}[\operatorname{cod} \overline{G x}]\right|_{U}\right)\right) .
\end{aligned}
$$

If $-\operatorname{cod} \overline{G x}+a+b<0$, then we evidently have $\operatorname{Hom}\left(\left.\mathcal{F}\right|_{U},\left.\mathcal{G}\right|_{U}\right)=0$. Assume instead now that $-\operatorname{cod} \overline{G x}+a+b \geq 0$. Next, by replacing $U$ by a smaller open subscheme if necessary, we may assume that $\left.\omega_{X}[\operatorname{cod} \overline{G x}]\right|_{U} \in \mathcal{C}_{G}(U)_{\geq \text {alt }} \overline{G x}$ (see Definition 6.3). Since $\left.\mathcal{G}_{1}^{\prime}\right|_{U} \in \mathcal{C}_{G}(U)_{\leq \bar{p}(x)-1-b}$, we see that

$$
\begin{aligned}
\mathcal{H o m}\left(\left.\mathcal{G}_{1}^{\prime}\right|_{U},\left.\omega_{X}[\operatorname{cod} \overline{G x}]\right|_{U}\right) \in & \mathcal{C}_{G}(U)_{\geq \operatorname{alt}} \overline{G x}-\bar{p}(x)+1+b \\
& =\mathcal{C}_{G}(U)_{\geq-\operatorname{cod} \overline{G x}+p(x)+b+1} \subset \mathcal{C}_{G}(U)_{\geq-a+p(x)+1},
\end{aligned}
$$

where the last inclusion comes from the fact that $-\operatorname{cod} \overline{G x}+b \geq-a$. Since $\left.\mathcal{F}^{\prime}\right|_{U} \in$ $\mathcal{C}_{G}(U)_{\leq p(x)-a}$, we may invoke axiom (\$9): after replacing $U$ by a smaller open subscheme, we may assume that

$$
\operatorname{Ext}^{-\operatorname{cod} \overline{G x}+a+b}\left(\left.\mathcal{F}^{\prime}\right|_{U}, \mathcal{H o m}\left(\left.\mathcal{G}_{1}^{\prime}\right|_{U},\left.\omega_{X}[\operatorname{cod} \overline{G x}]\right|_{U}\right)\right)=0,
$$

and hence that $\operatorname{Hom}\left(\left.\mathcal{F}\right|_{U},\left.\mathcal{G}\right|_{U}\right)=0$, as desired.

Now, we return to the case where $\mathcal{F}$ is a bounded complex, not necessarily concentrated in a single degree. $\mathcal{G}_{1}$ is still assumed to be concentrated in degree $b$. Choose $U$ afresh as we originally did: it should be an irreducible open subscheme containing $x$ and satisfying the conditions (7.4). Any distinguished triangle of the form

$$
\left.\left.\left.\tau^{\leq k} \mathcal{F}\right|_{U} \rightarrow \mathcal{F}\right|_{U} \rightarrow \tau^{\geq k+1} \mathcal{F}\right|_{U} \rightarrow
$$

gives rise to a sequence

$$
\operatorname{Hom}\left(\left.\tau^{\geq k+1} \mathcal{F}\right|_{U},\left.\mathcal{G}\right|_{U}\right) \rightarrow \operatorname{Hom}\left(\left.\mathcal{F}\right|_{U},\left.\mathcal{G}\right|_{U}\right) \rightarrow \operatorname{Hom}\left(\left.\tau^{\leq k} \mathcal{F}\right|_{U},\left.\mathcal{G}\right|_{U}\right)
$$

that is exact at the middle term. The categories $\mathcal{D}_{G}^{-}(U)_{\mathbf{\Delta}}^{\leq p(x)}$ and $\mathcal{D}_{G}^{-}(U)^{\leq \overline{\boldsymbol{p}}}(x)-1$ are stable under all truncation functors: we have $\left.\tau^{\geq k+1} \mathcal{F}\right|_{U} \in \mathcal{D}_{G}^{-}(U)_{\mathbf{\Lambda}}^{\leq p(x)}$ and $\left.\tau^{\leq k} \mathcal{F}\right|_{U} \in \mathcal{D}_{G}^{-}(U)_{\mathbf{\Lambda}}^{\leq p(x)}$. By choosing $k$ such that $\tau^{\geq k+1} \mathcal{F}$ and $\tau^{\leq k} \mathcal{F}$ each have fewer nonzero cohomology sheaves than $\mathcal{F}$, we may assume inductively that $U$ has 
been chosen so that the first and last Hom-groups above vanish. Obviously, this implies that $\operatorname{Hom}\left(\left.\mathcal{F}\right|_{U},\left.\mathcal{G}\right|_{U}\right)=0$, as desired.

An analogous argument allows us to generalize to the case where $\mathcal{G}_{1}$ is a bounded complex not necessarily in a single degree.

Thus, in all cases, we have now constructed an open subscheme $U \subset X$ such that $\operatorname{Hom}\left(\left.\mathcal{F}\right|_{U},\left.\mathcal{G}\right|_{U}\right)=0$. Let $Z$ be a closed subscheme complementary to $U$, and consider the exact sequence ( $c f$. Lemma 2.1(3)

$$
\lim _{\overrightarrow{Z^{\prime}}} \operatorname{Hom}\left(L i_{Z^{\prime}}^{*} \mathcal{F}, R i_{Z^{\prime}}^{!} \mathcal{G}\right) \rightarrow \operatorname{Hom}(\mathcal{F}, \mathcal{G}) \rightarrow \operatorname{Hom}\left(\left.\mathcal{F}\right|_{U},\left.\mathcal{G}\right|_{U}\right) .
$$

We now know that the last term vanishes. The first term vanishes by the noetherian induction assumption, using Lemma 7.6. We conclude that $\operatorname{Hom}(\mathcal{F}, \mathcal{G})=0$, as desired.

\section{The Staggered $t$-Structure: Distinguished Triangles}

We will complete the proof of Theorem 7.4 in this section. We retain all the notation and assumptions of the preceding section.

Proposition 8.1. Suppose $\mathcal{F} \in \mathcal{D}_{G}^{\text {supp }}(X, Z) \cap \mathcal{D}_{G}^{-}(X)_{\diamond}^{\leq n}$. If $n \leq p(x)$ for all $x \in Z^{\text {gen }}$, then $\mathcal{F} \in{ }^{p} \mathcal{D}_{G}^{-}(X) \leq 0$.

Proof. Let $x \in X^{\text {gen }}$, and let $\kappa: Y \hookrightarrow X$ be a closed subscheme structure on $\overline{G x}$. We must show that $L \kappa^{*} \mathcal{F} \in \mathcal{D}_{G}^{-}(Y)^{\leq p(x)}$. If $x \notin Z$, this is trivially true: then $Y$ contains an open subscheme $V$ not meeting $Z$, necessarily dense since $Y$ is irreducible, and $\left.H^{k}\left(L \kappa^{*} \mathcal{F}\right)\right|_{V}=0$ for all $k$. Assume now that $x \in Z$. Since $\mathcal{F}$ is a bounded complex and ${ }^{p} \mathcal{D}_{G}^{-}(X) \leq 0$ is stable under extensions, we can use distinguished triangles of the form

$$
\tau^{\leq k} \mathcal{F} \rightarrow \mathcal{F} \rightarrow \tau^{\geq k+1} \mathcal{F} \rightarrow
$$

and induction on the number of nonzero cohomology sheaves of $\mathcal{F}$ to reduce to the case where $\mathcal{F}$ is concentrated in a single degree. (Here, we also use the fact that $\mathcal{D}_{G}^{-}(X)_{\bar{\diamond}}^{\leq n}$ and $\mathcal{D}_{G}^{\text {supp }}(X, Z)$ are stable under truncation functors. $)$

Suppose, then, that $\mathcal{F}$ is concentrated in degree $a$ : we have $\mathcal{F} \simeq \mathcal{F}_{1}[-a]$ for some sheaf $\mathcal{F}_{1} \in \mathcal{C}_{G}(X)_{\leq n-a}$. By Lemma 3.10, for all $k \leq a$, there is an open subscheme $V$ such that

$$
\left.\left.H^{k}\left(L \kappa^{*} \mathcal{F}\right)\right|_{V} \simeq H^{k-a}\left(L \kappa^{*} \mathcal{F}_{1}\right)\right|_{V} \in \mathcal{C}_{G}(V)_{\leq n-a} \subset \mathcal{C}_{G}(V)_{\leq p(x)-k},
$$

where we have made use of the assumption that $n \leq p(x)$. Of course, $H^{k}\left(L \kappa^{*} \mathcal{F}\right)=0$ if $k>a$. Thus, we see that $L \kappa^{*} \mathcal{F} \in \mathcal{D}_{G}^{-}(Y)_{\mathbf{\Lambda}}^{\leq p(x)}$, as desired.

Lemma 8.2. Let $U \subset X$ be an open subscheme, and let $Z \subset X$ be the complementary closed subscheme. Let $x$ be a generic point of $Z$, and let $\mathcal{F} \in \mathcal{D}_{G}^{\mathrm{b}}(X)^{[a, b]}$ be such that $\left.\mathcal{F}\right|_{U} \in \mathcal{D}_{G}^{\mathrm{b}}(U)_{\diamond}^{\leq n}$. Then there is an open subscheme $V \subset X$ with $U \subset V$ and $x \in V$, and a distinguished triangle

$$
\mathcal{F}^{\prime} \rightarrow \mathcal{F} \rightarrow \mathcal{F}^{\prime \prime} \rightarrow
$$

with $\mathcal{F}^{\prime} \in \mathcal{D}_{G}^{\mathrm{b}}(X)^{[a, b]},\left.\mathcal{F}^{\prime}\right|_{V} \in \mathcal{D}_{G}^{\mathrm{b}}(V)_{\diamond}^{\leq n}$, and $\mathcal{F}^{\prime \prime} \in \mathcal{D}_{G}^{\text {supp }}(X, Z)$. In addition, we have $\left.\mathbb{D}\left(\mathcal{F}^{\prime \prime}\right)\right|_{V} \in \mathcal{D}_{G}^{\mathrm{b}}(V)^{\leq d-n-1}$, where $d=\operatorname{scod} \overline{G x}$. 
Proof. We proceed by induction on $b-a$. (The base case $a=b$ will be treated as part of the general case.) Form the distinguished triangle

$$
\tau^{\leq a} \mathcal{F} \rightarrow \mathcal{F} \rightarrow \tau^{\geq a+1} \mathcal{F} \rightarrow
$$

Note that $\left.\tau^{\leq a} \mathcal{F}\right|_{U}$ and $\left.\tau^{\geq a+1} \mathcal{F}\right|_{U}$ both belong to $\mathcal{D}_{G}^{\mathrm{b}}(U)_{\Delta}^{\leq n}$, since that category is stable under truncation. Evidently, $\tau \leq a \mathcal{F} \in \mathcal{D}_{G}^{\mathrm{b}}(X)^{[a, a]}$. Let $\mathcal{F}_{1}=H^{a}(\mathcal{F}) \simeq$ $(\tau \leq a \mathcal{F})[a]$, and form the short exact sequence

$$
0 \rightarrow \sigma_{\leq n-a} \mathcal{F}_{1} \rightarrow \mathcal{F}_{1} \rightarrow \sigma_{\geq n-a+1} \mathcal{F}_{1} \rightarrow 0 .
$$

Now, the definition of $\mathcal{D}_{G}^{\mathrm{b}}(U)_{\bar{\diamond}}^{\leq n}$ implies that $\left.\mathcal{F}_{1}\right|_{U} \in \mathcal{C}_{G}(U)_{\leq n-a}$. It follows that $\left.\left(\sigma_{\geq n-a+1} \mathcal{F}_{1}\right)\right|_{U}=0$, or in other words, that $\sigma_{\geq n-a+1} \mathcal{F}_{1}$ is supported on $\underline{Z}$. Therefore, there is some subscheme structure $i_{Z^{\prime}}: Z^{\prime} \hookrightarrow X$ on $\underline{Z}$ and some sheaf $\mathcal{G}_{1} \in \mathcal{C}_{G}\left(Z^{\prime}\right)_{\geq n-a+1}$ such that $\sigma_{\geq n-a+1} \mathcal{F}_{1} \simeq i_{Z^{\prime} *} \mathcal{G}_{1}$. Recall that $x$ is a generic point of $Z$. There certainly exist open irreducible subschemes $V_{1}^{\prime} \subset Z^{\prime}$ containing $x$. By Proposition 6.8, we can choose a $V_{1}^{\prime}$ with the property that $\left.\mathbb{D}\left(\mathcal{G}_{1}\right)\right|_{V_{1}^{\prime}}$ is concentrated in degree $\operatorname{cod} \overline{G x}$, and such that the sheaf $\left.\mathbb{D}\left(\mathcal{G}_{1}\right)[\operatorname{cod} \overline{G x}]\right|_{V_{1}^{\prime}}$ is in $\mathcal{C}_{G}\left(V_{1}^{\prime}\right)_{\leq \text {alt }} \overline{G x}-(n-a+1)$.

Now, let $V^{\prime}=X \backslash\left(Z^{\prime} \backslash V_{1}^{\prime}\right)$. This is an open subscheme of $X$ containing $U$, and containing $V_{1}^{\prime}$ as a closed subscheme. Let $\mathcal{G}^{\prime}=\sigma_{\leq n-a} \mathcal{F}_{1}[-a]$, and let $\mathcal{G}^{\prime \prime}=\sigma_{\geq n-a+1} \mathcal{F}_{1}[-a] \simeq i_{Z^{\prime} *} \mathcal{G}_{1}[-a]$. The short exact sequence above gives rise to a distinguished triangle

$$
\mathcal{G}^{\prime} \rightarrow \tau^{\leq a} \mathcal{F} \rightarrow \mathcal{G}^{\prime \prime} \rightarrow,
$$

with $\mathcal{G}^{\prime} \in \mathcal{D}_{G}^{\mathrm{b}}(X)^{[a, a]},\left.\mathcal{G}^{\prime}\right|_{V^{\prime}} \in \mathcal{D}_{G}^{\mathrm{b}}\left(V^{\prime}\right)_{\diamond}^{\leq n}$ and $\mathcal{G}^{\prime \prime} \in \mathcal{D}_{G}^{\text {supp }}(X, Z)$. Moreover, the calculations above with $\mathcal{G}_{1}$ imply that $\left.\mathbb{D}\left(\mathcal{G}^{\prime \prime}\right)\right|_{V^{\prime}}$ is concentrated in degree $\operatorname{cod} \overline{G x}-a$, and that

$$
\begin{aligned}
H^{\operatorname{cod} \overline{G x}-a}\left(\left.\mathbb{D}\left(\mathcal{G}^{\prime \prime}\right)\right|_{V^{\prime}}\right) \in \mathcal{C}_{G}(V)_{\leq \operatorname{alt} \overline{G x}-(n-a+1)} & =\mathcal{C}_{G}(V)_{\leq(\operatorname{alt} \overline{G x}+\operatorname{cod} \overline{G x}-n-1)-(\operatorname{cod} \overline{G x}-a) .}
\end{aligned}
$$

Thus, $\left.\mathbb{D}\left(\mathcal{G}^{\prime \prime}\right)\right|_{V^{\prime}} \in \mathcal{D}_{G}^{\mathrm{b}}\left(V^{\prime}\right)^{\leq d-n-1}$, where $d=\operatorname{scod} \overline{G x}$. If $b=a$, then $\mathcal{F} \simeq \tau \leq a \mathcal{F}$, and we are finished: the distinguished triangle in (8.1) is the one we seek.

Now, suppose $b>a$. Then $\tau^{\geq a+1} \mathcal{F} \in \mathcal{D}_{G}^{\mathrm{b}}(X)^{[a+1, b]}$. By the inductive assumption, we can find an open subscheme $V^{\prime \prime} \subset X$ with $U \subset V^{\prime \prime}$ and $x \in V^{\prime \prime} \cap Z$, and a distinguished triangle

$$
\mathcal{H}^{\prime} \rightarrow \tau^{\geq a+1} \mathcal{F} \rightarrow \mathcal{H}^{\prime \prime} \rightarrow
$$

with $\mathcal{H}^{\prime} \in \mathcal{D}_{G}^{\mathrm{b}}(X)^{[a+1, b]},\left.\mathcal{H}^{\prime}\right|_{V^{\prime \prime}} \in \mathcal{D}_{G}^{\mathrm{b}}\left(V^{\prime \prime}\right) \frac{\leq n}{\triangleright}, \mathcal{H}^{\prime \prime} \in \mathcal{D}_{G}^{\text {supp }}(X, Z)$, and $\left.\mathbb{D}\left(\mathcal{H}^{\prime \prime}\right)\right|_{V^{\prime \prime}} \in$ $\mathcal{D}_{G}^{\mathrm{b}}\left(V^{\prime \prime}\right)_{\diamond}^{\leq d-n-1}$. Let $W=V^{\prime} \cap V^{\prime \prime}$. This is an open subscheme with $U \subset W$ and $x \in W$. Note that

$$
\left.\left.\mathcal{G}^{\prime \prime}[a]\right|_{W} \simeq i_{*} \mathcal{G}_{1}\right|_{W} \in \mathcal{C}_{G}^{\text {supp }}\left(W, W \cap V_{1}^{\prime}\right)_{\geq n-a+1} .
$$

On the other hand, we also have $\left.\mathcal{H}^{\prime}\right|_{W} \in \mathcal{D}_{G}^{\mathrm{b}}(W)_{\triangleright}^{\leq n}$, so whenever $a<k \leq b$, we have $\left.H^{k}\left(\mathcal{H}^{\prime}\right)\right|_{W} \in \mathcal{C}_{G}(W)_{\leq n-k} \subset \mathcal{C}_{G}(W)_{\leq n-a}$. Thus, by invoking axiom (S9) finitely many times, we may find a subscheme $V \Subset_{W \cap V_{1}^{\prime}} W$ such that

$$
\mathbf{E x t}^{1+k-a}\left(\left.H^{k}\left(\mathcal{H}^{\prime}\right)\right|_{V},\left.\mathcal{G}^{\prime \prime}[a]\right|_{V}\right)=0
$$

for all $k$ such that $a<k \leq b$. Note that $V \cap Z$ must contain $x$. 
We claim that $\operatorname{Hom}\left(\left.\mathcal{H}^{\prime}\right|_{V},\left.\mathcal{G}^{\prime \prime}[1]\right|_{V}\right)=0$. Indeed, we will show by induction on $k$ that $\operatorname{Hom}\left(\left.\tau^{\leq k} \mathcal{H}^{\prime}\right|_{V},\left.\mathcal{G}^{\prime \prime}\right|_{V}\right)=0$ for all $k$ with $a \leq k \leq b$. It is trivially true for $k=a$, since $\tau^{\leq a} \mathcal{H}^{\prime}=0$. Now, if $k \geq a+1$, form the distinguished triangle

$$
\tau^{\leq k-1} \mathcal{H}^{\prime} \rightarrow \tau^{\leq k} \mathcal{H}^{\prime} \rightarrow \tau^{[k, k]} \mathcal{H}^{\prime} \rightarrow
$$

This gives rise to a sequence

$$
\operatorname{Hom}\left(\left.\tau^{[k, k]} \mathcal{H}^{\prime}\right|_{V},\left.\mathcal{G}^{\prime \prime}[1]\right|_{V}\right) \rightarrow \operatorname{Hom}\left(\left.\tau^{\leq k} \mathcal{H}^{\prime}\right|_{V},\left.\mathcal{G}^{\prime \prime}[1]\right|_{V}\right) \rightarrow \operatorname{Hom}\left(\left.\tau^{\leq k-1} \mathcal{H}^{\prime}\right|_{V},\left.\mathcal{G}^{\prime \prime}[1]\right|_{V}\right)
$$

Here, the last term vanishes by assumption. On the other hand, we have $\tau^{[k, k]} \mathcal{H}^{\prime} \simeq$ $H^{k}\left(\mathcal{H}^{\prime}\right)[-k]$, and

$$
\operatorname{Hom}\left(\left.H^{k}\left(\mathcal{H}^{\prime}\right)[-k]\right|_{V},\left.\mathcal{G}^{\prime \prime}[1]\right|_{V}\right) \simeq \operatorname{Ext}^{1+k-a}\left(\left.H^{k}\left(\mathcal{H}^{\prime}\right)\right|_{V},\left.\mathcal{G}^{\prime \prime}[a]\right|_{V}\right)=0 .
$$

We now see from the sequence above that $\operatorname{Hom}\left(\tau \leq\left. k \mathcal{H}^{\prime}\right|_{V},\left.\mathcal{G}^{\prime \prime}[1]\right|_{V}\right)=0$. Since $\tau^{\leq b} \mathcal{H}^{\prime} \simeq \mathcal{H}^{\prime}$, we see that $\operatorname{Hom}\left(\left.\mathcal{H}^{\prime}\right|_{V},\left.\mathcal{G}^{\prime \prime}[1]\right|_{V}\right)=0$, as desired.

Next, consider the sequence

$$
\operatorname{Hom}\left(\left.\mathcal{H}^{\prime}\right|_{V},\left.\mathcal{G}^{\prime}[1]\right|_{V}\right) \rightarrow \operatorname{Hom}\left(\left.\mathcal{H}^{\prime}\right|_{V},\left.\left(\tau^{\leq a} \mathcal{F}\right)[1]\right|_{V}\right) \rightarrow \operatorname{Hom}\left(\left.\mathcal{H}^{\prime}\right|_{V},\left.\mathcal{G}^{\prime \prime}[1]\right|_{V}\right) .
$$

Since the last term vanishes, we see that every morphism $\left.\left.\mathcal{H}^{\prime}\right|_{V} \rightarrow(\tau \leq a \mathcal{F})[1]\right|_{V}$ must factor through the map $\left.\left.\mathcal{G}^{\prime}[1]\right|_{V} \rightarrow\left(\tau^{\leq a} \mathcal{F}\right)[1]\right|_{V}$. In particular, consider the composition

$$
\left.\left.\left.\mathcal{H}^{\prime}\right|_{V} \rightarrow \tau^{\geq a+1} \mathcal{F}\right|_{V} \rightarrow\left(\tau^{\leq a} \mathcal{F}\right)[1]\right|_{V}
$$

This gives rise to a commutative square

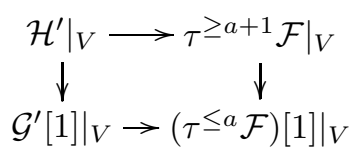

Let us complete this diagram using the 9-lemma, and then rearrange it:

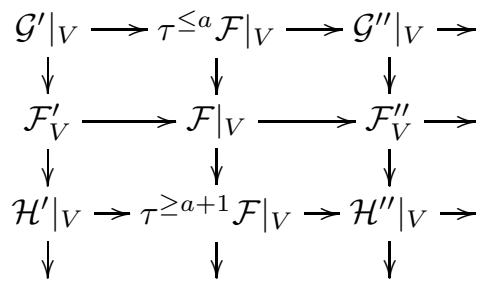

Recall that all rows and columns in this diagram are distinguished triangles. The categories $\mathcal{D}_{G}^{\mathrm{b}}(V)^{[a, b]}$ and $\mathcal{D}_{G}^{\mathrm{b}}(V)^{\leq n}$ are stable under extensions, so we clearly have $\mathcal{F}_{V}^{\prime} \in \mathcal{D}_{G}^{\mathrm{b}}(V)^{\leq n} \cap \mathcal{D}_{G}^{\mathrm{b}}(V)^{[a, b]}$. It is also evident that $\mathcal{F}_{V}^{\prime \prime} \in \mathcal{D}_{G}^{\text {supp }}(V, V \cap Z)$. Since $\mathcal{D}_{G}^{\mathrm{b}}(V)^{\leq d-n-1}$ is stable under extensions, we have $\mathbb{D}\left(\mathcal{F}_{V}^{\prime \prime}\right) \in \mathcal{D}_{G}^{\mathrm{b}}(V)^{\leq d-n-1}$ as well. Now, by Lemma 2.3. we can find a distinguished triangle

$$
\mathcal{F}^{\prime} \rightarrow \mathcal{F} \rightarrow \mathcal{F}^{\prime \prime} \rightarrow
$$

with $\mathcal{F}^{\prime} \in \mathcal{D}_{G}^{\mathrm{b}}(X)^{[a, b]},\left.\mathcal{F}^{\prime}\right|_{V} \simeq \mathcal{F}_{V}^{\prime}$, and $\left.\mathcal{F}^{\prime \prime}\right|_{V} \simeq \mathcal{F}_{V}^{\prime \prime}$, and such that the restriction to $V$ of this distinguished triangle is isomorphic to the middle row of the diagram obtained from the 9-lemma above. This distinguished triangle has all the properties we seek. 
Below, we will make use of the "*" operation on a triangulated category introduced in [BBD, §1.3]. Recall that if $\mathcal{A}_{1}$ and $\mathcal{A}_{2}$ are classes of objects in a triangulated category, then

$$
\mathcal{A}_{1} * \mathcal{A}_{2}
$$

is the class of all objects $A$ that can be embedded in a distinguished triangle

$$
A_{1} \rightarrow A \rightarrow A_{2} \rightarrow
$$

with $A_{1} \in \mathcal{A}_{1}$ and $A_{2} \in \mathcal{A}_{2}$. According to [BBD, Lemme 1.3.10], the $*$ operation is associative, so expressions like $\mathcal{A}_{1} * \mathcal{A}_{2} * \mathcal{A}_{3}$ are well-defined. This fact will be used freely below.

Lemma 8.3. Let $U \subset X$ be an open subscheme, and let $Z \subset X$ be the complementary closed subscheme. Let $\mathcal{F} \in \mathcal{D}_{G}^{\mathrm{b}}(X)^{[a, b]}$ be such that $\left.\mathcal{F}\right|_{U} \in \mathcal{D}_{G}^{\mathrm{b}}(U)_{\diamond}^{\leq n}$. Then

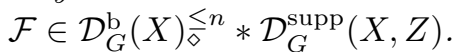

Proof. This lemma follows by noetherian induction from Lemma 8.2. Specifically, let us assume that the lemma is known if $Z$ is replaced by any proper closed subscheme. (In the base case, where $U=X$ and $Z=\varnothing$, the lemma is trivial.) Let $\mathcal{F} \in \mathcal{D}_{G}^{\mathrm{b}}(X)^{[a, b]}$ with $\left.\mathcal{F}\right|_{U} \in \mathcal{D}_{G}^{\mathrm{b}}(U)_{\diamond}^{\leq n}$, and choose a generic point $x \in Z$. Lemma 8.2 tells us that

$$
\mathcal{F} \in\left\{\mathcal{F}^{\prime}\right\} * \mathcal{D}_{G}^{\text {supp }}(X, Z)
$$

for some $\mathcal{F}^{\prime} \in \mathcal{D}_{G}^{\mathrm{b}}(X)^{[a, b]}$ with $\left.\mathcal{F}^{\prime}\right|_{V} \in \mathcal{D}_{G}^{\mathrm{b}}(V)^{\leq n}$, where $V \supset U$. Let $Y \subset Z$ be a closed subscheme structure on the complement of $V \cap Z$. Then $Y$ is a proper closed subscheme of $Z$, so we know by assumption that

$$
\mathcal{F}^{\prime} \in \mathcal{D}_{G}^{\mathrm{b}}(X)_{\diamond}^{\leq n} * \mathcal{D}_{G}^{\mathrm{supp}}(X, Z) .
$$

It is clear that $\mathcal{D}_{G}^{\text {supp }}(X, Z) * \mathcal{D}_{G}^{\text {supp }}(X, Z)=\mathcal{D}_{G}^{\text {supp }}(X, Z)$, and since $*$ is associative, the lemma follows.

Proposition 8.4. We have $\mathcal{D}_{G}^{\mathrm{b}}(X)={ }^{p} \mathcal{D}_{G}^{\mathrm{b}}(X)^{\leq 0} *^{p} \mathcal{D}_{G}^{\mathrm{b}}(X)^{\geq 1}$.

Proof. Let $\mathcal{F} \in \mathcal{D}_{G}^{\mathrm{b}}(X)$. We proceed by noetherian induction on the support of $\mathcal{F}$. Suppose $\mathcal{F}$ is supported on a closed subscheme $\kappa: Y \rightarrow X$. Then there is some $\mathcal{F}_{1} \in \mathcal{D}_{G}^{\mathrm{b}}(Y)$ such that $\mathcal{F} \simeq \kappa_{*} \mathcal{F}_{1}$. Choose a generic point $x \in Y^{\text {gen }}$ such that $p(x) \leq p(y)$ for all $y \in Y^{\text {gen }}$. (This minimum value of $p$ must be achieved on one of the finitely many generic points of $Y$.) Now, apply Lemma 8.2 to $\mathcal{F}_{1}$. (In the notation of that statement, we are taking $U=\varnothing$.) We obtain an open subscheme $V \subset Y$ containing $x$, and a distinguished triangle

$$
\mathcal{F}_{1}^{\prime} \rightarrow \mathcal{F}_{1} \rightarrow \mathcal{F}_{1}^{\prime \prime} \rightarrow
$$

where $\left.\mathcal{F}_{1}^{\prime}\right|_{V} \in \mathcal{D}_{G}^{\mathrm{b}}(V)_{\diamond}^{\leq p(x)}$ and $\left.\mathbb{D}\left(\mathcal{F}_{1}^{\prime \prime}\right)\right|_{V} \in \mathcal{D}_{G}^{\mathrm{b}}(V)_{\diamond}^{\leq \operatorname{scod} \overline{G x}-p(x)-1}=\mathcal{D}_{G}^{\mathrm{b}}(V)_{\diamond}^{\leq \bar{p}(x)-1}$. Next, let $Z \subset Y$ be a closed subscheme complementary to $V$. By Lemma 8.3 we know that

$\mathcal{F}_{1}^{\prime} \in \mathcal{D}_{G}^{\mathrm{b}}(Y)_{\diamond}^{\leq p(x)} * \mathcal{D}_{G}^{\mathrm{supp}}(Y, Z) \quad$ and $\quad \mathbb{D}\left(\mathcal{F}_{1}^{\prime \prime}\right) \in \mathcal{D}_{G}^{\mathrm{b}}(Y)_{\diamond}^{\leq \bar{p}(x)-1} * \mathcal{D}_{G}^{\mathrm{supp}}(Y, Z)$.

From the distinguished triangle (8.2), we see now that

$$
\begin{aligned}
\mathcal{F}_{1} \in & \left(\mathcal{D}_{G}^{\mathrm{b}}(Y)_{\diamond}^{\leq p(x)} * \mathcal{D}_{G}^{\mathrm{supp}}(Y, Z)\right) * \mathbb{D}\left(\mathcal{D}_{G}^{\mathrm{b}}(Y)_{\diamond}^{\leq \bar{p}(x)-1} * \mathcal{D}_{G}^{\mathrm{supp}}(Y, Z)\right) \\
& =\mathcal{D}_{G}^{\mathrm{b}}(Y)_{\diamond}^{\leq p(x)} * \mathcal{D}_{G}^{\mathrm{supp}}(Y, Z) * \mathbb{D}\left(\mathcal{D}_{G}^{\mathrm{supp}}(Y, Z)\right) * \mathbb{D}\left(\mathcal{D}_{G}^{\mathrm{b}}(Y)_{\diamond}^{\leq \bar{p}(x)-1}\right) \\
& =\mathcal{D}_{G}^{\mathrm{b}}(Y)^{\leq p p(x)} * \mathcal{D}_{G}^{\mathrm{supp}}(Y, Z) * \mathbb{D}\left(\mathcal{D}_{G}^{\mathrm{b}}(Y)^{\leq \bar{p}(x)-1}\right) .
\end{aligned}
$$


Recall from Lemma 7.6 that $\kappa_{*}$ takes $\mathcal{D}_{G}^{\mathrm{b}}(Y)_{\diamond}^{\leq n}$ to $\mathcal{D}_{G}^{\mathrm{b}}(X)_{\diamond}^{\leq n}$. This functor also commutes with $\mathbb{D}$, so we find that

$\mathcal{F} \in\left(\mathcal{D}_{G}^{\text {supp }}(X, Y) \cap \mathcal{D}_{G}^{\mathrm{b}}(X)_{\diamond}^{\leq p(x)}\right) * \mathcal{D}_{G}^{\text {supp }}(X, Z) * \mathbb{D}\left(\mathcal{D}_{G}^{\text {supp }}(X, Y) \cap \mathcal{D}_{G}^{\mathrm{b}}(X)_{\diamond}^{\leq \bar{p}(x)-1}\right)$.

Now, since $p(x)$ is the minimum value of $p$ on $Y^{\text {gen }}$, Proposition 8.1 allows us to deduce that

$$
\begin{aligned}
\mathcal{F} \in{ }^{p} \mathcal{D}_{G}^{\mathrm{b}}(X)^{\leq 0} * \mathcal{D}_{G}^{\mathrm{supp}}(X, Z) * \mathbb{D}\left({ }^{\bar{p}} \mathcal{D}_{G}^{\mathrm{b}}(X)^{\leq-1}\right) & \\
& ={ }^{p} \mathcal{D}_{G}^{\mathrm{b}}(X)^{\leq 0} * \mathcal{D}_{G}^{\text {supp }}(X, Z) *{ }^{p} \mathcal{D}_{G}^{\mathrm{b}}(X)^{\geq 1} .
\end{aligned}
$$

We have assumed inductively that $\mathcal{D}_{G}^{\text {supp }}(X, Z) \subset{ }^{p} \mathcal{D}_{G}^{\mathrm{b}}(X) \leq 0{ }^{p}{ }^{p} \mathcal{D}_{G}^{\mathrm{b}}(X) \geq 1$, so we can conclude that $\mathcal{F} \in{ }^{p} \mathcal{D}_{G}^{\mathrm{b}}(X)^{\leq 0}{ }^{p}{ }^{p} \mathcal{D}_{G}^{\mathrm{b}}(X)^{\geq 1}$.

Proof of Theorem 7.4. It is obvious from the definitions that

$$
{ }^{p} \mathcal{D}_{G}^{\mathrm{b}}(X)^{\leq-1} \subset{ }^{p} \mathcal{D}_{G}^{\mathrm{b}}(X)^{\leq 0} \quad \text { and } \quad{ }^{p} \mathcal{D}_{G}^{\mathrm{b}}(X)^{\geq 0} \subset{ }^{p} \mathcal{D}_{G}^{\mathrm{b}}(X)^{\geq 1} .
$$

The other axioms for a $t$-structure have been checked in Propositions 7.9 and 8.4 Thus, the categories $\left({ }^{p} \mathcal{D}_{G}^{\mathrm{b}}(X) \leq 0,{ }^{p} \mathcal{D}_{G}^{\mathrm{b}}(X)^{\geq 0}\right)$ define a $t$-structure on $\mathcal{D}_{G}^{\mathrm{b}}(X)$.

Next, we show that it is nondegenerate. We first prove that there is no nonzero object belonging to ${ }^{p} \mathcal{D}_{G}^{-}(X)^{\leq n}$ for all $n \in \mathbb{Z}$. We proceed by noetherian induction: assume that for any proper closed subscheme $Z \subset X$, we have $\bigcap_{n \in \mathbb{Z}}{ }^{p} \mathcal{D}_{G}^{-}(Z)^{\leq n}=$ $\{0\}$. The base case is that in which $X$ contains no nonempty proper closed (Ginvariant) subschemes. In that case, we simply have ${ }^{p} \mathcal{D}_{G}^{-}(X)^{\leq 0}=\mathcal{D}_{G}^{-}(X)_{\mathbf{\Delta}}^{\leq p(x)}=$ $\mathcal{D}_{G}^{-}(X)_{\diamond}^{\leq p(x)}$, where $x$ is any generic point of $X$. Now, if we had an object $\mathcal{F}$ that was in ${ }^{p} \mathcal{D}_{G}^{-}(X)^{\leq n}$ for all $n \in \mathbb{Z}$, that would imply that for every $k \in \mathbb{Z}$, $H^{k}(\mathcal{F}) \in \mathcal{C}_{G}(X)_{\leq p(x)+n-k}$ for all $n \in \mathbb{Z}$. Using axiom (S5), we see that that implies that $H^{k}(\mathcal{F})=0$ for all $k$, so in fact $\mathcal{F}=0$, as desired.

In the general case, let $\mathcal{F} \in \mathcal{D}_{G}^{-}(X)$ be a nonzero object. Then there certainly exists some proper closed subscheme $i: Z \hookrightarrow X$ such that $L i^{*} \mathcal{F} \in \mathcal{D}_{G}^{-}(Z)$ is nonzero. If $\mathcal{F} \in{ }^{p} \mathcal{D}_{G}^{-}(X)^{\leq n}$ for all $n$, then by Lemma 7.6. $L i^{*} \mathcal{F} \in{ }^{p} \mathcal{D}_{G}^{-}(Z)^{\leq n}$ for all $n$, but that is a contradiction. We conclude that $\bigcap_{n \in \mathbb{Z}}{ }^{p} \mathcal{D}_{G}^{-}(X) \leq n=\{0\}$.

Applying $\mathbb{D}$ lets us conclude that there is no nonzero object belonging to all ${ }^{p} \mathcal{D}_{G}^{+}(X)^{\geq n}$ either. Thus, the $t$-structure on $X$ is nondegenerate.

Finally, we show that it is bounded. Any $\mathcal{F} \in \mathcal{D}_{G}^{\mathrm{b}}(X)$ has finitely many nonzero cohomology sheaves, of course. For each $k \in \mathbb{Z}$ such that $H^{k}(\mathcal{F}) \neq 0$, let $v_{k} \in \mathbb{Z}$ be such that $H^{k}(\mathcal{F}) \in \mathcal{C}_{G}(X)_{\leq v_{k}}$. (Such a $v_{k}$ exists by axiom (S5 5 .) Then, let $d$ be the minimal value of the perversity $p$, and define

$$
n=\max \left\{v_{k}+k \mid H^{k}(\mathcal{F}) \neq 0\right\}-d .
$$

We then have $v_{k} \leq n+d-k$ for each $k$, so we see that $H^{k}(\mathcal{F}) \in \mathcal{C}_{G}(X)_{\leq n+d-k}$.

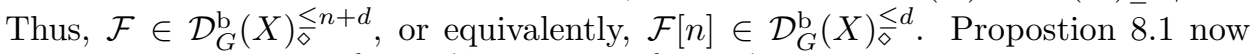
tells us that $\mathcal{F}[n] \in{ }^{P} \mathcal{D}_{G}^{\mathrm{b}}(X) \leq 0$, or $\mathcal{F} \in{ }^{P} \mathcal{D}_{G}^{\mathrm{b}}(X) \leq n$. A similar argument allows to find an integer $m$ such that $\mathbb{D}(\mathcal{F}) \in \bar{p}_{G}^{\mathrm{b}}(X)^{\leq m}$, and from that we deduce that $\mathcal{F} \in{ }^{p} \mathcal{D}_{G}^{\mathrm{b}}(X)^{\geq-m}$. Thus, the staggered $t$-structure is bounded.

Corollary 8.5. Let $i: Z \hookrightarrow X$ be a closed subscheme. Then $i_{*}$ takes ${ }^{p} \mathcal{D}_{G}^{\mathrm{b}}(Z)^{\leq 0}$ to ${ }^{p} \mathcal{D}_{G}^{\mathrm{b}}(X) \leq 0$ and ${ }^{p} \mathcal{D}_{G}^{\mathrm{b}}(Z)^{\geq 0}$ to ${ }^{p} \mathcal{D}_{G}^{\mathrm{b}}(X)^{\geq 0}$. 
Proof. . Let $\mathcal{F} \in{ }^{p} \mathcal{D}_{G}^{\mathrm{b}}(Z) \leq 0$. Since we now know that $\left({ }^{p} \mathcal{D}_{G}^{\mathrm{b}}(X) \leq 0,{ }^{p} \mathcal{D}_{G}^{\mathrm{b}}(X)^{\geq 0}\right)$ is a $t$ structure on $\mathcal{D}_{G}^{\mathrm{b}}(X)$, we know that $i_{*} \mathcal{F} \in{ }^{p} \mathcal{D}_{G}^{\mathrm{b}}(X) \leq 0$ if and only if $\operatorname{Hom}\left(i_{*} \mathcal{F}, \mathcal{G}\right)=0$ for all $\mathcal{G} \in{ }^{p} \mathcal{D}_{G}^{\mathrm{b}}(X)^{\geq 1}$. $\operatorname{But} \operatorname{Hom}\left(i_{*} \mathcal{F}, \mathcal{G}\right) \simeq \operatorname{Hom}\left(\mathcal{F}, R i^{!} \mathcal{G}\right)$, and $R i^{!} \mathcal{G} \in \mathcal{D}_{G}^{\mathrm{b}}(Z)^{\geq 1}$ by Lemma 7.6. We see that $\operatorname{Hom}\left(\mathcal{F}, R i^{!} \mathcal{G}\right)=0$ for all $\mathcal{G} \in \mathcal{D}_{G}^{\mathrm{b}}(X)^{\geq 1}$, so $i_{*} \mathcal{F} \in$ $\mathcal{D}_{G}^{\mathrm{b}}(X)^{\leq 0}$. A similar argument shows that $i_{*}$ takes ${ }^{p} \mathcal{D}_{G}^{\mathrm{b}}(Z)^{\geq 0}$ to ${ }^{p} \mathcal{D}_{G}^{\mathrm{b}}(X)^{\geq 0}$.

\section{Simple Staggered Sheaves}

Recall from Section 1 that under certain circumstances, simple objects in the category of perverse coherent sheaves have a remarkably easy description (in terms of "middle-extension functors"), and that all objects in the category have finite length. In this section, we develop analogues of these results for staggered sheaves. The proofs follow those given in [B1, §3.2] for perverse coherent sheaves quite closely, and in many cases we have omitted most details.

In this section and the following one, we assume that the base scheme over which we are working is Spec $\mathbb{k}$ for some field $\mathbb{k}$, and we assume that $G$ is a linear algebraic group over $\mathbb{k}$. An orbit in $X$ will mean a reduced, locally closed subscheme isomorphic to a homogeneous space for $G$.

Let $j: U \hookrightarrow X$ be a dense open subscheme, and let $Z \subset X$ be its complement. Given a perversity $p$, define new functions $p^{-}, p^{+}: X^{\text {gen }} \rightarrow \mathbb{Z}$ by

$$
p^{-}(x)=\left\{\begin{array}{ll}
p(x) & \text { if } x \in U^{\text {gen }}, \\
p(x)-1 & \text { if } x \notin U^{\text {gen }},
\end{array} \quad p^{+}(x)= \begin{cases}p(x) & \text { if } x \in U^{\text {gen }} \\
p(x)+1 & \text { if } x \notin U^{\text {gen }}\end{cases}\right.
$$

These are not necessarily perversities: they need not, in general, have the monotonicity and comonotonicity properties of Definition 7.2 so they may not give rise to $t$-structures on $\mathcal{D}_{G}^{\mathrm{b}}(X)$. Nevertheless, the definitions of the categories ${ }^{-} \mathcal{D}_{G}^{-}(X) \leq 0$ and $p^{+} \mathcal{D}_{G}^{+}(X)^{\geq 0}$ still make sense.

Lemma 9.1. Let $\mathcal{F} \in{ }^{p} \mathcal{M}(X)$. Then:

(1) $\mathcal{F} \in p^{-} \mathcal{D}_{G}^{\mathrm{b}}(X) \leq 0$ if and only if $\operatorname{Hom}(\mathcal{F}, \mathcal{G})=0$ for all $\mathcal{G} \in{ }^{p} \mathcal{M}(X) \cap$ $\mathcal{D}_{G}^{\text {supp }}(X, Z)$.

(2) $\mathcal{F} \in p^{+} \mathcal{D}_{G}^{\mathrm{b}}(X)^{\geq 0}$ if and only if $\operatorname{Hom}(\mathcal{G}, \mathcal{F})=0$ for all $\mathcal{G} \in{ }^{p} \mathcal{M}(X) \cap$ $\mathcal{D}_{G}^{\text {supp }}(X, Z)$.

Proof. This follows from Lemma 7.6. Corollary 8.5, and general properties of $t$ structures, as in the proof of [B1, Lemma 6].

Proposition 9.2. Assume that $p^{-}$and $p^{+}$are themselves perversities, and define a full subcategory ${ }^{p} \mathcal{M}^{! *}(X) \subset{ }^{p} \mathcal{M}(X)$ by ${ }^{p} \mathcal{M}^{! *}(X)=p^{-} \mathcal{D}_{G}^{\mathrm{b}}(X) \leq 0 \cap{ }^{p^{+}} \mathcal{D}_{G}^{\mathrm{b}}(X) \geq 0$. The functor $j^{*}$ induces an equivalence of categories ${ }^{p} \mathcal{M}^{! *}(X) \rightarrow{ }^{p} \mathcal{M}(U)$.

Proof. The arguments given in either [B1, Theorem 2] or [AS1, Proposition 2.3] can be repeated verbatim, with one minor change: references to [B1, Lemma 6] should be replaced by references to Lemma 9.1 above. We omit further details.

Remark 9.3. The assumption that $p^{-}$and $p^{+}$are themselves perversities has as its main consequence the requirement that for any $x \in U^{\text {gen }}$ and $y \in Z^{\text {gen }}$ with $y \in \overline{G x}$, we must have $\operatorname{scod} \overline{G y} \geq \operatorname{scod} \overline{G x}+2$. (This follows easily from the monotonicity and comonotonicity requirements and the fact that $p^{+}(y)=p^{-}(y)+2$.) A similar restriction appears in [B1, §3.2]. 
A different approach, introduced in AS1, Propostions 2.3, 2.6], allows one to prove a version of this result in which it is not necessary to assume that $p^{-}$and $p^{+}$are perversities. (To be more precise, their definitions are changed in such a manner that they are guaranteed to be perversities.) That approach could certainly be duplicated here.

However, the resulting equivalence of categories has the disadvantage that it relates ${ }^{p} \mathcal{M}^{! *}(X)$ to a certain full (but not Serre!) subcategory of ${ }^{p} \mathcal{M}(U)$, not ${ }^{p} \mathcal{M}(U)$ itself. We will not require the generalized form of the middle-extension functor, and indeed, the generalized version cannot be used to prove an analogue of Theorem 9.9. We will therefore content ourselves with the version stated above.

Definition 9.4. The inverse equivalence to the one described in Proposition 9.2 denoted $j_{!_{*}}:{ }^{p} \mathcal{M}(U) \rightarrow{ }^{p} \mathcal{M}^{! *}(X)$, is called the middle-extension functor.

Definition 9.5. Let $\kappa: Y \hookrightarrow X$ be a closed subscheme, and let $h: V \hookrightarrow Y$ be an open subscheme of $Y$. If $h_{!_{*}}$ is defined, then given any $\mathcal{F} \in{ }^{p} \mathcal{M}(V)$, we define an object of ${ }^{p} \mathcal{M}(X)$ by

$$
\mathcal{I C}(Y, \mathcal{F})=\kappa_{*}\left(h_{! *} \mathcal{F}\right) .
$$

This is called the (staggered) intersection cohomology complex associated to $\mathcal{F}$.

Proposition 9.6. Suppose $X$ has the property that its associated reduced scheme $X_{\text {red }}$ is a single $G$-orbit. Let $d$ be the value of the perversity $p$ at any generic point of $X$. Then

$$
{ }^{p} \mathcal{M}(X)=\left\{\mathcal{F} \in \mathcal{D}_{G}^{\mathrm{b}}(X) \mid \mathcal{F} \text { is concentrated in staggered degree } d\right\} .
$$

In addition, let $t: X_{\text {red }} \rightarrow X$ be the inclusion map. For any irreducible vector bundle $\mathcal{L} \in \mathcal{C}_{G}\left(X_{\mathrm{red}}\right), t_{*} \mathcal{L}[-d+\operatorname{step} \mathcal{L}]$ is a simple object of ${ }^{p} \mathcal{M}(X)$, and every simple object arises in this way.

(See Remark 7.3 for the notion of staggered degree.)

Proof. Since $X_{\text {red }}$ is a single $G$-orbit, $X$ obviously has no nonempty proper open subschemes, and no nonempty proper closed subschemes except those whose underlying topological space is all of $X$. We see, therefore, that $\mathcal{D}_{G}^{-}(X)_{\Delta}^{\leq n}=\mathcal{D}_{G}^{-}(X)_{\diamond}^{\leq n}$. It follows then from Proposition 8.1 that ${ }^{p} \mathcal{D}_{G}^{-}(X)^{\leq 0}=\mathcal{D}_{G}^{-}(X)_{\stackrel{\Delta}{\circ}}^{\leq}$. Now, suppose $\mathcal{F} \in{ }^{p} \mathcal{D}_{G}^{+}(X)^{\geq 0}$, so that $\mathbb{D}(\mathcal{F}) \in \bar{p}^{\bar{p}} \bar{G}_{G}^{-}(X)^{\leq 0}=\mathcal{D}_{G}^{-}(X)_{\grave{s}}^{\leq \operatorname{scod} X-d}$. A straightforward induction argument on the number of nonzero cohomology sheaves of $\mathcal{F}$, combined with Remark 3.2 and Lemma 6.6, shows that $H^{k}(\mathcal{F}) \in \mathcal{C}_{G}(X)_{\geq d-k}$ for all $k$. We conclude, therefore, that $\mathcal{F} \in{ }^{p} \mathcal{M}(X)$ if and only if $H^{k}(\mathcal{F}) \in$ $\mathcal{C}_{G}(X)_{\leq d-k} \cap \mathcal{C}_{G}(X)_{\geq d-k}$, as desired.

Under the circumstances of this proposition, we see that ${ }^{p} \mathcal{M}(X)$ is stable under all (standard) truncation functors. (This is certainly not true in general.) It is then obvious that a simple object of ${ }^{p} \mathcal{M}(X)$ must be concentrated in a single degree. Determining the simple objects in ${ }^{p} \mathcal{M}(X)$ then essentially reduces to determining the simple objects in $\mathcal{C}_{G}(X)$ (up to shifting, to give objects the correct staggered degree). Of course, every simple object of $\mathcal{C}_{G}(X)$ is supported on $X_{\text {red }}$. Since $X_{\text {red }}$ is a single $G$-orbit, all objects of $\mathcal{C}_{G}\left(X_{\text {red }}\right)$ are locally free; i.e., they are vector bundles on $X_{\text {red }}$. Thus, the simple objects of ${ }^{p} \mathcal{M}(X)$ are precisely the objects in $\mathcal{D}_{G}^{\mathrm{b}}(X)$ of the form $t_{*} \mathcal{L}[-d+$ step $\mathcal{L}]$, with $\mathcal{L} \in \mathcal{C}_{G}\left(X_{\text {red }}\right)$ an irreducible vector bundle. 
If $C \subset X$ is a single $G$-orbit, we write $p(C)$ for the value of the perversity function $p$ at any generic point of $C$. They are necessarily all equal, so $p(C)$ is well-defined.

Theorem 9.7. Let $C \subset X$ be a $G$-orbit. Assume that for any point $y \in \bar{C}^{\text {gen }} \backslash C^{\text {gen }}$, we have $p(y)>p(C)$ and $\bar{p}(y)>\bar{p}(C)$. Then, for any object $\mathcal{F} \in{ }^{p} \mathcal{M}(X) \cap$ $\mathcal{D}_{G}^{\text {supp }}(X, \bar{C})$, the following conditions are equivalent:

(1) $\mathcal{F}$ is a simple staggered sheaf.

(2) $\mathcal{F} \simeq \mathcal{I C}(\bar{C}, \mathcal{L}[-p(C)+$ step $\mathcal{L}])$, where $\mathcal{L} \in \mathcal{C}_{G}(C)$ is some irreducible vector bundle.

Remark 9.8. The analogue of this statement for perverse coherent sheaves, given in [B1, Corollary 4], is significantly stronger: the conditions on the perversity are not imposed at the outset, but deduced from the assumption that $\mathcal{F}$ is simple, using Nakayama's Lemma; furthermore, the fact that the support of any simple object is the closure a single $G$-orbit is obtained from Rosenlicht's Theorem. This stronger statement is not true in general for staggered sheaves, in part because there is no analogue of Nakayama's Lemma for $s$-structures: for a sheaf $\mathcal{F} \in \mathcal{C}_{G}(X)$, knowing that $\left.\mathcal{F}\right|_{U} \notin \mathcal{C}_{G}(U)_{\leq w}$ for some $w$ and some open subscheme $U$ imposes no restrictions at all on the behavior of $\mathcal{F}$ with respect to the $s$-structure on the complementary closed subscheme $i: Z \hookrightarrow X$. Similarly, for $\mathcal{F} \in{ }^{p} \mathcal{M}(X)$, knowing that $\left.\mathcal{F}\right|_{U} \notin \mathcal{D}_{G}^{-}(U)_{\diamond}^{\leq n}$ for some $n$ does not imply that $L i^{*} \mathcal{F} \notin \mathcal{D}_{G}^{-}(Z)_{\diamond}^{\leq n}$. Moreover, we cannot invoke Rosenlicht's Theorem, because the perversity only gives us information about the staggered codimensions of closed subschemes, not their actual codimensions.

Proof. We reserve the notation $\bar{C}$ for the reduced closed subscheme structure on the closure of $C$. Let $\mathcal{F} \in \mathcal{D}_{G}^{\text {supp }}(X, \bar{C})$ be a simple object of ${ }^{p} \mathcal{M}(X)$. Let $\kappa: Y \hookrightarrow X$ be a closed subscheme structure on the underlying topological space of $\bar{C}$ such that $\mathcal{F}$ is supported on $Y$; suppose $\mathcal{F} \simeq \kappa_{*} \mathcal{F}_{1}$. Let $h: V \hookrightarrow Y$ be the open subscheme with the same underlying topological space as $C$. It is clear from the hypotheses on the perversity that the middle-extension functor $h_{! *}$ is defined, and then from the construction of $h_{! *}$ in the proof of Proposition 9.2, we see that $h_{! *}\left(\left.\mathcal{F}_{1}\right|_{V}\right)$ is a subquotient of $\mathcal{F}_{1}$. Since the latter is simple, we see that $\mathcal{F}_{1} \simeq h_{l_{*}}\left(\left.\mathcal{F}_{1}\right|_{V}\right)$, and hence that $\mathcal{F} \simeq \mathcal{I C}\left(Y,\left.\mathcal{F}_{1}\right|_{V}\right)$. Now, because $h_{\text {!* }}$ gives an equivalence of categories between ${ }^{p} \mathcal{M}(V)$ and ${ }^{p} \mathcal{M}^{! *}(Y)$, we know that $\left.\mathcal{F}_{1}\right|_{V}$ must be a simple object of ${ }^{p} \mathcal{M}(V)$, and then by Proposition 9.6, it is a shift of an irreducible vector bundle on $C$. Thus, $\mathcal{F}$ must be of the form $\mathcal{I C}(\bar{C}, \mathcal{L}[-p(C)+\operatorname{step} \mathcal{L}])$, where $\mathcal{L} \in \mathcal{C}_{G}(C)$ is an irreducible vector bundle.

Conversely, if $\mathcal{L}$ is an irreducible vector bundle on $C$, then it follows from Lemma 9.1 that $\mathcal{I C}(\bar{C}, \mathcal{L}[-p(C)+\operatorname{step} \mathcal{L}])$ is a simple object in ${ }^{p} \mathcal{M}(X)$.

Theorem 9.9. Suppose $G$ acts on $X$ with finitely many orbits. In addition, assume that for any two orbits $C, C^{\prime}$ with $C^{\prime} \subset \bar{C}$ and $C^{\prime} \neq C$, we have $p\left(C^{\prime}\right)>p(C)$ and $\bar{p}\left(C^{\prime}\right)>\bar{p}(C)$. Then ${ }^{p} \mathcal{M}(X)$ is a finite-length category.

Proof. Identical to [B1, Corollary 5].

A perversity satisfying the conditions of this theorem can be found if $\operatorname{scod} C^{\prime} \geq$ scod $C+2$ whenever $C^{\prime} \subset \bar{C}, C^{\prime} \neq C$. In particular, if every orbit closure $\bar{C}$ has even staggered codimension in $X$, then the perversity defined by

$$
p(C)=\frac{1}{2} \operatorname{scod} C,
$$


called the middle perversity, gives rise to a self-dual, finite-length category ${ }^{p} \mathcal{M}(X)$.

\section{Schemes with Finitely Many G-ORBits}

In order to study examples of staggered sheaves, one must of course be able to produce explicit $s$-structures. Most of the axioms are quite easy to check, but axiom (S9) appears quite intimidating at first glance. Fortunately, as we will see in this section, if $G$ acts on $X$ with finitely many orbits, it is not necessary to check this axiom. (We retain the assumption from Section 9 that $X$ is a scheme of finite type over a field $\mathbb{k}$, and that $G$ is a linear algebraic group over $\mathbb{k}$.)

We first prove a criterion for adhesiveness in a particularly easy case.

Proposition 10.1. Let $Z \subset X$ be a reduced closed subscheme with an almost sstructure, and assume that axiom (A1) of Definition 5.1 holds. If $Z$ is a union of finitely many closed $G$-orbits, then $Z$ is adhesive, and its almost s-structure is automatically an s-structure.

Proof. Because $Z$ is a union of finitely many closed $G$-orbits, it has no nonempty proper dense open $\left(G\right.$-invariant) subschemes. It follows that $\tilde{\mathcal{C}}_{G}(Z)_{\geq w}=\mathcal{C}_{G}(Z)_{\geq w}$ for each $w$, and hence that $\mathcal{C}_{G}(Z)_{\geq w}$ is closed under quotients. The same applies to any other subscheme structure on $\underline{Z}$, so we conclude that $\mathcal{C}_{G}^{\text {supp }}(X, Z)_{\geq w}$ is closed under quotients, and is therefore a Serre subcategory of $\mathcal{C}_{G}(X)$.

Objects in the category $\mathcal{Q}_{G}(X)$ of $G$-equivariant quasicoherent sheaves have injective hulls, by [H, §II.7]. Moreover, the injective hull of any sheaf supported on $\underline{Z}$ must itself be supported on $\underline{Z}$. Consider the category

$$
\mathcal{Q}_{G}^{\text {supp }}(X, Z)_{\geq w}=\left\{\mathcal{F} \in \mathcal{Q}_{G}(X) \mid \begin{array}{c}
\text { every coherent subsheaf } \\
\text { of } \mathcal{F} \text { is in } \mathcal{C}_{G}^{\text {supp }}(X, Z)_{\geq w}
\end{array}\right\} .
$$

Note that all sheaves in $\mathcal{Q}_{G}^{\text {supp }}(X, Z)_{\geq w}$ are supported on $\underline{Z}$.

We show now that $\mathcal{Q}_{G}^{\text {supp }}(X, Z)_{\geq w}$ is stable under quotients. Given a sheaf $\mathcal{F} \in \mathcal{Q}_{G}^{\text {supp }}(X, Z)_{\geq w}$, let $\mathcal{G}$ be a quotient of $\mathcal{F}$. Every coherent subsheaf of $\mathcal{G}$ is a quotient of some coherent subsheaf of $\mathcal{F}$. The latter is in $\mathcal{C}_{G}^{\text {supp }}(X, Z)_{\geq w}$, and since that category is closed under quotients, we see that $\mathcal{G} \in \mathcal{Q}_{G}^{\mathrm{supp}}(X, Z)_{\geq w}$.

Next, given $\mathcal{F} \in \mathcal{Q}_{G}^{\text {supp }}(X, Z)_{\geq w}$, let $\mathcal{I}$ be its injective hull. We claim that $\mathcal{I} \in$ $\mathcal{Q}_{G}^{\text {supp }}(X, Z)_{\geq w}$. Indeed, suppose $\mathcal{I}$ had coherent subsheaf $\mathcal{H}$ not in $\mathcal{C}_{G}^{\text {supp }}(X, Z)_{\geq w}$. $\mathcal{H}$ is supported on some subscheme structure $i^{\prime}: Z^{\prime} \hookrightarrow X$ on $\underline{Z}$. Say $\mathcal{H} \simeq i_{*}^{\prime} \mathcal{H}_{1}$. Then $\mathcal{H}_{1} \notin \mathcal{C}_{G}\left(Z^{\prime}\right)_{\geq w}$, so the sheaf $\sigma_{\leq w-1} \mathcal{H}_{1} \in \mathcal{C}_{G}\left(Z^{\prime}\right)_{\leq w-1}$ must be nonzero. Consider the sheaf $\mathcal{H}_{2}=i_{*}^{\prime}\left(\sigma_{\leq w-1} \mathcal{H}_{1}\right)$. We may regard $\mathcal{H}_{2}$ as a subsheaf of $\mathcal{I}$. Because $\mathcal{I}$ is an injective hull of $\mathcal{F}$, we know that $\mathcal{H}_{2} \cap \mathcal{F}$ is nonzero. But then $\mathcal{H}_{2} \cap \mathcal{F}$ is a coherent subsheaf of $\mathcal{F}$ that is not in $\mathcal{C}_{G}^{\text {supp }}(X, Z)_{\geq w}$, contradicting the assumption that $\mathcal{F} \in \mathcal{Q}_{G}^{\text {supp }}(X, Z)_{\geq w}$.

From the two preceding paragraphs, we see that every sheaf in $\mathcal{Q}_{G}^{\text {supp }}(X, Z)_{\geq w}$ admits an injective resolution all of whose terms are in $\mathcal{Q}_{G}^{\text {supp }}(X, Z)_{\geq w}$. In particular, given a coherent sheaf $\mathcal{G} \in \mathcal{C}_{G}^{\text {supp }}(X, Z)_{\geq w}$, we see that $\mathcal{G}$ has an injective resolution by sheaves in $\mathcal{Q}_{G}^{\text {supp }}(X, Z)_{\geq w}$. Now, suppose $\mathcal{F} \in \mathcal{C}_{G}^{\text {loc }}(X, Z)_{\leq w-1}$. Given a sheaf $\mathcal{I} \in \mathcal{Q}_{G}^{\text {supp }}(X, Z)_{\geq w}$, we see that $\operatorname{Hom}(\mathcal{F}, \mathcal{I})=0$ (because the image of any nonzero morphism $\overline{\mathcal{F}} \rightarrow \mathcal{I}$ would be a coherent subsheaf of $\mathcal{I}$ not in $\left.\mathcal{C}_{G}^{\text {supp }}(X, Z)_{\geq w}\right)$. It follows that $\operatorname{Ext}^{r}(\mathcal{F}, \mathcal{G})=0$ for all $r \geq 0$. The same argument shows that $\operatorname{Ext}^{r}\left(\left.\mathcal{F}\right|_{V},\left.\mathcal{G}\right|_{V}\right)=0$ for any open subscheme $V$. (Note that any such $V$ must either contain $Z$ or be disjoint from $Z$, since $Z$ is a single $G$-orbit.) Thus, 
$\operatorname{Ext}(\mathcal{F}, \mathcal{G})=0$. This establishes axiom $(2)$ for $Z$ as a subscheme of $X$, and it also establishes axiom (99) for $Z$ itself.

The following result gives the conditions that we will actually use in examples to build $s$-structures.

Theorem 10.2. Suppose $G$ acts on $X$ with finitely many orbits. For each orbit $C \subset X$, let $\mathcal{I}_{C} \subset \mathcal{O}_{X}$ denote the ideal sheaf corresponding to the closed subscheme $i_{C}: \bar{C} \hookrightarrow X$. Suppose each orbit is endowed with an almost $s$-structure, and that the following two conditions hold:

(F1) For each orbit $C,\left.i_{C}^{*} \mathcal{I}_{C}\right|_{C} \in \mathcal{C}_{G}(C)_{\leq 0}$.

(F2) Each $\mathcal{F} \in \mathcal{C}_{G}(C)_{\leq w}$ admits an extension $\mathcal{F}_{1} \in \mathcal{C}_{G}(\bar{C})$ whose restriction to any smaller orbit $C^{\prime} \subset \bar{C}$ is in $\mathcal{C}_{G}\left(C^{\prime}\right)_{\leq w}$.

Then there is a unique s-structure on $X$ whose restriction to each orbit is the given almost s-structure.

Proof. Let $i: Z \hookrightarrow X$ be a reduced closed subscheme. By induction on the number of orbits in $Z$, we will simultaneously prove that $Z$ admits a unique $s$-structure compatible with those on the orbits it contains, and that $Z$ is adhesive as a subscheme of $X$. Let $\mathcal{I}_{Z} \subset \mathcal{O}_{X}$ be its ideal sheaf. If $Z$ consists of a single orbit, then condtion (F1) is equivalent to condition (A1) of Definition [5.1] and then Proposition 10.1 tells us that $Z$ is adhesive. Otherwise, choose an open orbit $C_{0} \subset Z$. Let $\kappa: Y \hookrightarrow Z$ be the complementary reduced closed subscheme. Since $Y$ contains fewer orbits, it has by assumption a unique $s$-structure compatible with those on its orbits, and it is adhesive as a subscheme of $Z$ or $X$. Condition (F2) for $C_{0}$ is none other than the condition (5.2) in the gluing theorem, Theorem 5.3. Invoking that theorem, we find that $Z$ admits a unique $s$-structure compatible with those on $C_{0}$ and on $Y$, and hence with the $s$-structures on the various orbits in $Y$.

Next, because $Y$ is adhesive as a subscheme of $X$, its ideal sheaf $\mathcal{I}_{Y} \subset \mathcal{O}_{X}$ lies in $\mathcal{C}_{G}^{\text {loc }}(X, Y)_{\leq 0}$. The latter category is Serre by Proposition 4.2, and since $\mathcal{I}_{Z} \subset \mathcal{I}_{Y}$, we have $\mathcal{I}_{Z} \in \mathcal{C}_{G}^{\text {loc }}(X, Y)_{\leq 0}$, or $\kappa^{*} i^{*} \mathcal{I}_{Z} \in \mathcal{C}_{G}(Y)_{\leq 0}$. On the other hand, because $C_{0}$ is open in $Z$, we have $\left.\left.i^{*} \mathcal{I}_{Z}\right|_{C_{0}} \simeq i_{C_{0}}^{*} \mathcal{I}_{C_{0}}\right|_{C_{0}} \in \mathcal{C}_{G}\left(C_{0}\right)_{\leq 0}$. It then follows from Theorem 5.3 that $i^{*} \mathcal{I}_{Z} \in \mathcal{C}_{G}(Z)_{\leq 0}$, so condition (A1) holds for $Z$. Next, let $V \subset X$ be the open set obtained by taking the union of $X \backslash Z$ and all orbits that are open in $Z$. Then $V \Subset_{Z} X$, and $V \cap Z$ is a union of orbits that are closed in $V$, so by Proposition 10.1, $V \cap Z$ is adhesive in $V$. Since (A2) is a condition on dense open subschemes, the fact that it holds for the closed subscheme $V \cap Z \subset V$ implies that it holds for $Z \subset X$. Thus, $Z$ is adhesive.

\section{EXAMPLES}

11.1. Trivial $s$-structure. One can always define an $s$-structure on $X$ by declaring all coherent sheaves to be pure of step 0 . In other words,

$$
\mathcal{C}_{G}(X)_{\leq w}=\left\{\begin{array}{ll}
\mathcal{C}_{G}(X) & \text { if } w \geq 0, \\
\{0\} & \text { if } w<0,
\end{array} \quad \mathcal{C}_{G}(X)_{\geq w}= \begin{cases}\mathcal{C}_{G}(X) & \text { if } w \leq 0, \\
\{0\} & \text { if } w>0 .\end{cases}\right.
$$

It is trivial to check that these categories satisfy the axioms for an $s$-structure. Evidently, the altitude of any irreducible closed subscheme $Z \subset X$ is 0 , $\operatorname{sos} \operatorname{scod} Z=$ $\operatorname{cod} Z$. The staggered $t$-structure then reduces to Deligne's perverse coherent $t$ structure as described in B1]. 
11.2. $\mathbb{C}^{\times}$-equivariant sheaves on $\mathbb{C}$. Let $X=\mathbb{C}$, and let $G=\mathbb{C}^{\times}$act on $X$ by multiplication. This action has two orbits: $U=\mathbb{C} \backslash\{0\}$ and $Z=\{0\}$. Let $j: U \hookrightarrow X$ and $i: Z \hookrightarrow X$ be the inclusion maps. On $U$, the $G$-stabilizer of any point is trivial, so any $G$-equivariant sheaf is free. Let us endow $U$ with the trivial $s$-structure.

On $Z$, on the other hand, the category of equivariant coherent sheaves is equivalent to the category of finite-dimensional representations of $G$. Henceforth, we will freely pass back and forth between these categories, regarding representations as sheaves and vice versa. Let $V_{n}$ denote the 1-dimensional representation of $G$ in which each $t \in G$ acts by the formula $t \cdot v=t^{n} v$ for all $v \in V_{n}$. The category of finite-dimensional $G$-representations is semisimple, and we have

$$
V_{n} \otimes V_{m} \simeq V_{n+m} \quad \text { and } \quad \mathcal{H o m}\left(V_{n}, V_{m}\right) \simeq V_{m-n} .
$$

Now, every representation $V$ has a decomposition

$$
V \simeq \bigoplus_{n \in \mathbb{Z}} V_{n} \otimes E_{n}, \quad \text { where } E_{n}=\operatorname{Hom}_{G}\left(V_{n}, V\right),
$$

and in which finitely many $E_{n}$ are nonzero. We define an $s$-structure by

$$
\begin{array}{ll}
V \in \mathcal{C}_{G}(Z)_{\leq w} & \text { if } E_{n}=0 \text { for all } n>w, \\
V \in \mathcal{C}_{G}(Z)_{\geq w} & \text { if } E_{n}=0 \text { for all } n<w .
\end{array}
$$

It is readily seen that $\mathcal{C}_{G}(Z)_{\leq w}$ and $\mathcal{C}_{G}(Z)_{\geq w}$ are both Serre subcategories of $\mathcal{C}_{G}(Z)$. Note also that all higher Ext's vanish in $\mathcal{C}_{G}(Z)$. In view of the isomorphisms (11.1), we see that these categories do indeed define an $s$-structure.

We would now like to invoke Theorem 10.2 to obtain an $s$-structure on all of $X$. Let $A$ be the ring $\mathbb{C}[x]$. We henceforth identify $X$ with $\operatorname{Spec} A$, and we will freely regard $A$-modules as coherent sheaves on $X$. Now, the ideal $I \subset A$ corresponding to $Z$ is the principal ideal $(x)$, and the sheaf $i^{*} I$ can be identified with the vector space $(x) /\left(x^{2}\right)$. An element $t \in G$ acts on $A$ by the formula $t \cdot f(x)=f\left(t^{-1} x\right)$, so we have $i^{*} I \simeq V_{-1}$. Thus, condition (F[1) of Theorem 10.2 is satisfied. Condition (F[2) holds as well: since every object of $\mathcal{C}_{G}(U)$ is free and pure of step 0 , it suffices to observe that $i^{*} A \simeq V_{0} \in \mathcal{C}_{G}(Z)_{\leq 0}$. So that theorem applies, and we get an $s$-structure on $X$.

$A$ itself is a dualizing object in $\mathcal{C}_{G}(X)$. Let us determine the altitudes of $U$ and $Z$ with respect to this choice of dualizing complex. Obviously alt $U=0$. Now, $\omega_{Z} \simeq R i^{!} A$ satisfies

$$
i_{*} \omega_{Z} \simeq R \mathcal{H} \operatorname{Hom}\left(i_{*} \mathcal{O}_{Z}, A\right) \simeq R \mathcal{H o m}(A / I, A) .
$$

There is an obvious free resolution of $A / I$ given by

$$
\cdots \rightarrow 0 \rightarrow x A \rightarrow A \rightarrow 0 \text {. }
$$

$\mathcal{H o m}(x A, A)$ is isomorphic (as an $A$-module with a $G$-action) to $x^{-1} A$. Thus, $R \mathcal{H o m}(A / I, A)$ is represented by the complex

$$
0 \rightarrow A \rightarrow x^{-1} A \rightarrow 0 \rightarrow \cdots .
$$

From this complex, we see that $H^{0}\left(\omega_{Z}\right)=0$, and $H^{1}\left(\omega_{Z}\right) \simeq x^{-1} A / A \simeq V_{1}$. We conclude that $\operatorname{cod} Z=\operatorname{alt} Z=1$.

We therefore have $\operatorname{scod} U=0$ and $\operatorname{scod} Z=2$. Since these differ by 2 , we can invoke the results of Section 9. In particular, the middle perversity, given by

$$
p(U)=0, \quad p(Z)=1
$$


gives rise to a self-dual, finite-length category ${ }^{p} \mathcal{M}(X)$. The simple objects are

$$
\mathcal{I C}\left(X, \mathcal{O}_{U}\right) \simeq \mathcal{O}_{X} \quad \text { and } \quad \mathcal{I C}\left(Z, V_{n}[n-1]\right) \simeq i_{*} V_{n}[n-1]
$$

where $n$ ranges over all integers. The sheaf $x^{-1} A$ is an example of a nonsemisimple object of ${ }^{p} \mathcal{M}(X)$. The following short exact sequence ${ }^{1}$ shows how it is built up out of simple objects:

$$
0 \rightarrow \mathcal{I C}\left(X, \mathcal{O}_{U}\right) \rightarrow x^{-1} A \rightarrow \mathcal{I C}\left(Z, V_{1}\right) \rightarrow 0 .
$$

Remark 11.1. The axioms in Definition [3.7 all involve conditions that are only required to hold over some dense open subscheme. Many statements and arguments in this paper could be simplified if one could assume that those conditions held over the whole scheme (as one can if the scheme is a single $G$-orbit).

However, even in the small example considered here, some of those conditions fail over the whole scheme. For instance, $\mathcal{C}_{G}(X)_{\geq 0}$ is not closed under quotients: the module $x^{2} A$ belongs to $\mathcal{C}_{G}(X)_{\geq 0}$, but its quotient $\left(x^{2}\right) /\left(x^{3}\right) \simeq i_{*} V_{-2}$ does not. Similarly, it is not true that $\operatorname{Ext}^{1}\left(i_{*} V_{-1}, x^{2} A\right)=0$ : one can calculate that $\operatorname{Ri}^{!}\left(x^{2} A\right) \simeq$ $V_{-1}[-1]$ much as we calculated $\omega_{Z}$ above, and then one has $\operatorname{Ext}^{1}\left(i_{*} V_{-1}, x^{2} A\right) \simeq$ $\operatorname{Hom}\left(V_{-1}, R i^{!} x^{2} A[1]\right) \simeq \operatorname{Hom}\left(V_{-1}, V_{-1}\right) \simeq \mathbb{C}$.

11.3. Borel-equivariant sheaves on the flag variety for $S L_{2}(\mathbb{C})$. Now, let $G$ be the group of all matrices of the form $\left(\begin{array}{cc}c & d \\ 0 & c^{-1}\end{array}\right)$ with $c \in \mathbb{C}^{\times}, d \in \mathbb{C}$, and let $G$ act on $X=\mathbb{C P}^{1}$ by the formula

$$
\left(\begin{array}{cc}
c & d \\
0 & c^{-1}
\end{array}\right) \cdot(a: b)=\left(c a+d b: c^{-1} b\right) .
$$

$G$ is, of course, a Borel subgroup of $S L_{2}(\mathbb{C})$, and $\mathbb{C P}^{1}$ can be identified with the flag variety of $S L_{2}(\mathbb{C})$. Under this identification, the action described above coincides with the obvious action of a Borel subgroup on a flag manifold. There are two orbits: the singleton $Z=\{(1: 0)\}$, and the open subscheme $U=\{(a: b) \mid a \neq 0\}$.

Let $A$ denote the ring $\mathbb{C}[x, y]$. Let us make $A$ into a graded ring by declaring both $x$ and $y$ to be homogeneous elements of degree 1 . Then we may identify $X$ with $\operatorname{Proj} A$. $G$ acts on $A$ as follows:

$$
\left(\begin{array}{cc}
c & d \\
0 & c^{-1}
\end{array}\right) \cdot x=c^{-1} x-d y \quad \text { and } \quad\left(\begin{array}{cc}
c & d \\
0 & c^{-1}
\end{array}\right) \cdot y=c y
$$

Let $I$ denote the principal homogeneous ideal $(y) \subset A$. This is a $G$-stable ideal, corresponding to the closed subscheme $Z$.

The $G$-stabilizer of the point $(0: 1) \in U$ is the subgroup $T \subset G$ of diagonal matrices $\left(\begin{array}{cc}c & 0 \\ 0 & c^{-1}\end{array}\right)$, so the category $\mathcal{C}_{G}(U)$ is equivalent to the category of finitedimensional $T$-representations. Let $V_{n}$ denote the 1-dimensional $T$-representation in which elements of $T$ act on $v \in V_{n}$ by

$$
\left(\begin{array}{cc}
c & 0 \\
0 & c^{-1}
\end{array}\right) \cdot v=c^{n} v
$$

We define an $s$-structure on $U$ in the same way we did in the previous section for the closed orbit of $\mathbb{C}^{\times}$-action on $\mathbb{C}$ : a sheaf $\mathcal{F} \in \mathcal{C}_{G}(U)$ belongs to $\mathcal{C}_{G}(U)_{\leq w}$

\footnotetext{
${ }^{1}$ This particular short exact sequence was suggested to me by E. Vasserot as a desideratum for a well-behaved $t$-structure on $\mathcal{D}_{\mathbb{C}^{\times}}^{\mathrm{b}}(\mathbb{C})$. This entire paper was essentially written in an attempt to give meaning to this sequence.
} 
(resp. $\mathcal{C}_{G}(U)_{\geq w}$ ) if the corresponding $T$-representation contains no summand $V_{n}$ with $n>w$ (resp. $n<w)$.

Consider the element $t=x / y$ in the fraction field of $A$. This element has degree 0 , and we can identify $U$ with Spec $\mathbb{C}[t]$. The $G$-action on $\mathbb{C}[t]$ is given by

$$
\left(\begin{array}{cc}
c & d \\
0 & c^{-1}
\end{array}\right) \cdot t=\frac{c^{-1} x-d y}{c y}=c^{-2} t-d / c .
$$

The ideal $J$ corresponding to the point $(0: 1)$ is the principal ideal $(t)$. (Note that this ideal is $T$-stable.) Given a $\mathbb{C}[t]$-module $M$ with a $G$-action (or in other words, a sheaf in $\left.\mathcal{C}_{G}(U)\right)$, the corresponding $T$-representation is given by $M / J M$.

Now, consider the twisted module $\mathcal{O}_{X}(n)=A(n)$. The restriction of this sheaf to $U$ can be identified with a free $\mathbb{C}[t]$-module $M_{n}$ generated by $y^{n}$. The $T$-action on this module is given by

$$
\left(\begin{array}{cc}
c & 0 \\
0 & c^{-1}
\end{array}\right) \cdot y^{n} t^{k}=(c y)^{n}\left(c^{-2} t\right)^{k}=c^{n-2 k} y^{n} t^{k}
$$

for any $k \geq 0$. In particular, we see that $M_{n} / J M_{n} \simeq V_{n}$. Thus, $\left.\mathcal{O}_{X}(n)\right|_{U}$ is pure of step $n$. Moreover, since every $T$-representation is a direct sum of various $V_{n}$ 's, we see that every sheaf in $\mathcal{C}_{G}(U)$ is isomorphic to a direct sum of various $\left.\mathcal{O}_{X}(n)\right|_{U}$ 's.

We now turn our attention to $Z$. The category $\mathcal{C}_{G}(Z)$ is, of course, equivalent to the category of finite-dimensional $G$-representations. We define an $s$-structure on $\mathcal{C}_{G}(Z)$ as follows: given a $G$-representation $V$, we regard it as a $T$-representation, and then we declare that $V \in \mathcal{C}_{G}(Z)_{\leq w}$ if $V$ contains no summand $V_{n}$ with $n<-w$. Note that this is opposite to the convention used on $U$. Similarly, we declare that $V \in \mathcal{C}_{G}(Z)_{\geq w}$ if $V$ contains no summand $V_{n}$ with $n>-w$. As a $T$-representation, $i^{*} I \simeq(y) /\left(y^{2}\right)$ is isomorphic to $V_{1}$, so $i^{*} I \in \mathcal{C}_{G}(Z)_{\leq-1}$. Thus, condition (FI) of Theorem 10.2 holds.

Next, we check condition (F2). Since every sheaf in $\mathcal{C}_{G}(U)$ is a direct sum of various $\left.\mathcal{O}_{X}(n)\right|_{U}$ 's, it suffices to show that $i^{*} \mathcal{O}_{X}(n) \in \mathcal{C}_{G}(Z)_{\leq n}$. The submodule of $A / I$ consisting of all homogeneous elements of degree $n$ can be identified with the vector space

$$
\mathbb{C}\left\{x^{n}, x^{n-1} y, \cdots, y^{n}\right\} / \mathbb{C}\left\{x^{n-1} y, x^{n-2} y^{2}, \cdots, y^{n}\right\} .
$$

This is clearly isomorphic to $V_{-n}$ as a $T$-representation, so we conclude that $i^{*} \mathcal{O}_{X}(n) \in \mathcal{C}_{G}(Z)_{<n}$, as desired. Theorem 10.2 now gives us an $s$-structure on all of $X$.

Finally, let us compute the altitudes of $U$ and $Z$. We take $A$ itself as the dualizing complex. Evidently, we have alt $U=0$. A calculation very similar to that carried out in the preceding section shows that $H^{0}\left(\omega_{Z}\right)=0$ and $H^{1}\left(\omega_{Z}\right) \simeq y A / A \simeq V_{-1}$. We conclude that alt $Z=1$.

It can be checked that $\operatorname{scod} U=0$ and $\operatorname{scod} Z=3$, so from the results of Section 9, we find that the staggered $t$-structure on $\mathcal{D}_{G}^{\mathrm{b}}(X)$ with respect to the perversity $p(U)=0, p(Z)=1$ has a finite-length heart. The simple objects are

$$
\mathcal{I C}\left(X,\left.\mathcal{O}_{X}(n)\right|_{U}\right) \quad \text { and } \quad \mathcal{I C}\left(Z, V_{n}[-n-1]\right),
$$

where $n$ ranges over all integers.

\section{REFERENCES}

[A1] P. Achar, On the equivariant $K$-theory of the nilpotent cone for the general linear group, Represent. Theory 8 (2004), 180-211. 
[A2] P. Achar, On the quasi-hereditary property for staggered sheaves, Trans. Amer. Math. Soc., to appear.

[AS1] P. Achar and D. Sage, Perverse coherent sheaves and the geometry of special pieces in the unipotent variety, Adv. Math. 220 (2009), 1265-1296.

[AS2] P. Achar and D. Sage, Staggered sheaves on flag varieties, C. R. Math. Acad. Sci. Paris, Ser. I 347 (2009), 139-142.

[AT1] P. Achar and D. Treumann, Baric structures on triangulated categories and coherent sheaves, arXiv:0808.3209

[AT2] P. Achar and D. Treumann, Purity and decomposition theorems for staggered sheaves, arXiv:0808.3210

[BBD] A. Bellinson, J. Bernstein, and P. Deligne, Faisceaux pervers, Analyse et topologie sur les espaces singuliers, I (Luminy, 1981), Astérisque, vol. 100, Soc. Math. France, Paris, 1982, pp. $5-171$.

[B1] R. Bezrukvnikov, Perverse coherent sheaves (after Deligne), arXiv:math.AG/0005152

[B2] R. Bezrukavnikov, Quasi-exceptional sets and equivariant coherent sheaves on the nilpotent cone, Represent. Theory 7 (2003), 1-18.

[B3] R. Bezrukavnikov, Cohomology of tilting modules over quantum groups and t-structures on derived categories of coherent sheaves, Invent. Math 166 (2006), 327-357.

$[\mathrm{H}] \quad$ R. Hartshorne, Residues and duality, Lecture Notes in Mathematics, no. 20, SpringerVerlag, Berlin, 1966.

[T] D. Treumann, Staggered $t$-structures on toric varieties, arXiv:0806.0696.

Department of Mathematics, Louisiana State University, Baton Rouge, LA 70803

E-mail address: pramod@math.lsu.edu 